\title{
Dual polar spaces of arbitrary rank
}

\author{
Simon Huggenberger* \\ (Communicated by A. Cohen)
}

\begin{abstract}
In 1982 P. Cameron gave a characterisation of dual polar spaces of finite rank viewed as point-line spaces. This characterisation makes essential use of the fact that dual polar spaces of finite rank have finite diameter. Our goal is to give a characterisation which includes dual polar spaces of infinite rank. Since dual polar spaces of infinite rank are disconnected, we introduce a point-relation that denotes pairs of points at "maximal distance", and we call this an opposition relation. This approach is in the spirit of the theory of twin buildings.
\end{abstract}

\section{Introduction}

P. Cameron gave in [4] five axioms to characterise dual polar spaces of finite rank. In his axioms he uses the fact that these spaces are connected. In contrast, the dual of a polar space of infinite rank is not connected as we will see in the end of Section 2. Thus, there is no straightforward way to generalise the axioms of $\mathrm{P}$. Cameron to obtain a characterisation of dual polar spaces of arbitrary rank.

In order to relate objects belonging to distinct connected components of a point-line space, we introduce a point-relation which we call an opposition relation. The pairs of this relation can be viewed as point-pairs that have maximal distance. Hence in a dual polar space of finite rank $r$, a suitable opposition relation is the set of all pairs of points that have distance $r$ which is actually the diameter of this space. By means of such an opposition relation we define a certain class of point-line spaces which we call local dual polar spaces, see Definition 3.4.

In Section 4 we show that every local dual polar space of finite diameter is a genuine dual polar space. In Section 5 we study the examples of arbitrary diameter. We show that these can be reduced to a certain subclass of spaces that consists of at most two connected components. We call the members of this subclass atomic local dual polar spaces. Furthermore, we explain how to obtain polar spaces out of these point-line spaces. We conclude that section by showing that atomic local dual polar spaces are subspaces of dual polar spaces.

*The research of the author is supported by UGent GOA2006, project number 010, "Incidence Geometry". 
In Section 6 we introduce a condition for polar spaces under which their dual polar spaces are local dual polar spaces. It is not known whether there exist dual polar spaces that do not satisfy this condition. Finally, in Section 7 we give a one-to-one correspondence between equivalence classes of polar spaces satisfying the condition given in Section 6 and equivalence classes of atomic local dual polar spaces.

The axioms of P. Cameron that characterise dual polar spaces of finite rank (more precisely, of rank $n$ ) are the following:

(A1) Given any point $x$ and line $L$, there is a unique point of $L$ nearest to $x$.

(A2) $G$ is connected and has diameter $n$.

(A3) If $x$ and $y$ are points with $\mathrm{d}(x, y)=2$, and $\Delta(x, y)$ is the smallest set of points containing $x$ and $y$ and containing any point collinear with two of its points, then $\Delta(x, y)$ has diameter 2 .

(A4) For $d \leq n$, let $x, y, z$ be points with $\mathrm{d}(x, y)=2, \mathrm{~d}(x, z)=\mathrm{d}(y, z)=d$. Then

(a) there is a point $w$ joined to $x$ and $y$ with $\mathrm{d}(z, w)=d-1$; or

(b) there is a point $w \in \Delta(x, y)$ with $\mathrm{d}(z, w)=d-2$.

(A5) For $d \leq n$, let $x, y, z$ be points with $\mathrm{d}(x, y)=d, \mathrm{~d}(x, z)=d-1, \mathrm{~d}(y, z)=1$.

Then there is a point $w$ with $\mathrm{d}(x, w)=1, \mathrm{~d}(y, w)=d-1, \mathrm{~d}(z, w)=d$.

The characterisation we give in Definition 3.4 can be seen as a generalisation of Axiom (A5). In fact, it is much stronger since it is stated for any convex subspace with finite diameter where (A5) only refers to lines. Additionally, Definition 3.4 asks implicitly a generalisation of the Axioms (A1) and (A4). Axiom (A3) is an immediate consequence of Lemma 3.10. Propositions 3.13 and 3.16 provide an explicit generalisation of (A1) and (A4). Finally, the reduction to atomic dual polar spaces, see Definition 5.3, can be seen as the counterpart to (A2).

\section{Preliminaries}

The notation, terms and definitions used in this article are predominantly adopted from [5], as long as this was possible.

A point-line space $\mathscr{S}=(\mathcal{P}, \mathcal{L})$ is a pair consisting of a set $\mathcal{P}$, whose elements are called points and a set $\mathcal{L} \subset \mathfrak{P}(\mathcal{P})$ of subsets of $\mathcal{P}$ with cardinality at least 2 , which are called lines. If all points are subsets of a common set, we sometimes regard a line as the union of its points. A partially linear space is a point-line space such that no two different lines have two different points in common. Sometimes we treat $\mathscr{S}$ as its own point set, so we might write $x \in \mathscr{S}$ instead of $x \in \mathcal{P}$. A subspace of $\mathscr{S}$ is a point-line space $\mathscr{S}^{\prime}=\left(\mathcal{P}^{\prime}, \mathcal{L}^{\prime}\right)$ with $\mathcal{P}^{\prime} \subseteq \mathcal{P}$ and $\mathcal{L}^{\prime} \subseteq \mathcal{L}$ such that every line in $\mathcal{L} \backslash \mathcal{L}^{\prime}$ has at most one point with $\mathcal{P}^{\prime}$ in common and every line in $\mathcal{L}^{\prime}$ is contained in $\mathcal{P}^{\prime}$. We write $\mathscr{S}^{\prime} \leq \mathscr{S}$, and $\mathscr{S}^{\prime}<\mathscr{S}$ if $\mathscr{S}^{\prime}$ is properly contained. Since $\mathscr{S}^{\prime}$ is determined by its point set, we call $\mathcal{P}^{\prime}$ itself a subspace. Obviously a subspace of a partially linear space is again partially linear. A proper subspace which intersects every line is called a hyperplane. For a set of points $M$, we denote by $\langle M\rangle$ the smallest subspace which contains $M$, called the span of $M$. For a family of points $p_{0}, \ldots p_{s}$ and a family of sets of points $M_{0}, \ldots, M_{r}$ we will write $\left\langle p_{0}, \ldots, p_{s}, M_{0}, \ldots, M_{r}\right\rangle$ rather than $\left\langle\left\{p_{0}, \ldots, p_{s}\right\} \cup M_{0} \cup \cdots \cup M_{r}\right\rangle$. 
Lemma 1.1. Let $M$ be a set of points of a partially linear space. Then we have $\langle M\rangle=$ $\bigcup_{X \in \mathfrak{P}_{e}(M)}\langle X\rangle$, where $\mathfrak{P}_{e}(M)$ is the set of all finite subsets of $M$.

Proof. If $M$ is finite, this is obvious. Now let $M$ be infinite. Further let $X$ and $Y$ be two finite subsets of $M$. All points on a line joining a point in $\langle X\rangle$ with one in $\langle Y\rangle$ are contained in $\langle X \cup Y\rangle$. Hence $\bigcup_{X \in \mathfrak{P}_{e}(M)}\langle X\rangle$ is a subspace which contains $M$. Since $\langle M\rangle \geq\langle X\rangle$ for all $X \in \mathfrak{P}_{e}(M)$, equality holds.

Points that are contained in a common line are called collinear. For two collinear points $x$ and $y$, we write $x \sim y$. In a partially linear space we denote the line joining $x$ and $y$ by $x y$ if $x \neq y$. The set of all points being collinear to a point $x$ is denoted by $x^{\sim}$. For a set of points $M$, the set of points collinear to every point of $M$ is denoted by $M^{\sim}:=\bigcap_{p \in M} p^{\sim}$. Frequently used properties in this context are stated in the following lemma.

Lemma 1.2. Let $M$ and $N$ be sets of points of a point-line space. If $N \subseteq M$, then $N^{\sim} \supseteq M^{\sim}$. Furthermore, $M \subseteq M^{\sim \sim}$ and $M^{\sim}=M^{\sim \sim \sim}$.

Proof. With $N \subseteq M$ we obtain $M^{\sim}=\bigcap_{p \in M} p^{\sim}=\left(\bigcap_{p \in N} p^{\sim}\right) \cap\left(\bigcap_{p \in M \backslash N} p^{\sim}\right) \subseteq N^{\sim}$. Since every point of $M$ is collinear to every point of $M^{\sim}$, we obtain $M \subseteq M^{\sim \sim}$. This implies $M^{\sim} \subseteq\left(M^{\sim}\right)^{\sim \sim}$ and $M^{\sim} \supseteq\left(M^{\sim \sim}\right)^{\sim}$.

A point-line space is called singular if every two points are collinear. A point-line space consisting of one single point is called a singleton.

Recall that a chain is a totally ordered set. A well-ordered chain is a chain such that every subchain has a minimal element. Whenever we talk about a chain of subspaces, the used order is inclusion, if no other order is mentioned.

The rank of a singular subspace $\mathscr{S}$ is denoted by $\operatorname{rk}(\mathscr{S})$ and equals $\alpha-2$, where $\alpha$ is the supremum over the lengths of well-ordered chains of subspaces of $\mathscr{S}$. Hence the rank of the empty space is -1 and the rank of a singleton is 0 . Note that there might exist chains of cardinality less than $\alpha$ that are maximal. For a point-line space $\mathscr{S}$ let $\mathfrak{S}(\mathscr{S}):=\left\{X \leq \mathscr{S} \mid X \subseteq X^{\sim}\right\}$ denote the set of all singular subspaces of $\mathscr{S}$. The singular rank of $\mathscr{S}$ is defined as $\operatorname{srk}(\mathscr{S}):=\sup \{\operatorname{rk}(X) \mid X \in \mathfrak{S}(\mathscr{S})\}$.

Let $\mathscr{S}=(\mathcal{P}, \mathcal{L})$ be a point-line space and $x$ and $y$ be two points of $\mathscr{S}$. With $\mathrm{d}(x, y)$ we denote the distance between $x$ and $y$ in the collinearity graph, i.e. the graph induced by $\sim$ on the vertex set $\mathcal{P}$. Hence, $\mathrm{d}(x, y)=0$ only occurs for $x=y$ and $\mathrm{d}(x, y)=n$ means that $y$ is collinear to a point at distance $n-1$ to $x$. Beside the distance we also adopt the term path from the collinearity graph. Recall that a path is a finite sequence of points $\left(x_{i}\right)_{0 \leq i<n}$, where $n \in \mathbb{N}$, such that $\left\{x_{i}, x_{i+1}\right\}$ forms an edge for all $i<n-1$. Two points are called connected if there is a path containing them. A path of minimal length between two connected points is called a geodesic. For two points $x$ and $y$ that are not connected, we set $\mathrm{d}(x, y)=\infty$. For two sets of points $X$ and $Y$ we define $\mathrm{d}(X, Y):=\min \{\mathrm{d}(x, y) \mid x \in X \wedge y \in Y\}$ and write $\mathrm{d}(x, X)$ rather than $\mathrm{d}(\{x\}, X)$. With $\operatorname{diam}(M):=\sup \left\{-1, \mathrm{~d}(x, y) \mid(x, y) \in M^{2}\right\}$ we denote the diameter of a set of points $M$. 
A subspace of a point-line space is called convex if it contains for every pair of points all geodesics. Let $\mathfrak{G}$ be the set of all convex subspaces of $\mathscr{S}$. For a subset $X \subseteq \mathscr{S}$, we call $\langle X\rangle_{\mathrm{g}}:=\bigcap\{\mathcal{C} \mid X \subseteq \mathcal{C} \in \mathfrak{G}\}$ the convex span of $X$. This intersection is well defined since $\mathscr{S} \in \mathfrak{G}$. For points $x_{0}, \ldots, x_{n}$ and a set of points $X$, we write $\left\langle x_{0}, \ldots, x_{n}, X\right\rangle_{\mathrm{g}}$ rather than $\left\langle\left\{x_{0}, \ldots, x_{n}\right\} \cup X\right\rangle_{\mathrm{g}}$.

A morphism $\varphi:\left(\mathcal{P}_{0}, \mathcal{L}_{0}\right) \rightarrow\left(\mathcal{P}_{1}, \mathcal{L}_{1}\right)$ of point-line spaces is a map from $\mathcal{P}_{0}$ to $\mathcal{P}_{1}$ such that the image of every line in $\mathcal{L}_{0}$ is contained in some line of $\mathcal{L}_{1}$. An isomorphism is a bijective morphism $\varphi$ such that the inverse map $\varphi^{-1}$ is again a morphism.

\section{Polar spaces and dual polar spaces}

In this section, we will prove some basic facts about polar spaces and dual polar spaces of arbitrary rank. Some of these facts are easy consequences of deeper results in the finite rank case. Although some of the generalisations to the arbitrary case do not cause any difficulties, we give the proofs since we do not know suitable references in the literature.

In polar spaces it is common to denote collinearity by the symbol $\perp$. Correspondingly, one writes $x^{\perp}$ instead of $x^{\sim}$. We will use this notation, too, when we talk about polar spaces and will keep the notation with $\sim$ for other point-line spaces. By now, this might seem confusing, but the advantage of doing so will become clear later.

A polar space $\mathscr{S}=(\mathcal{P}, \mathcal{L})$ is a point-line space such that for every point $p$ the set $p^{\perp}$ is a hyperplane or the whole point set, see [5]. An obviously equivalent definition by F. Buekenhout and E. Shult is the following:

(BS) Let $(p, l) \in \mathcal{P} \times \mathcal{L}$. Then $p$ is collinear to either all or exactly one point of $l$.

We mention both definitions since each has its advantages in certain situations.

Let $\mathscr{S}=(\mathcal{P}, \mathcal{L})$ be a polar space. The radical $\operatorname{Rad}(\mathscr{S}):=\left\{p \in \mathcal{P} \mid p^{\perp}=\mathcal{P}\right\}$ is a subspace of $\mathscr{S}$ that consists of all points which are collinear to all others. A polar space is called non-degenerate if the radical is empty. A maximal singular subspace of a polar space is called a generator. The rank of a polar space is the supremum $\alpha$ over the lengths of well-ordered chains of singular subspaces all containing $\operatorname{Rad}(\mathscr{S})$ properly.

We start with some useful properties of polar spaces.

Lemma 2.1. Let $M$ be a set of points of a polar space. Then $\left\langle M^{\perp}\right\rangle=M^{\perp}=\langle M\rangle^{\perp}$.

Proof. [2, Lemma 7.4.7(iii)].

Let $M$ be a set of points of a point-line space. The points of $M$ are mutually collinear if and only if $M \subseteq M^{\perp}$. Hence, a subspace $U$ is singular if and only if $U \subseteq U^{\perp}$.

Lemma 2.2. Let $M$ be a set of mutually collinear points of a polar space. Then the subspace $\langle M\rangle$ is singular.

Proof. [2, Lemma 7.4.7(v)]. 
Using Zorn's Lemma one sees that every polar space has generators and that every singular subspace of a polar space is contained in some generator. Furthermore, with the lemma above, every set of mutually collinear points is contained in a generator.

In a polar space $\mathscr{S}$, two generators $M$ and $N$ are called adjacent when they intersect in a common hyperplane, denoted by $M \sim N$. Let $\mathcal{G}$ be the set of generators. The graph on $\mathcal{G}$ induced by $\sim$ is called the dual polar graph. Let $\mathcal{C}$ be the set of maximal cliques, i.e. maximal complete subgraphs, of the dual polar graph. Then $(\mathcal{G}, \mathcal{C})$ is a point-line space, called dual polar space. Point-line spaces which are isomorphic to such a space are also called dual polar spaces. Since for two generators $M$ and $N$ the term $\mathrm{d}(M, N)$ is already occupied for the shortest distance of a point of $M$ to a point of $N$, we denote the distance of two generators in the dual polar graph by $\mathrm{d}^{*}(M, N)$.

In a non-degenerate polar space of finite rank $n$, all lines of which have cardinality at least 3 , every singular subspace is contained in some singular subspace of rank $n-1$; see [7]. The equivalence between the axioms used there and the ones used here is shown in [3]. So in this case, a singular subspace $L$ of rank $n-2$ gives rise in a natural way to a line of the dual, namely the set of maximal singular subspaces containing $L$. In terms of shadow spaces, see [2], the dual is just the $\{n-1\}$-space, if $n \in \mathbb{N}$ is the rank of $\mathscr{S}$.

In polar spaces of arbitrary rank it may occur that there are generators of different $\operatorname{rank}^{1}$; see [6] or Section 8 for an example. But there are some weaker conditions which still hold.

Lemma 2.3. Let $\mathscr{S}$ be a polar space and let $M$ and $N$ be two generators of $\mathscr{S}$. Then $M \cap N$ is a hyperplane of $M$ if and only if $M \cap N$ is a hyperplane of $N$.

Proof. Assume that $H:=M \cap N$ is a proper hyperplane of $M$ and let $l$ be a line of $N$. Take a point $p \in M \backslash H$. Then by (BS) there is a point $q$ on $l$ which is collinear to $p$. Since $q^{\perp} \cap M$ contains $H$ and $p$, we obtain $M \leq q^{\perp}$. This implies that $\langle q, M\rangle$ is singular and hence $q \in M$ by the maximality of $M$. Thus, $q \in H$ and $H$ intersects $l$.

Proposition 2.4. Let $X, Y$ and $Z$ be generators of a polar space, which are pairwise adjacent. Then $X, Y$ and $Z$ have a hyperplane in common.

Proof. We may assume that $X, Y$ and $Z$ are pairwise distinct, since otherwise we are done. Since $X$ and $Y$ are adjacent, they have a hyperplane $H$ in common. If $H \leq Z$ we are done by Lemma 2.3.

Now assume $H \not Z Z$. Suppose $Z \cap X \leq H$. Then for a point $p \in X \backslash H$ and a point $p \in H$, we obtain $p q \cap H=\{q\}$. Since $Z$ intersects $X$ in a hyperplane, this implies $q \in Z$ and hence, $H \leq Z$, a contradiction. Thus, there is a point $x \in Z \cap X \backslash H$ and, analogously, a point $y \in Z \cap Y \backslash H$. Since $x$ and $y$ are contained in $Z$, they are collinear. Hence, $x$ and $H$ are contained in $y^{\perp}$ and therefore $X \leq y^{\perp}$. Thus, $\langle y, X\rangle$ is singular, a contradiction to $y \notin X$ and the maximality of $X$.

From this proposition follows that every line of a dual polar space corresponds to a hyperplane of a generator of the underlying polar space. Conversely, hyperplanes of

\footnotetext{
${ }^{1}$ In this case, of course, all generators have infinite rank.
} 
generators which are contained in two different generators correspond to lines of the dual polar space. Note that there might be hyperplanes of generators which are contained in only one generator and therefore do not correspond to any line of the dual.

There are non-isomorphic polar spaces whose duals are isomorphic. To study dual polar spaces it suffices to check only one representative of each class of polar spaces with isomorphic duals. In every such class there is one polar space with rather nice properties.

Let $\mathscr{S}=(\mathcal{P}, \mathcal{L})$ be a polar space. For every point $p \in \mathcal{P}$ we set $p^{\rho}:=\langle p, \operatorname{Rad}(\mathscr{S})\rangle$ and for every line $l \in \mathcal{L}$ we set $l^{\rho}:=\left\{p^{\rho} \mid p \in l\right\}$. Finally we set $\mathcal{P}^{\rho}:=\left\{p^{\rho} \mid p \in\right.$ $\mathcal{P} \backslash \operatorname{Rad}(\mathscr{S})\}$ and $\mathcal{L}^{\rho}:=\left\{l^{\rho} \mid l \in \mathcal{L} \wedge l \cap \operatorname{Rad}(\mathscr{S})=\emptyset\right\}$. Then $\mathscr{S}^{\rho}:=\left(\mathcal{P}^{\rho}, \mathcal{L}^{\rho}\right)$ is again a polar space that is non-degenerate and therefore called the associated non-degenerate polar space; see $[5,2.4]$ and $[6,3.1]$.

Proposition 2.5. Let $\mathscr{S}$ be a polar space. Then the dual polar spaces of $\mathscr{S}$ and $\mathscr{S}^{\rho}$ are isomorphic.

Proof. For a subspace $U \leq \mathscr{S}$, we set $U^{\rho}:=\left\{p^{\rho} \mid p \in U \backslash \operatorname{Rad}(\mathscr{S})\right\}$. By mapping every subspace $U$ to $U^{\rho}$ we obtain a bijection between the set of subspaces of $\mathscr{S}$ that contain $\operatorname{Rad}(\mathscr{S})$ and the set of subspaces of $\mathscr{S}^{\rho}$. Thereby singular subspaces are mapped on singular subspaces, non-singular are mapped on non-singular ones and proper inclusion is preserved. Hence, the ranks of $\mathscr{S}$ and $\mathscr{S}^{\rho}$ coincide and the given bijection induces a bijection between the generators of $\mathscr{S}$ and those of $\mathscr{S}^{\rho}$. This bijection preserves the adjacency of generators and the claim follows.

We do not know whether there are non-isomorphic non-degenerate polar spaces with isomorphic duals. However, as we will see in Section 7, we can count such a situation out whenever the polar spaces admit a natural opposition relation.

Motivated by the previous proposition we may constrain our focus on non-degenerate polar spaces. The main advantage of doing so is based on the following property:

\section{Proposition 2.6. Every non-degenerate polar space is partially linear.}

Proof. See [6, Proposition 3.1(vii)].

A partially linear space that is singular is called linear. In other words, in a linear space, every pair of distinct points is joined by exactly one line and every line is a subspace. Generators of non-degenerate polar spaces are more than just linear. For this we now introduce a certain class of linear spaces. A possibly degenerate projective space is a linear space satisfying the following property of Veblen and Young:

(VY) For every pair $(l, k)$ of disjoint lines and every point $p \in \mathcal{P} \backslash(l \cup k)$ there is at most one line through $p$ meeting both $l$ and $k$.

A projective space is called degenerate if it contains short lines, i.e. lines of cardinality 2. Usually, projective spaces are required to be non-degenerate. However, if we talk about projective spaces, we always allow them to be degenerate. 
Proposition 2.7. Every singular subspace of a non-degenerate polar space is a projective space.

Proof. See [6, Theorem 3.2].

A set of points $X$ is called independent if $p \notin\langle X \backslash\{p\}\rangle$ for every $p \in X$. A set of points which is not independent will be called dependent. An independent set of points $B$ of a point-line space $\mathscr{S}$ with $\langle B\rangle=\mathscr{S}$ is called a basis of $\mathscr{S}$.

In projective spaces, the union of two disjoint independent sets of points $X$ and $Y$ is again independent if and only if $\langle X\rangle \cap\langle Y\rangle=\emptyset$. Hence, using Zorn's Lemma, every independent set of points is contained in a basis. Every two bases of a projective space have the same cardinality (see [1, II, §7, no.2, Theorem 3] for a proof), namely the rank +1 . Furthermore, $|B \backslash(B \cap C)|=|C \backslash(B \cap C)|$ holds for two bases $B$ and $C$. Motivated by this we define the corank of a subspace $U$ in a projective space $V$ as $\operatorname{crk}_{V}(U):=\left|B \backslash B_{U}\right|$, where $B_{U}$ is a basis of $U$ and $B$ is a basis of $V$ containing $B_{U}$.

Remark 2.8. The fact that a projective space $S$ has a basis implies that every maximal well-ordered chain of subspaces of $S$ has length $\operatorname{rk}(S)+2$.

Let $\mathscr{S}$ be a polar space. Since there is a canonical bijection between singular subspaces of $\mathscr{S}$ containing $\operatorname{Rad}(\mathscr{S})$ and the singular subspaces of $\mathscr{S}^{\rho}$, we conclude that a maximal well-ordered chain of subspaces of a given generator $G \leq \mathscr{S}$ all containing $\operatorname{Rad}(\mathscr{S})$ properly has length $\operatorname{crk}_{G}(\operatorname{Rad}(\mathscr{S}))$.

Definition 2.9. Let $\mathscr{S}$ be a polar space. Further let $U \subseteq \mathscr{S}$ be a set of points and $V \leq \mathscr{S}$ a subspace of $\mathscr{S}$. Then we set $U \oplus V:=\left\langle U, U^{\perp} \cap V\right\rangle$.

For a single point $p$ we will write $p \oplus V$ rather than $\{p\} \oplus V$. If $M$ is a generator then $p^{\perp}$ contains a hyperplane of $M$ and hence $p \oplus M$ is again a generator by Lemma 2.3. Moreover, if $p \notin M$ then $p \oplus M$ is the unique generator adjacent to $M$ and containing $p$.

We call two singular subspaces $M$ and $N$ of a point-line space commensurate if $\operatorname{crk}_{M}(M \cap N)=\operatorname{crk}_{N}(M \cap N) \in \mathbb{N}$.

Proposition 2.10. Let $M$ and $N$ be two generators of a polar space. Then either $M$ and $N$ are commensurate and $\mathrm{d}^{*}(M, N)=\operatorname{crk}_{M}(M \cap N)$ or $\mathrm{d}^{*}(M, N), \operatorname{crk}_{M}(M \cap N)$ and $\operatorname{crk}_{N}(M \cap N)$ are all infinite.

Proof. Set $H:=M \cap N$. First let $\operatorname{crk}_{M}(H)=: r<\infty$. We prove $\mathrm{d}^{*}(M, N) \leq r$ by induction. If $r=0$, then $M=N$ and therefore $\mathrm{d}^{*}(M, N)=0$. For $r>0$ let $\left\{b_{i} \mid 0 \leq i<r\right\}$ be a set of points such that $\left\langle H, b_{i} \mid 0 \leq i<r\right\rangle=M$. Set $N_{0}:=N$ and $N_{i+1}:=b_{i} \oplus N_{i}$ for $0 \leq i<r$. Then $N_{i} \sim N_{i+1}$ and $N_{r}=M$. Thus, we conclude $\mathrm{d}^{*}(M, N) \leq r$.

Now let $\mathrm{d}^{*}(M, N)=: d<\infty$. Then there are generators $N_{i}$ for $0 \leq i \leq d$ such that $N_{0}=N, N_{d}=M$ and $N_{i} \sim N_{i+1}$ for $i<d$. Since $\operatorname{crk}_{N_{i}}\left(N_{i} \cap N_{i-1}\right)=1$ for $i>0$, we obtain $\operatorname{crk}_{N_{i}}\left(N_{i} \cap N_{0}\right) \leq i$ and hence $\operatorname{crk}_{M}(H) \leq d$. 
Remark 2.11. There are polar spaces possessing generators $M$ and $N$ such that $\operatorname{rk}(M)>$ $\operatorname{rk}(N)$; see Section 8. In such a case we always obtain $\operatorname{crk}_{M}(M \cap N)>\operatorname{crk}_{N}(M \cap N)$. Moreover, $\operatorname{rk}(M)=\operatorname{crk}_{M}(M \cap N) \operatorname{since} \operatorname{rk}(M \cap N) \leq \operatorname{rk}(N)<\operatorname{rk}(M)$.

Lemma 2.12. Let $U$ be a singular subspace of a polar space $\mathscr{S}$ with $\operatorname{rk}(U)<\infty$ and let $M \leq \mathscr{S}$ be a generator. Then

(i) $\operatorname{rk}(U)=\operatorname{crk}_{M}\left(M \cap U^{\perp}\right)+\operatorname{rk}(M \cap U)$ and

(ii) $U \oplus M$ is a generator with $\mathrm{d}^{*}(M, U \oplus M)=\operatorname{crk}_{U}(U \cap M)$.

Proof. Set $k:=\operatorname{rk}(M \cap U)$ and $n:=\operatorname{rk}(U)$. Let $\left(p_{i}\right)_{0 \leq i \leq n}$ be a basis of $U$ such that $\left(p_{i}\right)_{0 \leq i \leq k}$ is a basis of $M \cap U$. Set $M_{0}:=M$ and $M_{i+1}:=p_{k+i+1} \oplus M_{i}$ for $i<n-k$. Then $\left(M_{i}\right)_{0 \leq i \leq n-k}$ is a sequence of pairwise adjacent generators and hence $\mathrm{d}^{*}\left(M, M_{n-k}\right) \leq n-k$.

We know $\left\langle p_{j} \mid j \leq k\right\rangle \leq M_{0}$. Hence we obtain $\left\langle p_{j} \mid j \leq k+i+1\right\rangle \leq M_{i+1}$ since $\left\langle p_{j} \mid j \leq k+i\right\rangle \leq p_{k+i+1}{ }^{\perp}$ for $i<n-k$. Analogously, $M \cap U^{\perp} \leq M_{n-k}$ since $M \cap U^{\perp} \leq M_{0}$ and $M \cap U^{\perp} \leq p^{\perp}$ for every $p \in U$. Since $\left\langle p_{i} \mid k<i \leq n\right\rangle \subseteq$ $M_{n-k} \backslash M$ we obtain $\mathrm{d}^{*}\left(M, M_{n-k}\right)=n-k$. This $\operatorname{implies} \operatorname{crk}_{M}\left(M \cap U^{\perp}\right) \geq n-k$ since $M \cap U^{\perp} \leq M_{n-k}$. On the other hand $M \cap p^{\perp}$ is a hyperplane of $M$ for every $p \in U \backslash M$ and $U^{\perp}=\bigcap_{k<i \leq n} p_{i}{ }^{\perp}$. Therefore $\operatorname{crk}_{M}\left(M \cap U^{\perp}\right)=n-k$. This implies (i) and $M \cap U^{\perp}=M \cap M_{n-k}$. Since $\operatorname{crk}_{M_{n-k}}\left(M \cap M_{n-k}\right)=n-k$, we obtain $M_{n-k}=\left\langle U, M \cap M_{n-k}\right\rangle$ and the claim follows.

Proposition 2.13. In a non-degenerate polar space of infinite rank there are two generators $M$ and $N$ with $\mathrm{d}^{*}(M, N)=\infty$.

Proof. Let $M$ be a generator and let $\mathscr{G}$ be the set of all singular subspaces that are disjoint to $M$. We have to show that $\mathscr{G}$ contains an element with infinite rank. By Zorn's Lemma it suffices to show that $H \in \mathscr{G}$ with $\operatorname{rk}(H)<\infty$ is not a maximal element of $\mathscr{G}$. Set $M_{H}:=M \cap H^{\perp}$. Then $\operatorname{crk}_{M}\left(M_{H}\right)=\operatorname{rk}(H)+1$ by Lemma 2.12(i). Let $p$ be a point that is not collinear to all points of $M_{H}$. If $H \leq p^{\perp}$, then $\operatorname{rk}(\langle p, H\rangle)=\operatorname{rk}(H)+1$ and $M \cap\langle p, H\rangle^{\perp}<M_{H}$. Thus, $\langle p, H\rangle \cap M=\emptyset$ by Lemma 2.12(i) and we are done. Hence, we may assume $H \not \leq p^{\perp}$.

Set $G:=p \oplus H$ and $M_{G}:=M \cap G^{\perp}$. Since $p^{\perp} \cap H$ is hyperplane of $H$, we know that $G \cap H$ is common hyperplane of $G$ and $H$. Hence, Lemma 2.12(i) implies that $M_{H}$ is a hyperplane of $M \cap(G \cap H)^{\perp}$. Since $M_{H} \not \leq p^{\perp}$ and $G=\langle p, G \cap H\rangle$, we conclude that $M_{G}$ is a hyperplane of $M \cap(G \cap H)^{\perp}$. Thus $\operatorname{crk}_{M}\left(M_{H}\right)=\operatorname{crk}_{M}\left(M_{G}\right)$ and Lemma 2.12(i) implies $G \in \mathscr{G} \operatorname{since} \operatorname{rk}(G)=\operatorname{rk}(H)$.

Since $M_{H} \neq M_{G}$, there is a point $q \in M_{G} \backslash M_{H}$. Since $p^{\perp}$ and $q^{\perp}$ contain the hyperplane $G \cap H$ of $H$, there is a point $r \in p q$ with $H \leq r^{\perp}$. Since $r \sim q$, we obtain $r \notin H$ and hence, $\langle r, H\rangle$ is a singular subspace containing $H$ properly. This implies $r \neq q$ and hence $r^{\perp} \cap M=p^{\perp} \cap M$. Thus, $M_{H} \not \leq r^{\perp}$ and consequently, $\langle r, H\rangle^{\perp} \cap M$ is a hyperplane of $M_{H}$. By Lemma 2.12(i) this implies $\langle r, H\rangle \cap M=\emptyset$.

The consequence of this proposition is that the dual polar graph of a polar space with infinite rank is disconnected. In the finite rank case it is a consequence of Proposition 2.10 
that two generators have maximal distance in the dual polar graph if and only if they have empty intersection. In the infinite rank case the distance of two generators is infinite whenever the intersection of these two generators has finite rank. Hence, considering both the rank and the corank of the intersection gives more information than the distance in the dual polar graph. More precisely, we get a more detailed concept of distance in the dual polar graph, where distance $\infty$ between two points does not necessarily mean that these points are at maximal distance. Hence, in a dual polar space of a polar space with infinite rank there is information that cannot be expressed in terms of points, lines and incidence. Using an idea of B. Mühlherr, we equip point-line spaces with an extra point relation, called the opposition relation, which defines pairs of points to be at maximal distance, to characterise dual polar spaces.

\section{Local dual polar spaces}

Recall that for a set $M$ a relation $R \subset M \times M$ is called left-total, if $M=\{x \mid \exists y \in$ $M:(x, y) \in R\}$. Right-total is defined in the analogous way. A relation that is left-total and right-total is called total.

Definition 3.1. Let $(\mathcal{P}, \mathcal{L})$ be a partially linear space equipped with a symmetric, total point-relation $R \subset \mathcal{P} \times \mathcal{P}$. Then we call $\operatorname{cod}_{R}(x, y):=\min \{\mathrm{d}(z, y) \mid(x, z) \in R\}$ the $R$-codistance from $x$ to $y$.

Note that this definition is not symmetric. Later on we will use a point-relation $R$ for which the $R$-codistance is symmetric (see Proposition 3.8). Moreover, the used pointrelation should denote pairs of points which are in some sense at maximal distance. So the $R$-codistance is the minimal distance to a point at maximal distance. Thus, we visualise pairs of points with a greater $R$-codistance to be somehow closer. This motivates, unlike the distance, to define the codistance for two sets of points $X$ and $Y$ by $\operatorname{cod}_{R}(X, Y):=$ $\sup \left\{\operatorname{cod}_{R}(x, y) \mid x \in X \wedge y \in Y\right\}$.

Definition 3.2. Let $\mathscr{S}=(\mathcal{P}, \mathcal{L})$ be a partially linear space, $U \leq \mathscr{S}$ and $p \in \mathcal{P}$. If there is a unique point $q \in U$ with $\mathrm{d}(p, q)=\mathrm{d}(p, U)$, then we call $q$ the projection of $p$ in $U$ and write $\operatorname{pr}_{U}(p):=q$.

Let $R$ be a symmetric, total point-relation. If there is a unique point $r \in U$ with $\operatorname{cod}_{R}(p, r)=\operatorname{cod}_{R}(p, U)$, then we call $r$ the $R$-coprojection of $p$ in $U$ and write $\operatorname{copr}_{R, U}(p):=r$.

Definition 3.3. Let $\mathscr{S}$ be a partially linear space with a symmetric, total point relation $R$. Let $U$ and $V$ be two subspaces of $\mathscr{S}$ containing at least one line. We call $U$ parallel to $V$, if for every point $x \in U$ there is a unique point $\operatorname{pr}_{V}(x)$ with $\mathrm{d}\left(x, \operatorname{pr}_{V}(x)\right)=\mathrm{d}(U, V)$. We call $U R$-coparallel to $V$, if for every point $x \in U$ there is a unique point $\operatorname{copr}_{R, V}(x)$ with $\operatorname{cod}_{R}\left(x, \operatorname{copr}_{R, V}(x)\right)=\operatorname{cod}_{R}(U, V)$.

Note that our definitions of parallel and $R$-coparallel are not symmetric. The following definition plays a fundamental role in our paper. 
Definition 3.4. Let $\mathscr{S}=(\mathcal{P}, \mathcal{L})$ be a non-empty partially linear space. Further let $R$ be a symmetric, total point-relation with the property that for every set $\{x, y, z\} \subseteq \mathcal{P}$ with $\mathrm{d}(y, z)=n<\infty$ and $\min \left\{\operatorname{cod}_{R}(x, v) \mid v \in\langle y, z\rangle_{\mathrm{g}}\right\}=m<\infty$, there is a point $w \in \mathcal{P}$ with $\mathrm{d}(w, x)=n$ such that $\langle w, x\rangle_{\mathrm{g}}$ is $R$-coparallel to $\langle y, z\rangle_{\mathrm{g}}$ with $\operatorname{cod}_{R}\left(\langle w, x\rangle_{\mathrm{g}},\langle y, z\rangle_{\mathrm{g}}\right)=m+n$. Then we call $\mathscr{S}$ a local dual polar space (abbreviation: LDP) and the relation $R$ an opposition relation of $\mathscr{S}$.

Note that for an LDP there might be more than one opposition relation. Since mostly we pick just one opposition relation, denoted by $\leftrightarrow$, we drop the character $\leftrightarrow$ for the terms codistance, coprojection and coparallel. Thus, we write cod and $\operatorname{copr}_{X}$ instead of $\operatorname{cod}_{\leftrightarrow}$ and copr $\leftrightarrow_{\leftrightarrow, X}$. We call two points $x$ and $y$ of $\mathscr{S}$ opposite, if $x \leftrightarrow y$ holds. For two points $x$ and $y$ which are not opposite, we write $x \leftrightarrow y$.

Lemma 3.5. Let $\mathscr{S}$ be an LDP. Further let $x, y$ and $z$ be points of $\mathscr{S}$ with $\mathrm{d}(y, z)=$ $n<\infty$ and $\min \left\{\operatorname{cod}(x, v) \mid v \in\langle y, z\rangle_{\mathrm{g}}\right\}=m<\infty$. Then there exists a unique point $x^{\prime} \in\langle y, z\rangle_{\mathrm{g}}$ such that $\operatorname{cod}\left(x, x^{\prime}\right)=m+n=\operatorname{cod}\left(x,\langle y, z\rangle_{\mathrm{g}}\right)$.

Proof. This is a immediate consequence of Definition 3.4.

Lemma 3.6. Let $(\mathcal{P}, \mathcal{L})$ be an $L D P$. Then for any pair $(x, g) \in \mathcal{P} \times \mathcal{L}$ the point $x$ is non-opposite to either all points on $g$ or exactly one point on $g$.

Proof. Assume that there is a point $y \in g$ with $x \leftrightarrow y$. Choosing a second point $y^{\prime} \in$ $g \backslash\{y\}$ we obtain $g=\left\langle y, y^{\prime}\right\rangle_{\mathrm{g}}$. Hence by Definition 3.4, there is a point $x^{\prime} \sim x$ with $x \neq x^{\prime}$ such that the line $x x^{\prime}$ is coparallel to $g$ with $\operatorname{cod}\left(x x^{\prime}, g\right)=1$. Hence there is exactly one point, namely $\operatorname{copr}_{g}(x)$, on $g$ at codistance $\operatorname{cod}(x, g)=1$ to $x$.

Lemma 3.7. Let $x$ and $y$ be points of an LDP with $x \leftrightarrow y$. Then for every line $h$ through $y$ there is a line $g$ through $x$ and a bijective map $\varphi: g \rightarrow h$ such that $u \leftrightarrow v \Leftrightarrow u^{\varphi}=v$ for all $(u, v) \in g \times h$.

Proof. Let $h$ be a line through $y$. Then $h=\left\langle y, y^{\prime}\right\rangle_{\mathrm{g}}$ for a point $y^{\prime} \in h \backslash\{y\}$. By Definition 3.4 there is a point $x^{\prime} \sim x$ such that $g:=x x^{\prime}$ is coparallel to $h$ with $\operatorname{cod}(g, h)=1$. Hence, $\operatorname{copr}_{h}(u)$ is the only non-opposite point on $h$ for a point $u \in g$. Thus, $\varphi: g \rightarrow$ $h: u \mapsto \operatorname{copr}_{h}(u)$ is the only map with $u \leftrightarrow u^{\varphi}$ for every $u \in g$. By Lemma 3.6 there is no point on $h$ that is opposite to all points of $g$. Therefore $\varphi$ has to be surjective. For the injectivity we assume that there are two different points $u$ and $v$ on $g$ with $u^{\varphi}=v^{\varphi}$. Then by Lemma 3.6 there is no point on $g$ opposite $u^{\varphi}$ and hence $g^{\varphi}=\left\{u^{\varphi}\right\}$. This is a contradiction to $g^{\varphi}=h$.

Proposition 3.8. Let $x$ and $y$ be points of an LDP. Then $\operatorname{cod}(x, y)=\operatorname{cod}(y, x)$.

Proof. Assume $\operatorname{cod}(x, y)=m<\infty$. Let $\left(y_{i}\right)_{0 \leq i \leq m}$ be a path such that $y_{m}=y$ and $y_{0} \leftrightarrow x$. It suffices to prove by induction that for $i \leq m$ there is a point $x_{i}$ with $x_{i} \leftrightarrow y_{i}$ and $\mathrm{d}\left(x, x_{i}\right) \leq i$. Set $x_{0}:=x$. If the claim holds for $i<m$, then there is a point $x_{i+1} \sim x_{i}$ with $x_{i+1} \leftrightarrow y_{i+1}$ by Lemma 3.7. Thus, $\mathrm{d}\left(x, x_{i+1}\right) \leq i+1$ and therefore $x_{m} \leftrightarrow y$ with $\mathrm{d}\left(x, x_{m}\right) \leq m$. 
This proposition helps us to state a list of rather strong properties of LDPs that are much catchier than Definition 3.4.

Proposition 3.9. Let $w, x, y$ and $z$ be points of an LDP such that $\mathrm{d}(w, x)=\mathrm{d}(y, z)=$ $n<\infty$ and $U:=\langle w, x\rangle_{\mathrm{g}}$ is coparallel to $V:=\langle y, z\rangle_{\mathrm{g}}$ with $\operatorname{cod}(U, V)=m+n<\infty$. Then:

(i) $\min \{\operatorname{cod}(u, v) \mid u \in U, v \in V\}=m$.

(ii) $\forall u \in U \exists v \in V: \operatorname{cod}(u, v)=m$ and $\forall v \in V \exists u \in U: \operatorname{cod}(u, v)=m$.

(iii) $V$ is coparallel to $U$.

(iv) There is a unique bijective map $\varphi: U \rightarrow V$ with $\operatorname{cod}\left(u, u^{\varphi}\right)=m+n$ for every $u \in U$. Furthermore $u^{\varphi}=\operatorname{copr}_{V}(u)$ for $u \in U$ and $v=\operatorname{copr}_{U}(v)^{\varphi}$ for $v \in V$.

Proof. Let $u \in U$. We substitute in Definition 3.4 the points $x, y$ and $z$ by $u, y$ and $z$. Then $\operatorname{cod}\left(u, \operatorname{copr}_{V}(u)\right)=m+n$ implies $\min \{\operatorname{cod}(u, v) \mid v \in V\}=m$. This proves (i) and the first part of (ii). Now let $v \in V$ and substitute the points $x, y$ and $z$ in Definition 3.4 by $v, x$ and $w$. With $\operatorname{cod}(V, U)=n+m$ and (i) this implies $\min \{\operatorname{cod}(v, u) \mid u \in U\}=m, \operatorname{cod}(v, U)=n+m$ and that $\operatorname{copr}_{U}(v)$ exists. This proves (ii) and (iii). Now $\operatorname{cod}\left(u, u^{\varphi}\right)=m+n$ implies $u^{\varphi}=\operatorname{copr}_{V}(u)$ and hence $\varphi$ is unique. Finally, $v=\operatorname{copr}_{U}(v)^{\varphi}$ for all $v \in V$ and the bijectivity of $\varphi$ follows with $\operatorname{copr}_{U}\left(\operatorname{copr}_{V}(u)\right)=u$ and $\operatorname{copr}_{V}\left(\operatorname{copr}_{U}(v)\right)=v$.

In an LDP the convex subspaces play a major role. We first study convex spans of two points; it will turn out that all convex subspaces with finite diameter are of this type, see Corollary 3.17 .

Lemma 3.10. Let $y$ and $z$ be two points of an LDP. Then $\operatorname{diam}\left(\langle y, z\rangle_{\mathrm{g}}\right)=\mathrm{d}(y, z)$.

Proof. For $\mathrm{d}(y, z)=\infty$ there is nothing to prove. Thus, set $n:=\mathrm{d}(y, z)$ and $V:=$ $\langle y, z\rangle_{\mathrm{g}}$. Let $u$ and $v$ be two points of $V$ at maximal distance $k \geq n$. Further let $x$ be a point with $x \leftrightarrow u$. Then $\operatorname{cod}(x, V)=n$ and $\operatorname{cod}\left(x,\langle u, v\rangle_{\mathrm{g}}\right)=k$ by Lemma 3.5. With $\langle u, v\rangle_{\mathrm{g}} \leq V$ we conclude $k \leq n$.

By Proposition 3.9 there is for every subspace $V:=\langle y, z\rangle_{\mathrm{g}}$ a subspace $U=\langle w, x\rangle_{\mathrm{g}}$ such that $V$ and $U$ are coparallel to each other with $\operatorname{cod}(U, V)=\operatorname{diam}(V)=\operatorname{diam}(U)$. We will call two subspaces with this condition opposite.

Proposition 3.11. Let $y$ and $z$ be two points of an $L D P$ with $\mathrm{d}(y, z)=n$. Then for every $u \in\langle y, z\rangle_{\mathrm{g}}$ there is a point $v \in\langle y, z\rangle_{\mathrm{g}}$ with $\mathrm{d}(u, v)=n$. Moreover, $\langle u, v\rangle_{\mathrm{g}}=\langle y, z\rangle_{\mathrm{g}}$.

Proof. Let $X$ be some subspace opposite $V:=\langle y, z\rangle_{\mathrm{g}}$ and let $u \in V$. Take a point $u^{\prime} \in$ $X$ with $u^{\prime} \leftrightarrow u$ and set $v:=\operatorname{copr}_{U}\left(u^{\prime}\right)$. Then $\mathrm{d}(u, v)=n$ because of $\operatorname{cod}\left(u^{\prime}, v\right)=n$ and Lemma 3.10. Now $\operatorname{cod}\left(x,\langle u, v\rangle_{\mathrm{g}}\right) \geq n$ for every $x \in X$ by Lemma 3.5. Hence, $\operatorname{cod}\left(x, \operatorname{copr}_{V}(x)\right)=n$ implies $\operatorname{copr}_{V}(x) \in\langle u, v\rangle_{\mathrm{g}}$. The claim follows since $\varphi: X \rightarrow$ $V: x \mapsto \operatorname{copr}_{V}(x)$ is bijective. 
Definition 3.12. Let $U$ be a connected subspace of an LDP. Further let $p$ and $p^{\prime}$ be points with $\mathrm{d}(p, U)<\infty$ and $\operatorname{cod}\left(p^{\prime}, U\right)<\infty$.

If there is a point $q \in U$ such that for every point $r \in U$ there is a geodesic from $r$ to $p$ passing $q$, we call $q$ the gate of $p$ in $U$. If there is a point $q^{\prime} \in U$ with $\operatorname{cod}\left(p^{\prime}, r\right)=$ $\operatorname{cod}\left(p^{\prime}, q^{\prime}\right)-\mathrm{d}\left(q^{\prime}, r\right)$ for every point $r \in U$, we call $q^{\prime}$ the cogate of $p^{\prime}$ in $U$.

If for every point $r$ with $\mathrm{d}(r, U)<\infty$ the gate of $r$ in $U$ exists, we call $U$ gated. Analogously, if for every point $r$ with $\operatorname{cod}(r, U)<\infty$ the cogate of $r$ in $U$ exists, we call $U$ cogated.

We do not introduce any notation for the gate and the cogate of a point since if the (co)gate exists it coincides with the (co)projection.

Proposition 3.13. In an LDP the convex span of two points at finite distance is cogated.

Proof. Let $y$ and $z$ be two points at finite distance and set $V:=\langle y, z\rangle_{\mathrm{g}}$. Further let $x$ be a point with $\operatorname{cod}(x, V)<\infty$ and set $x^{\prime}:=\operatorname{copr}_{V}(x)$. We prove the claim by showing that for every point $u \in V \backslash\left\{x^{\prime}\right\}$ with $\operatorname{cod}(x, u)=k$ there is a point $u^{\prime} \in V$ collinear to $u$ with $\operatorname{cod}\left(x, u^{\prime}\right)=k+1$. Since $V$ has finite diameter and $\mathrm{d}\left(x^{\prime}, v\right) \geq \operatorname{cod}\left(x, x^{\prime}\right)-\operatorname{cod}(x, v)$ for every point $v \in V$, this implies by induction that there is a finite sequence $\left(u_{i}\right)_{0 \leq i \leq m}$ of points of $V$ such that $\mathrm{d}\left(u, u_{i}\right)=i, \operatorname{cod}\left(x, u_{i}\right)=k+i$ and $u_{m}=x^{\prime}$.

Let $u \in V \backslash\left\{x^{\prime}\right\}$. Further let $v \in V$ be a point at minimal distance to $u$ satisfying $\operatorname{cod}(x, u)<\mathrm{d}(v, u)+\operatorname{cod}(x, v)$. Since $x^{\prime} \in V$, such a point exists. Let $\left(u_{i}\right)_{0 \leq i \leq s}$ be a geodesic from $u_{0}=u$ to $u_{s}=v$. Since $V$ is convex, $u_{i} \in V$. Assume $\operatorname{cod}(x, v)=\operatorname{cod}\left(x, u_{s-1}\right)$. Then by Lemma 3.5 there is some point $v^{\prime}$ on the line $u_{s-1} v$ with $\operatorname{cod}\left(x, v^{\prime}\right)=\operatorname{cod}(x, v)+1$. Since $\mathrm{d}\left(v^{\prime}, u\right) \geq \mathrm{d}(v, u)-1$ and $\operatorname{cod}\left(x, v^{\prime}\right)=$ $\operatorname{cod}(x, v)+1$, we obtain $\operatorname{cod}(x, u)<\mathrm{d}\left(v^{\prime}, u\right)+\operatorname{cod}\left(x, v^{\prime}\right)$. By the convexity of $V$ we conclude $v^{\prime} \in V$ and therefore $\mathrm{d}\left(v^{\prime}, u\right)=s$. Hence, we may assume $\operatorname{cod}(x, v)=$ $\operatorname{cod}\left(x, u_{s-1}\right)+1$.

We may assume $s>1$ since otherwise we are done. Then $\operatorname{cod}\left(x, u_{s-2}\right)=\operatorname{cod}\left(x, u_{s}\right)$ holds. Using Lemma 3.5 we find a unique point $u^{\prime} \in\left\langle u_{s-2}, v\right\rangle_{\mathrm{g}} \leq V$ with greatest codistance to $x$. Since $u_{s-2} \neq u_{s}$, we obtain $\operatorname{cod}(x, v)<\operatorname{cod}\left(x, u^{\prime}\right)$. Lemma 3.10 implies $\mathrm{d}\left(u^{\prime}, v\right) \leq 2$ and hence $\mathrm{d}\left(u^{\prime}, u\right) \geq s-2$. Therefore, $\mathrm{d}\left(u^{\prime}, u\right)+\operatorname{cod}\left(x, u^{\prime}\right)>$ $\mathrm{d}\left(u_{s-2}, u\right)+\operatorname{cod}\left(x, u_{s-2}\right)=\operatorname{cod}(x, u)$. Hence, $\mathrm{d}\left(u^{\prime}, u\right) \geq s$ by the minimality of $s$. Since $\mathrm{d}\left(u_{s-2}, u^{\prime}\right) \leq 2$ by Lemma 3.10, we obtain $\mathrm{d}\left(u^{\prime}, u\right)=s$ and $\mathrm{d}\left(u^{\prime}, u_{s-2}\right)=2$. Let $v^{\prime}$ be a point collinear to $u^{\prime}$ and $u_{s-2}$. Then $\mathrm{d}\left(v^{\prime}, u\right)=s-1$ and $\operatorname{cod}\left(x, v^{\prime}\right) \geq \operatorname{cod}\left(x, u^{\prime}\right)-$ $1 \geq \operatorname{cod}\left(x, u_{s-2}\right)$. Thus, $\mathrm{d}\left(v^{\prime}, u\right)+\operatorname{cod}\left(x, v^{\prime}\right)>\mathrm{d}\left(u_{s-2}, u\right)+\operatorname{cod}\left(x, u_{s-2}\right)=\operatorname{cod}(x, u)$, a contradiction to the minimality of $s$.

Corollary 3.14. For $i \in\{0,1\}$ let $y_{i}$ and $z_{i}$ be points of an LDP with $\mathrm{d}\left(y_{i}, z_{i}\right)=n$ such that $U:=\left\langle y_{0}, z_{0}\right\rangle_{\mathrm{g}}$ and $V:=\left\langle y_{1}, z_{1}\right\rangle_{\mathrm{g}}$ are coparallel to each other. Then $\varphi: U \rightarrow$ $V: u \mapsto \operatorname{copr}_{V}(u)$ is an isomorphism.

Proof. By Proposition $3.9 \varphi$ is bijective. Set $m:=\operatorname{cod}(U, V)$ and let $u$ and $v$ be two 
distinct collinear points in $U$. Then Proposition 3.13 implies

$$
\begin{aligned}
\mathrm{d}\left(\operatorname{copr}_{V}(u), \operatorname{copr}_{V}(v)\right) & =\operatorname{cod}\left(u, \operatorname{copr}_{V}(u)\right)-\operatorname{cod}\left(u, \operatorname{copr}_{V}(v)\right) \\
& =\operatorname{cod}\left(u, \operatorname{copr}_{V}(u)\right)-\left(\operatorname{cod}\left(v, \operatorname{copr}_{V}(v)\right)-\mathrm{d}(v, u)\right)=1
\end{aligned}
$$

Thus, $u^{\varphi} \sim v^{\varphi}$. Now let $w \in u v \backslash\{u, v\}$. Then $\operatorname{cod}\left(w, \operatorname{copr}_{V}(u)\right)=\operatorname{cod}\left(w, \operatorname{copr}_{V}(v)\right)$ $=m-1$ by Proposition 3.13. By Lemma 3.5 we find a unique point on $u^{\varphi} v^{\varphi}$ with maximal codistance to $w$ and hence $\operatorname{copr}_{V}(w) \in u^{\varphi} v^{\varphi}$. Thus, $(u v)^{\varphi} \subseteq u^{\varphi} v^{\varphi}$. Exchanging $U$ and $V$ finishes the proof.

Lemma 3.15. Let $M \subseteq \mathcal{P}$ be a finite set of points of an LDP such that $\mathrm{d}(u, v)<\infty$ for every two points $u$ and $v$ of $M$. Then there are points $y$ and $z$ at finite distance such that $M \subseteq\langle y, z\rangle_{\mathrm{g}}$.

Proof. We may assume $|M| \geq 3$ since otherwise there is nothing to prove. Take two points $u$ and $v$ of the set $M$ and set $n:=\mathrm{d}(u, v)$. Set $V:=\langle u, v\rangle_{\mathrm{g}}$. We may assume that there is a point $w \in M \backslash V$ since otherwise we are done. Since $|M|$ and $\mathrm{d}(w, V)$ are finite it suffices to show that we find points $y$ and $z$ at finite distance such that $V \leq\langle y, z\rangle_{\mathrm{g}}$ and $\mathrm{d}\left(w,\langle y, z\rangle_{\mathrm{g}}\right)<\mathrm{d}(w, V)$.

Let $w_{0} \in V$ be a point with $\mathrm{d}\left(w, w_{0}\right)=\mathrm{d}(w, V)$. Further let $w_{1}$ be a point with $w_{0} \sim w_{1}$ and $\mathrm{d}\left(w_{1}, w\right)=\mathrm{d}\left(w_{0}, w\right)-1$ and let $y \in V$ be a point at distance $n$ to $w_{0}$. Now let $X$ be some subspace opposite $V$. Set $x:=\operatorname{copr}_{X}(y)$. Then $x \leftrightarrow w_{0}$ by Proposition 3.13. Let $z$ be the unique point on $w_{0} w_{1}$ with $\operatorname{cod}(z, x)=1$. Then $z \neq w_{0}$ and hence $z \notin V$ since $w_{1} \notin V$. This implies $\mathrm{d}(y, z) \geq n$ since otherwise there would be a geodesic from $y$ to $w_{0}$ passing $z$. Assume $\mathrm{d}(y, z)=n+1$. Then $\langle y, z\rangle_{\mathrm{g}}$ contains $w_{0}$ and therefore also $V=\left\langle w_{0}, y\right\rangle_{\mathrm{g}}$ and $w_{1}$ since $w_{1} \in w_{0} z$.

Now assume $\mathrm{d}(y, z)=n$. Set $U:=\langle y, z\rangle_{\mathrm{g}}$ and $y^{\prime}:=\operatorname{copr}_{U}(x)$. Then $\mathrm{d}(z, y)+$ $\operatorname{cod}(z, x)=n+1 \neq \operatorname{cod}(y, x)$ implies $y \neq y^{\prime}$ by Proposition 3.13. Therefore we have $\operatorname{cod}\left(y^{\prime}, x\right)>\operatorname{cod}(y, x)$ and hence $\operatorname{cod}\left(y^{\prime}, x\right)=n+1 \operatorname{since} \operatorname{cod}(z, x)=1$ and $\mathrm{d}\left(z, y^{\prime}\right) \leq$ $n$. Thus, $\mathrm{d}\left(y^{\prime}, z\right)=n$ and consequently $\mathrm{d}\left(y^{\prime}, w_{0}\right)=n+1$, since $x \leftrightarrow w_{0}$. Hence, $z \in\left\langle y^{\prime}, w_{0}\right\rangle_{\mathrm{g}}$. Now Proposition 3.13 implies $\mathrm{d}\left(y^{\prime}, y\right)=1$ and therefore $y \in\left\langle y^{\prime}, w_{0}\right\rangle_{\mathrm{g}}$. Thus, $\left\langle y^{\prime}, w_{0}\right\rangle_{\mathrm{g}}$ contains $V$ and $w_{1}$.

Proposition 3.16. In an LDP the convex span of two points at finite distance is gated.

Proof. Let $y$ and $z$ be two points at finite distance and set $U:=\langle y, z\rangle_{\mathrm{g}}$. Now let $x$ be a point with $\mathrm{d}(x, U)<\infty$. By Lemma 3.15 we find two points $y^{\prime}$ and $z^{\prime}$ such that $\{x, y, z\} \subseteq\left\langle y^{\prime}, z^{\prime}\right\rangle_{\mathrm{g}}$. Let $V$ be a subspace opposite $\left\langle y^{\prime}, z^{\prime}\right\rangle_{\mathrm{g}}$. Further set $x^{\prime}:=\operatorname{copr}_{V}(x)$ and $u^{\prime}:=\operatorname{copr}_{U}\left(x^{\prime}\right)$. Then by Proposition 3.13 we obtain for any point $u \in U$ :

$$
\begin{aligned}
\mathrm{d}(x, u) & =\operatorname{cod}\left(x, x^{\prime}\right)-\operatorname{cod}\left(u, x^{\prime}\right) \\
& =\operatorname{cod}\left(x, x^{\prime}\right)-\left(\operatorname{cod}\left(u^{\prime}, x^{\prime}\right)-\mathrm{d}\left(u, u^{\prime}\right)\right) \\
& =\mathrm{d}\left(x, u^{\prime}\right)+\mathrm{d}\left(u, u^{\prime}\right)
\end{aligned}
$$

Hence, $u^{\prime}$ is the gate for $x$ in $U$. 
Corollary 3.17. In an LDP every non-empty convex subspace $U$ with finite diameter is the convex span of two points.

Proof. We take two points $x$ and $y$ in $U$ at maximal distance. Let $z$ be a point with $z \notin\langle x, y\rangle_{\mathrm{g}}$ and $\mathrm{d}(x, z)<\infty$. By Proposition 3.11 we may assume $y=\operatorname{pr}_{\langle x, y\rangle_{\mathrm{g}}}(z)$. Since $\mathrm{d}(y, z) \geq 1$ Proposition 3.16 leads to $\mathrm{d}(x, z)=\mathrm{d}(x, y)+\mathrm{d}(y, z) \geq n+1$. Hence, $z \notin U$ and therefore $U=\langle x, y\rangle_{\mathrm{g}}$.

The convex subspaces of finite diameter of an LDP $\mathscr{S}$ together with $\mathscr{S}$ form a lattice in the canonical way. We denote this lattice by $\mathscr{G}_{0}(\mathscr{S})$. By Lemma 3.10 we know that whenever there is a pair of points at distance $n$, there are convex subspaces of diameter $k$ for every $k \leq n$. The set of convex subspaces of $\mathscr{S}$ with diameter $k \in \mathbb{N}$ will be denoted by $\mathcal{U}_{k}(\mathscr{S})$. If there is no danger of confusion we will simply write $\mathscr{G}_{0}$ and $\mathcal{U}_{k}$.

\section{Local dual polar spaces with finite diameter}

We now focus on the distinct possible choices of the opposition relation for LDPs with finite diameter and show that there is always an opposition relation with some nice additional properties.

Lemma 4.1. Let $\mathscr{S}$ be an LDP with finite diameter. Then $\mathscr{S} \rightarrow \mathscr{S}: x \mapsto \operatorname{copr}_{\mathscr{S}}(x)$ is an automorphism.

Proof. We may assume $\mathscr{S} \neq \emptyset$ since otherwise there is nothing to prove. Let $y$ and $z$ be two points with $\mathrm{d}(y, z)=\operatorname{diam}(\mathscr{S})$. Then $\langle y, z\rangle_{\mathrm{g}}=\mathscr{S}$ by Corollary 3.17. Set $x:=z$. Then the condition in Definition 3.4 is satisfied since $\leftrightarrow$ is total. Hence, there is a point $w$ with $\mathrm{d}(w, x)=n$ such that $\langle w, x\rangle_{\mathrm{g}}$ is coparallel to $\mathscr{S}$. Since $\langle w, x\rangle_{\mathrm{g}}=\mathscr{S}$ the claim follows from Corollary 3.14 .

Proposition 4.2. Let $\mathscr{S}=(\mathcal{P}, \mathcal{L})$ be an $L D P$ with finite diameter. Then $R:=\{(x, y) \in$ $\mathcal{P} \times \mathcal{P} \mid \mathrm{d}(x, y)=\operatorname{diam}(\mathscr{S})\}$ is an opposition relation of $\mathscr{S}$. Moreover, for every opposition relation $Q$ of $\mathscr{S}$, there is an automorphism $\tau \in \operatorname{Aut}(\mathscr{S})$ such that $(x, y) \in$ $Q \Leftrightarrow\left(x, y^{\tau}\right) \in R$.

Proof. Let $Q$ be an arbitrary opposition relation of $\mathscr{S}$. Further let $\bar{Q}:=\{(x, y) \in \mathcal{P} \times \mathcal{P} \mid$ $\left.\left(x, \operatorname{copr}_{Q, \mathscr{S}}(y)\right) \in Q\right\}$. Note that $\tau: \mathscr{S} \rightarrow \mathscr{S}: x \mapsto \operatorname{copr}_{Q, \mathscr{S}}(x)$ is an involutory automorphism. Let $x$ and $y$ be points with $\operatorname{cod}_{Q}(x, y)=k$ and let $z$ be a point with $\mathrm{d}(y, z)=k$ and $(x, z) \in Q$. Then $\mathrm{d}\left(y^{\tau}, z^{\tau}\right)=k$ and since $\left(x, z^{\tau}\right) \in \bar{Q}$ we obtain $k^{\prime}:=\operatorname{cod}_{\bar{Q}}\left(x, y^{\tau}\right) \leq k$. Now let $z^{\prime}$ be a point at distance $k^{\prime}$ to $y^{\tau}$ with $\left(x, z^{\prime}\right) \in \bar{Q}$. Then $\left(x, z^{\prime \tau}\right) \in Q$ and as above $\mathrm{d}\left(y, z^{\prime \tau}\right)=k^{\prime}$. We conclude $\operatorname{cod}_{Q}(x, y)=\operatorname{cod}_{\bar{Q}}\left(x, y^{\tau}\right)$.

Now let $x, y$ and $z$ be three points of $\mathscr{S}$. Set $n:=\mathrm{d}(y, z)$ and $m:=\min \left\{\operatorname{cod}_{\bar{Q}}(x, v) \mid\right.$ $\left.v \in\langle y, z\rangle_{\mathrm{g}}\right\}$. Then $m=\min \left\{\operatorname{cod}_{Q}\left(x^{\tau}, v\right) \mid v \in\langle y, z\rangle_{\mathrm{g}}\right\}$ and hence by Definition 3.4 there is a point $w$ with $\mathrm{d}\left(w, x^{\tau}\right)=n$ such that $\left\langle w, x^{\tau}\right\rangle_{\mathrm{g}}$ is $Q$-coparallel to $\langle y, z\rangle_{\mathrm{g}}$ with $\operatorname{cod}_{Q}\left(\left\langle w, x^{\tau}\right\rangle_{\mathrm{g}},\langle y, z\rangle_{\mathrm{g}}\right)=m+n$. Hence, $\left\langle w^{\tau}, x\right\rangle_{\mathrm{g}}$ is $\bar{Q}$-coparallel to $\langle y, z\rangle_{\mathrm{g}}$ and $\operatorname{cod}_{\bar{Q}}\left(\left\langle w^{\tau}, x\right\rangle_{\mathrm{g}},\langle y, z\rangle_{\mathrm{g}}\right)=m+n$. Thus, $\bar{Q}$ is an opposition relation of $\mathscr{S}$. 
Proposition 3.13 implies $\operatorname{cod}_{\bar{Q}}(x, y)=\operatorname{cod}_{Q}\left(x, y^{\tau}\right)=\operatorname{cod}_{Q}\left(y, \operatorname{copr}_{Q, \mathscr{S}}(y)\right)-$ $\mathrm{d}(y, x)=\operatorname{diam}(\mathscr{S})-\mathrm{d}(x, y)$. Hence, $\bar{Q}=R$.

A consequence of this proposition is that for an LDP $\mathscr{S}$ with finite diameter, there is no need to use an opposition relation other than $R:=\{(x, y) \in \mathcal{P} \times \mathcal{P} \mid \mathrm{d}(x, y)=$ $\operatorname{diam}(\mathscr{S})\}$. We call $R$ the standard opposition relation. Since our motivation to introduce an opposition relation was denoting pairs of points at maximal distance, this result is just what we wanted.

Lemma 4.3. Let $x$ and $y$ be two points of an LDP with $\mathrm{d}(x, y)=n$. Further let $z$ be a point with $\mathrm{d}\left(\langle x, y\rangle_{\mathrm{g}}, z\right)=k$. Then $\operatorname{diam}\left(\langle x, y, z\rangle_{\mathrm{g}}\right)=k+n$.

Proof. Set $z^{\prime}:=\operatorname{pr}_{\langle x, y\rangle_{\mathrm{g}}}(z)$ and let $w \in\langle x, y\rangle_{\mathrm{g}}$ be a point with $\mathrm{d}\left(w, z^{\prime}\right)=n$. Then $z^{\prime} \in$ $\langle w, z\rangle_{\mathrm{g}}$ and $\mathrm{d}(w, z)=k+n$ by Proposition 3.16. Hence, $\langle x, y\rangle_{\mathrm{g}}=\left\langle w, z^{\prime}\right\rangle_{\mathrm{g}} \leq\langle w, z\rangle_{\mathrm{g}}$ and therefore $\langle x, y, z\rangle_{\mathrm{g}}=\langle w, z\rangle_{\mathrm{g}}$.

Lemma 4.4. Let $n \in \mathbb{N}$ and let $U \in \mathcal{U}_{n}$ be a subspace of an LDP. Further let $H$ and $V$ be two convex subspaces of $U$ where $\operatorname{diam}(H)=n-1$. Then $V \leq H$ or $V \cap H=\emptyset$ or $V \cap H \in \mathcal{U}_{\operatorname{diam}(V)-1}$.

Proof. Let $V \cap H \neq \emptyset$ and $V \not 亡 H$. By Proposition 3.11 we may assume $V=\langle u, v\rangle_{\mathrm{g}}$ where $u \in H$ and $v \notin H$. Since $V$ is convex and of finite diameter, we conclude $\operatorname{pr}_{H}(v) \in$ $V$ by Proposition 3.16. Hence, $\operatorname{diam}(V \cap H) \geq \mathrm{d}\left(u, \operatorname{pr}_{H}(v)\right)=\operatorname{diam}(V)-1$. Since $V \cap H$ is convex and $V \cap H \neq V$, this implies $\operatorname{diam}(V \cap H)=\operatorname{diam}(V)-1$ by Proposition 3.11.

Lemma 4.5. Let $n \in \mathbb{N}$ and let $U \in \mathcal{U}_{n}$ be a subspace of an LDP. Further let $H \leq U$ be a subspace with $H \in \mathcal{U}_{n-1}$. Then for every point $p \in U \backslash H$ there is a subspace $J \in \mathcal{U}_{n-1}$ with $p \in J \leq U$ and $H \cap J=\emptyset$.

Proof. Set $p^{\prime}:=\operatorname{pr}_{H}(p)$. We choose a point $q \in U$ with $\mathrm{d}\left(p^{\prime}, q\right)=n$ and set $q^{\prime}:=$ $\operatorname{pr}_{H}(q)$. Since $\operatorname{diam}(H)=n-1$, we obtain $q \notin H$ and therefore $p \sim p^{\prime}$ and $q \sim q^{\prime}$ by Lemma 4.3. Now Proposition 3.16 implies $\mathrm{d}\left(p^{\prime}, q^{\prime}\right)=n-1$ and consequently $\mathrm{d}\left(p, q^{\prime}\right)=$ $n$. Set $r:=\operatorname{pr}_{q q^{\prime}}(p)$. Since $\mathrm{d}\left(p, q^{\prime}\right)=n$ and $\operatorname{diam}(U)=n$, we obtain $\mathrm{d}(p, r)=n-1$. Thus, $r \neq q^{\prime}$ and we conclude $r \notin H$ and $q^{\prime}=\operatorname{pr}_{H}(r)$. Set $J:=\langle p, r\rangle_{\mathrm{g}}$. Assume there is a point $s \in H \cap J$. Then $p^{\prime} \in\langle p, s\rangle_{\mathrm{g}} \leq J$ and $q^{\prime} \in\langle r, s\rangle_{\mathrm{g}} \leq J$ by Proposition 3.16. Hence, $J=\left\langle p^{\prime}, q^{\prime}\right\rangle_{\mathrm{g}}=H$ by Proposition 3.11, a contradiction to $p \in J \backslash H$.

Proposition 4.6. In an LDP every non-empty convex subspace with finite diameter is an LDP.

Proof. Let $U$ be a convex subspace of an LDP with $\operatorname{diam}(U)<\infty$. Further let $x, y$ and $z$ be points of $U$ and set $V:=\langle x, y, z\rangle_{\mathrm{g}}, m:=\mathrm{d}(y, z)$ and $n:=\operatorname{diam}(V)$. Then $\mathrm{d}\left(x,\langle y, z\rangle_{\mathrm{g}}\right)=n-m$ by Lemma 4.3. Let $V^{\prime}$ be a subspace opposite $V$ and set $x^{\prime}:=$ $\operatorname{copr}_{V^{\prime}}(x)$. Then $\operatorname{cod}\left(x^{\prime}, V\right)=n$ and since $\operatorname{copr}_{V}\left(x^{\prime}\right)=x$ we obtain $\operatorname{cod}\left(x^{\prime},\langle y, z\rangle_{\mathrm{g}}\right)=$ 
$m$ by Proposition 3.13. Let $w^{\prime}$ be a point with $\mathrm{d}\left(w^{\prime}, x^{\prime}\right)=m$ such that $\left\langle w^{\prime}, x^{\prime}\right\rangle_{\mathrm{g}}$ is coparallel to $\langle y, z\rangle_{\mathrm{g}}$.

Set $W:=\left\langle w^{\prime}, x^{\prime}\right\rangle_{\mathrm{g}}$ and $\varphi: W \rightarrow V: u \mapsto \operatorname{copr}_{V}(u)$. Since there is an opposite point in $\langle y, z\rangle_{\mathrm{g}} \leq V$ for every $u \in W$, we obtain $\operatorname{cod}(u,\langle y, z)\rangle_{\mathrm{g}}=m, \operatorname{cod}(u, V)=n$ and hence $\operatorname{copr}_{W}\left(u^{\varphi}\right)=u$. This implies with Proposition $3.13 \operatorname{cod}\left(u, v^{\varphi}\right)=n-\mathrm{d}(u, v)$ for every $v \in W$ and consequently $\mathrm{d}(u, v)=\mathrm{d}\left(u^{\varphi}, v^{\varphi}\right)$. Hence, $\varphi$ is an injective morphism since by Proposition 3.16 there are no triangles in an LDP. Assume $u \sim v$. Then for every point $p \in u^{\varphi} v^{\varphi} \backslash\left\{u^{\varphi}, v^{\varphi}\right\}$, we obtain $\operatorname{cod}(p, u)=\operatorname{cod}(p, v)=n-1$. Hence, there is a point $q \in u v$ with $q^{\varphi}=p$. This implies $W^{\varphi}=\langle w, x\rangle_{\mathrm{g}}$ where $w:=w^{\prime \varphi}$. Since every $v \in\langle x, w\rangle_{\mathrm{g}}$ is the cogate in $V$ for some $u \in W$ and $\operatorname{cod}\left(u,\langle y, z\rangle_{\mathrm{g}}\right)=m$, we obtain $\mathrm{d}\left(v,\langle y, z\rangle_{\mathrm{g}}\right)=n-m$. Thus, $\langle w, x\rangle_{\mathrm{g}}$ is parallel to $\langle y, z\rangle_{\mathrm{g}}$ and hence $R$-coparallel, where $R$ is the standard opposition relation of $U$. The claim follows by Definition 3.4.

In an LDP $\mathscr{S}$ with finite diameter $n$ we will sometimes write $\mathcal{U}^{k}(\mathscr{S})$ instead of $\mathcal{U}_{n-k}(\mathscr{S})$. Again we write $\mathcal{U}^{k}$ if it is clear to which LDP we refer.

Definition 4.7. Let $\mathscr{S}$ be an LDP with finite diameter. For every subspace $G \in \mathcal{U}^{2}$ set $[G]:=\left\{P \in \mathcal{U}^{1} \mid G<P\right\}$. We call the point-line space $\mathscr{D}(\mathscr{S}):=\left(\mathcal{U}^{1},\{[G] \mid \emptyset \neq G \in\right.$ $\left.\left.\mathcal{U}^{2}\right\}\right)$ the dual of $\mathscr{S}$.

For $\{P, Q\} \subseteq \mathcal{U}^{1}$, we will write $P \perp Q$ if $P=Q$ or $\emptyset \neq P \cap Q \in \mathcal{U}^{2}$. The subspaces contained in $\mathcal{U}^{1}$ are the maximal proper convex subspaces. Hence, dualising an LDP with finite diameter and dualising a polar space are quite similar. We just use convex instead of singular subspaces and the diameter of these subspace instead of the rank. By Lemma 4.4 two subspaces of $\mathcal{U}^{1}$ intersect in a subspace of $\mathcal{U}^{2}$ whenever they are distinct but not disjoint. Hence, $P \perp Q \Leftrightarrow P \cap Q \neq \emptyset$.

Theorem 4.8. The dual of an LDP with finite diameter $\geq 1$ is a non-degenerate polar space.

Proof. Let $\mathscr{S}$ denote an LDP with finite diameter. If $\operatorname{diam}(\mathscr{S})=1$, then $\mathscr{S}$ consists of a single line $l$ and $\mathscr{D}(\mathscr{S})=(\{\{p\} \mid p \in l\}, \emptyset)$. Hence, $\mathscr{D}(\mathscr{S})$ satisfies (BS) and is non-degenerate since $|l| \geq 2$. Now let $\operatorname{diam}(\mathscr{S}) \geq 2$ and denote $P^{\perp}:=\left\{Q \in \mathcal{U}^{1} \mid\right.$ $\left.P \cap Q \in \mathcal{U}^{2}\right\} \cup\{P\}$ for $P \in \mathcal{U}^{1}$.

Let $G \in \mathcal{U}^{2}$. Then there is a point $p \in \mathscr{S} \backslash G$ with $\mathrm{d}(G, p)=1$ and by Lemma 4.3 we obtain $P:=\langle p, G\rangle_{\mathrm{g}} \in \mathcal{U}^{1}$. Now let $q \in \mathscr{S} \backslash P$. Then by Lemma 4.5 there is a subspace $Q \in \mathcal{U}^{1}$ with $q \in Q$ and $Q \cap P=\emptyset$. Let $r^{\prime} \in G$ and set $r:=\operatorname{pr}_{Q}\left(r^{\prime}\right)$. Then Lemma 4.3 implies $\mathrm{d}\left(r, r^{\prime}\right)=1$ since $r^{\prime} \notin Q$ and hence $R:=\langle G, r\rangle_{\mathrm{g}} \in \mathcal{U}^{1}$. With $r \notin P$ we obtain $R \neq P$ and therefore $|[G]| \geq 2$. Thus, $\mathscr{D}(\mathscr{S})$ is a point-line space.

We prove the claim by showing that for every pair $(P, G) \in \mathcal{U}^{1} \times \mathcal{U}^{2}$ either $P^{\perp} \cap[G]$ contains exactly one element or $[G] \subseteq P^{\perp}$. First let $G \cap P \neq \emptyset$. Then every $Q \in[G]$ intersects $P$ and hence $[G] \subseteq P^{\perp}$. Now let $G \cap P=\emptyset$ and let $v$ and $u$ be points of $G$ with $\mathrm{d}(u, v)=n-2$. Set $u^{\prime}:=\operatorname{pr}_{P}(u)$. Then $u \sim u^{\prime}$ by Lemma 4.3. Since $u^{\prime} \notin G$, we obtain $u=\operatorname{pr}_{G}\left(u^{\prime}\right)$ and therefore $\mathrm{d}\left(v, u^{\prime}\right)=n-1$. Hence, $Q:=\left\langle v, u^{\prime}\right\rangle_{\mathrm{g}} \in \mathcal{U}^{1}$. Furthermore, $Q \in P^{\perp}$ since $u^{\prime} \in Q \cap P$ and $Q \in[G]$ since $u \in\left\langle v, u^{\prime}\right\rangle_{\mathrm{g}}$. Conversely, let 
$R \in[G] \cap P^{\perp}$. Then there is a point $w \in P \cap R$. With $u \in G \leq R$ we obtain $u^{\prime} \in R$ since $u^{\prime} \in\langle u, w\rangle_{\mathrm{g}} \leq R$. Thus, $Q=\left\langle u^{\prime}, G\right\rangle_{\mathrm{g}} \leq R$ and therefore $[G] \cap P^{\perp}=\{Q\}$.

For every $P \in \overline{\mathcal{U}}^{1}$, we find a point $p \in \mathscr{S} \backslash P$. Therefore the polar space $\mathscr{D}(\mathscr{S})$ is non-degenerate by Lemma 4.5 .

In the case $\operatorname{diam}(\mathscr{S})=0$ the LDP $\mathscr{S}$ is a singleton, $\mathcal{U}^{1}=\{\emptyset\}$ and $\mathcal{U}^{2}=\emptyset$. Hence $\mathscr{D}(\mathscr{S})$ is also a singleton, which is a degenerate polar space. The following theorem motivates the name local dual polar space.

Theorem 4.9. Every non-empty $L D P$ with finite diameter is a dual polar space.

Proof. Let $\mathscr{S}$ denote an LDP with finite diameter $n$. We show that $\mathscr{S}$ is isomorphic to the dual polar space of $\mathscr{D}(\mathscr{S})$. If $\mathscr{S}$ is a singleton, then $\mathscr{D}(\mathscr{S})=\{\emptyset\}$ and the claim holds. If $\mathscr{S}$ consists of a single line, then $\mathscr{D}(\mathscr{S})$ has as many points as $\mathscr{S}$ and no lines. Since all generators of $\mathscr{D}(\mathscr{S})$ have a common hyperplane, namely $\emptyset$, the claim follows. Hence, from now on we may assume $\operatorname{diam}(\mathscr{S}) \geq 2$.

Let $M$ be a generator of $\mathscr{D}(\mathscr{S})$. We show that there is exactly one point $m \in \mathcal{P}$ such that $Q \in M \Leftrightarrow m \in Q$ for all $Q \in \mathcal{U}^{1}$. Let $P \in M$ and choose a point $x \in P$. Set $S_{x}:=\bigcap\{Q \in M \mid x \in Q\}$. Then $S_{x}$ is non-empty and convex with $0 \leq \operatorname{diam}\left(S_{x}\right) \leq n$. Now let $P^{\prime} \in M$ and set $x^{\prime}:=\operatorname{pr}_{P^{\prime}}(x)$. Further let $Q \in M$ with $x \in Q$. Then there is a point $y \in P^{\prime} \cap Q$ since $M$ is singular. Thus, $x^{\prime} \in\langle x, y\rangle_{\mathrm{g}} \leq Q$ and therefore $\left\{x^{\prime}\right\} \leq S_{x^{\prime}} \leq S_{x}$, where $S_{x^{\prime}}:=\bigcap\left\{Q \in M \mid x^{\prime} \in Q\right\}$. Hence, $x \notin P^{\prime}$ implies $S_{x^{\prime}}<S_{x}$ and $\operatorname{diam}\left(S_{x}\right)=0$ implies $x \in P^{\prime}$. Since $\operatorname{diam}\left(S_{x}\right)<\infty$, we conclude by induction $S:=\bigcap_{Q \in M} Q \neq \emptyset$. Suppose $\operatorname{diam}(S)>0$. Let $l \leq S$ be a line and let $x \in l$. Further let $y \in \mathscr{S}$ be a point with $\mathrm{d}(y, x)=n$ and set $x^{\prime}:=\operatorname{pr}_{l}(y)$. Then $Q:=\left\langle x^{\prime}, y\right\rangle_{\mathrm{g}} \in \mathcal{U}^{1}$ and $Q \cap P \neq \emptyset$ for every $P \in M$. Hence, there is a singular subspace in $\mathscr{D}(\mathscr{S})$ containing $M$ and $Q$. Since $M$ is a generator, this implies $Q \in M$. This a contradiction since $\mathrm{d}(x, y)=n$ and therefore $x \notin Q$. Thus, $S$ is a singleton.

Conversely, let $x$ be a point of $\mathscr{S}$ and set $M_{x}:=\left\{P \in \mathcal{U}^{1} \mid x \in P\right\}$. Then any two distinct elements $P$ and $Q$ of $M_{x}$ are adjacent and $[P \cap Q] \leq M_{x}$ since $x \in P \cap Q$. Thus, $M_{x}$ is a singular subspace. For every $R \in \mathcal{U}^{1}$ with $x \notin R$ there is a $R^{\prime} \in M_{x}$ with $R \cap R^{\prime}=\emptyset$ by Lemma 4.5. Hence, $M_{x}$ is a maximal singular subspace of $\mathscr{D}(\mathscr{S})$ and mapping $x$ to $M_{x}$ yields a bijection between the points of $\mathscr{S}$ and the generators of $\mathscr{D}(\mathscr{S})$.

It remains to check whether this map is an isomorphism. Since in an LDP all lines are gated, a set of 3 pairwise collinear points has to be contained in a line. Thus, the lines in an LDP coincide with the maximal cliques of the collinearity graph. Hence, it suffices to show that the collinearity graph of $\mathscr{S}$ and the dual polar graph of $\mathscr{D}(\mathscr{S})$ are isomorphic, i.e. two distinct generators $M_{x}$ and $M_{y}$ intersect in a common hyperplane if and only if $x \sim y$. Let $x$ and $y$ be two points of $\mathscr{S}$ and assume $x \not y$. Since $\{x\}=\bigcap_{Q \in M_{x}} Q$ there is an element $P \in M_{x}$ with $y \notin P$. Then $y \sim y^{\prime}:=\operatorname{pr}_{P}(y)$ by Lemma 4.3 and hence $x \neq y^{\prime}$. By Proposition 4.6P is an LDP and therefore $\bigcap\left\{L \in \mathcal{U}^{1}(P) \mid x \in L\right\}=\{x\}$. Hence, there is an element $G \in \mathcal{U}^{2}$ with $x \in G<P$ and $y^{\prime} \notin G$. This implies $\mathrm{d}(y, G)=$ $\mathrm{d}\left(y, y^{\prime}\right)+\mathrm{d}\left(y^{\prime}, G\right) \geq 2$. Thus, $\langle y, G\rangle=\mathscr{S}$ by Lemma 4.3 and therefore $[G] \cap M_{y}=\emptyset$. Hence, $M_{x} \cap M_{y}$ is no hyperplane of $M_{x}$ since $[G] \leq M_{x}$. Now assume $x \sim y$ and let 
$G \in \mathcal{U}^{2}$ with $[G] \leq M_{x}$. If $y \in G$, we obtain $[G] \leq M_{y}$. If $y \notin G$, we obtain $\langle y, G\rangle \in \mathcal{U}^{1}$ by Lemma 4.3. Hence, $[G] \cap M_{y} \neq \emptyset$ and therefore $M_{x}$ and $M_{y}$ have a hyperplane in common.

\section{Atomic local dual polar spaces}

We call a subspace $U$ of a point-line space $\mathscr{S}$ a connected component if there is a point $p \in U$ such that for every point $q \in \mathscr{S}$, we have $q \in U$ if and only if $\mathrm{d}(p, q)<\infty$. Let $U$ and $V$ be connected components of an LDP which are not necessarily distinct.

Remark 5.1. Let $U$ and $V$ be connected components of an LDP. Assume there are points $x \in U$ and $y \in V$ with $x \leftrightarrow y$. Then $\operatorname{cod}(u, v)<\infty$ for every two points $u \in U$ and $v \in V$ since $\operatorname{cod}(u, v) \leq \mathrm{d}(x, u)+\mathrm{d}(v, y)$. Hence, there are points $u^{\prime} \leftrightarrow u$ and $v^{\prime} \leftrightarrow v$ with $\mathrm{d}\left(u^{\prime}, v\right)<\infty$ and $\mathrm{d}\left(v^{\prime}, u\right)<\infty$. This implies $u^{\prime} \in V$ and $v^{\prime} \in U$. Thus, if for one point in $U$ there is an opposite point in $V$, then every point in $U$ is opposite to some point in $V$.

Motivated by this remark we introduce a graph $\Gamma_{C}(\mathscr{S})$ whose vertex set is the set of connected components of an LDP $\mathscr{S}$. Two connected components $U$ and $V$ are connected by an edge in $\Gamma_{C}(\mathscr{S})$ whenever there are points $p \in U$ and $q \in V$ with $p \leftrightarrow q$. Note that $\Gamma_{C}(\mathscr{S})$ depends on the used opposition relation. We allow $\Gamma_{C}(\mathscr{S})$ to have loops, i.e. edges where the two endpoints are the same. Since $\leftrightarrow$ is total, every vertex of this graph is contained in at least one edge. There might be vertices which are only connected to themselves by a loop. Such a vertex will be called isolated.

Now we divide an LDP $\mathscr{S}$ with a given opposition relation $R$ into two parts. The first part $\mathscr{S}_{F}$ is the subspace on the set of points $\mathcal{P}_{F}:=\{p \in \mathcal{P} \mid \forall q \in \mathcal{P}:(p, q) \in R \Rightarrow$ $\mathrm{d}(p, q)<\infty\}$. In other words, the graph $\Gamma_{C}\left(\mathscr{S}_{F}\right)$ is the subgraph of $\Gamma_{C}(\mathscr{S})$ consisting of all isolated vertices, where $\Gamma_{C}(\mathscr{S})$ and $\Gamma_{C}\left(\mathscr{S}_{F}\right)$ refer to $R$. The second part is the subspace on the points $\mathcal{P} \backslash \mathcal{P}_{F}$, which we denote by $\mathscr{S}_{\infty}$. Hence, the graph $\Gamma_{C}\left(\mathscr{S}_{\infty}\right)$ is the subgraph of $\Gamma_{C}(\mathscr{S})$ on the non-isolated vertices. The two parts $\mathscr{S}_{F}$ and $\mathscr{S}_{\infty}$ have no connection at all, since for every pair of points $(p, q) \in \mathscr{S}_{F} \times \mathscr{S}_{\infty}$ we obtain $p \nsim q$ and $(p, q) \notin R$. Therefore we also split the relation $R$ into two parts, namely $R_{F}$ and $R_{\infty}$, which are the restrictions of $R$ on the point sets of $R_{F}$ and $R_{\infty}$, respectively.

Proposition 5.2. Let $R$ be an opposition relation of an LDP. Set $\bar{R}:=\left\{(x, y) \in R_{\infty} \mid\right.$ $\mathrm{d}(x, y)=\infty\} \cup R_{F}$. Then $\bar{R}$ is again an opposition relation.

Proof. Note that we obtain $\bar{R}$ out of $R$ by removing all pairs $(x, y)$ of $R$ that belong to a loop at a non-isolated vertex of $\Gamma_{C}$. Hence, $\bar{R}$ is symmetric and total; see Remark 5.1. Furthermore, $\operatorname{cod}_{R}(p, q)=\operatorname{cod}_{\bar{R}}(p, q)$ or $\operatorname{cod}_{R}(p, q)<\operatorname{cod}_{\bar{R}}(p, q)=\infty$ for every two points $p$ and $q$.

Now let $x, y$ and $z$ be points that satisfy the conditions in Definition 3.4 for $\bar{R}$. Then they also satisfy this condition for $R$ and we find a point $w$ with $\mathrm{d}(w, x)=\mathrm{d}(y, z)$ such that $\langle w, x\rangle_{\mathrm{g}}$ is $R$-coparallel to $\langle y, z\rangle_{\mathrm{g}}$. Let $U$ and $V$ be the connected components with 
$x \in U$ and $y \in V$. Since $\operatorname{cod}_{\bar{R}}(x, y)<\infty$, we know that $U$ and $V$ are joined by an edge in $\Gamma_{C}$ and all pairs of $R \cap(U \times V)$ are contained in $\bar{R}$. This implies $\operatorname{cod}_{R}(u, v)=$ $\operatorname{cod}_{\bar{R}}(u, v)$ for all pairs $(u, v) \in U \times V$. Thus, $\langle w, x\rangle_{\mathrm{g}}$ is $\bar{R}$-coparallel to $\langle y, z\rangle_{\mathrm{g}}$ with $\operatorname{cod}_{\bar{R}}\left(\langle w, x\rangle_{\mathrm{g}},\langle y, z\rangle_{\mathrm{g}}\right)=\mathrm{d}(y, z)+\min \left\{\operatorname{cod}_{\bar{R}}(x, v) \mid v \in\langle y, z\rangle_{\mathrm{g}}\right\}$.

We will call the opposition relation $\bar{R}$ the reduced opposition relation of $R$ or simply a reduced opposition relation. From now on we may assume that the used opposition relation is a reduced one and that for every isolated connected component of finite diameter, the induced opposition relation is the standard opposition relation.

Let $\mathscr{S}$ be an LDP with a reduced opposition relation. We call a subspace that is induced by a connected component of $\Gamma_{C}(\mathscr{S})$ an irreducible component of $\mathscr{S}$. If $\mathscr{S}$ is an irreducible component itself, we will call $\mathscr{S}$ irreducible. Since every irreducible component is an LDP itself that does not affect the others, we may restrict our study to irreducible LDPs. In the following we will concentrate on two kinds of irreducible LDPs.

Definition 5.3. We call an irreducible LDP that either has finite diameter or consists of exactly two connected components with infinite diameter an atomic local dual polar space (abbreviation: ADP).

Note that the definitions of irreducible and atomic local dual polar spaces rely on the used opposition relation, whereas for local dual polar spaces we just need the existence of any opposition relation.

If an irreducible LDP has more than one connected component, it is a direct consequence of Definition 3.4 that all of them have the same diameter. Hence, there are three kinds of irreducible LDPs which are not atomic. The first kind consists of more than two connected components with infinite diameter. Every connected component of such an LDP is contained in an ADP. The second kind consists of at least two connected components with finite diameter $n$. Every connected component of such an LDP is an ADP by Proposition 4.6. Moreover, by Corollary 3.17 and Proposition 3.9 all the connected components are isomorphic to each other. The third kind consist of one connected component of infinite diameter. In this case we make an isomorphic copy of the irreducible LDP to obtain two connected components. Then we take the pairs of the old opposition relation and replace one of the two points by its copy. Hence, every pair gives rise to two new pairs. The pairs of points we obtain by doing so define an opposition relation such that the provided new structure is an ADP. Thus, all connected components that can occur in an LDP can also occur in an ADP.

Note that $x \leftrightarrow y$ implies $\mathrm{d}(x, y)=\operatorname{diam}(\mathscr{S})$ for any two points $x$ and $y$ of an ADP. This is just what we intended when we introduced the opposition relation.

ADPs which do not have finite diameter, give rise to the dual of polar spaces of infinite rank. To prove this we first introduce some terminology for infinite objects that is analogous to the ones we used in the finite case.

Definition 5.4. Let $x$ and $y$ be two points of an LDP at finite codistance and let $\alpha$ and $\beta$ be two non-empty initial segments of $\mathbb{N}$, i.e. a (not necessarily proper) subset of $\mathbb{N}$ that contains all numbers $\leq n$ if it contains $n$. Further let $\left(x_{i}\right)_{i \in \alpha}$ and $\left(y_{i}\right)_{i \in \beta}$ be two paths 
where $x_{0}=x$ and $y_{0}=y$ such that $\operatorname{cod}(x, y)+i+j=\operatorname{cod}\left(x_{i}, y_{j}\right)$ for all $(i, j) \in \alpha \times \beta$. Then we call $\left(\left(x_{i}\right)_{i \in \alpha},\left(y_{i}\right)_{i \in \beta}\right)$ a partial cogeodesic from $x$ to $y$.

The condition $\operatorname{cod}(x, y)+i+j=\operatorname{cod}\left(x_{i}, y_{j}\right)$ for all $(i, j) \in \alpha \times \beta$ implies that the paths $\left(x_{k}\right)_{0 \leq k \leq i}$ and $\left(y_{k}\right)_{0 \leq k \leq j}$ must be geodesics. A cogeodesic $\left(\left(x_{i}^{\prime}\right)_{i \in \alpha^{\prime}},\left(y_{i}^{\prime}\right)_{i \in \beta^{\prime}}\right)$ is contained in a cogeodesic $\left(\left(x_{i}\right)_{i \in \alpha},\left(y_{i}\right)_{i \in \beta}\right)$ if $\alpha^{\prime} \leq \alpha, \beta^{\prime} \leq \beta$ and $\left(x_{i}^{\prime}, y_{j}^{\prime}\right)=\left(x_{i}, y_{j}\right)$ for every $(i, j) \in \alpha^{\prime} \times \beta^{\prime}$. We call a partial cogeodesic from $x$ to $y$, which is not properly contained in any other partial cogeodesic from $x$ to $y$, a cogeodesic. Let $I$ be an index set and let $\left(s_{i}\right)_{i \in I}$ be a chain of cogeodesics between two points $x$ and $y$. Then the union ${ }^{2}$ of $\left(s_{i}\right)_{i \in I}$ is again a partial cogeodesic from $x$ to $y$. Hence, we may apply Zorn's Lemma to conclude that for every two points at finite codistance, there is a cogeodesic between them. In an LDP of finite diameter every cogeodesic is of the kind $\left(\left(x_{i}\right)_{0 \leq i \leq l},\left(x_{i}\right)_{n \geq i \geq l}\right)$, where $l \leq n$ and $\left(x_{i}\right)_{0 \leq i \leq n}$ is a geodesic.

Definition 5.5. Let $U$ be a convex subspace of an ADP such that for any two points $x$ and $y$ of $U$ with $\operatorname{cod}(x, y)<\infty$ all cogeodesics from $x$ to $y$ are contained in $U$. Then we call U coconvex.

Let $\mathfrak{G}$ be the set of all coconvex subspaces of an ADP $\mathscr{S}$. For a subset $X \subseteq \mathscr{S}$ we call $\langle X\rangle_{\mathrm{g}}:=\bigcap\{\mathcal{C} \mid X \subseteq \mathcal{C} \in \mathfrak{G}\}$ the coconvex span of $X$. Since $\mathscr{S}$ is coconvex, this intersection is well defined. For points $x_{0}, \ldots, x_{n}$ and a set of points $X$ we will write $\left\langle x_{0}, \ldots, x_{n}, X\right\rangle_{\mathrm{g}}$ rather than $\left\langle\left\{x_{0}, \ldots, x_{n}\right\} \cup X\right\rangle_{\mathrm{g}}$. If $X$ is contained in a connected component, then $\left\langle\langle X\rangle_{\mathrm{g}}\right.$ coincides with $\langle X\rangle_{\mathrm{g}}$.

Beside the convex subspaces of finite diameter there is another kind of convex subspaces that plays a major role in ADPs, namely the subspaces that are coconvex spans of two points at finite codistance. Before studying them we show how they look; see Lemma 5.8. To do so, we first need two quite technical lemmata.

Lemma 5.6. Let $y$ and $z$ be two points of an LDP with $\mathrm{d}(y, z)=n$. Further let $v$ and $x$ be points such that there is a partial cogeodesic $((u, \ldots, v),(x))$ for a point $u \in\langle y, z\rangle_{\mathrm{g}}$. Then $\min \left\{\operatorname{cod}(w, x) \mid w \in\langle y, z\rangle_{\mathrm{g}}\right\}=\min \left\{\operatorname{cod}(w, x) \mid w \in\langle y, z, v\rangle_{\mathrm{g}}\right\}$.

Proof. Set $U:=\langle y, z\rangle_{\mathrm{g}}$. Let $s$ be a geodesic from $u \in U$ to $v$ passing $\operatorname{pr}_{U}(v)$ and let $s^{\prime}$ be the geodesic from $\operatorname{pr}_{U}(v)$ to $v$ contained in $s$. Since $((u, \ldots, v),(x))$ is a partial cogeodesic, $(s,(x))$ is a partial cogeodesic and $\left(s^{\prime},(x)\right)$ is one, too. Hence we may assume $u=\operatorname{pr}_{U}(v)$. We set $k:=\mathrm{d}(v, u)$ and $\left(v_{i}\right)_{0<i<k}:=s$, where $v_{0}=u$. Further set $l:=\mathrm{d}\left(u, \operatorname{copr}_{U}(x)\right)$ and let $\left(u_{i}\right)_{0 \leq i \in l}$ be a geodesic from $u$ to $\operatorname{copr}_{U}(x)$.

Now we recursively define points $w_{i}$ for $0 \leq i \leq l$ with $w_{i} \sim u_{i}, w_{i} \notin U$ and $\operatorname{cod}\left(w_{i}, x\right)=\operatorname{cod}\left(u_{i}, x\right)+1$. Set $w_{0}:=v_{1}$. Now let $i<l$ such that $w_{i}$ is defined. Then $\left\langle w_{i}, u_{i+1}\right\rangle_{\mathrm{g}} \leq\left\langle w_{i}, U\right\rangle_{\mathrm{g}}$. Since $w_{i} \notin U$ and $w_{i} \sim u_{i}$, we obtain $u_{i}=\operatorname{pr}_{U}\left(w_{i}\right)$ and thus $\mathrm{d}\left(w_{i}, u_{i+1}\right)=2$ by Proposition 3.16. Set $w_{i+1}:=\operatorname{copr}_{\left\langle w_{i}, u_{i+1}\right\rangle_{\mathrm{g}}}(x)$. With Lemma 3.5 we obtain $\operatorname{cod}\left(w_{i+1}, x\right)=\operatorname{cod}\left(w_{i}, x\right)+1$ since $\operatorname{cod}\left(u_{i+1}, x\right)=\operatorname{cod}\left(u_{i}, x\right)+$ $1=\operatorname{cod}\left(w_{i}, x\right)$. Now $w_{i} \notin U$ implies $\left\langle w_{i}, u_{i+1}\right\rangle_{\mathrm{g}} \cap U=u_{i} u_{i+1}$ and hence $w_{i+1} \notin U$ since $\operatorname{cod}\left(x, u_{i} u_{i+1}\right)<\operatorname{cod}\left(w_{i+1}, x\right)$. Furthermore, $w_{i+1} \sim u_{i+1}$ by Proposition 3.13.

${ }^{2}$ We merge partial cogeodesics in the canonical way. 
With $\left\langle u_{i}, w_{i+1}\right\rangle_{\mathrm{g}}=\left\langle w_{i}, u_{i+1}\right\rangle_{\mathrm{g}}$ we obtain $\left\langle w_{i}, U\right\rangle_{\mathrm{g}}=\left\langle w_{i+1}, U\right\rangle_{\mathrm{g}}$ and consequently $\left\langle v_{1}, U\right\rangle_{\mathrm{g}}=\left\langle w_{l}, U\right\rangle_{\mathrm{g}}$. Lemma 4.3 implies $\operatorname{diam}\left(\left\langle v_{1}, U\right\rangle_{\mathrm{g}}\right)=\operatorname{diam}(U)+1$. Together with $u_{l}=\operatorname{copr}_{U}(x)$ and $\operatorname{cod}\left(w_{l}, x\right)=\operatorname{cod}\left(u_{l}, x\right)+1$ we obtain $w_{l}=\operatorname{copr}_{\left\langle v_{1}, U\right\rangle_{\mathrm{g}}}(x)$ and therefore $\min \{\operatorname{cod}(u, x) \mid u \in U\}=\min \left\{\operatorname{cod}(u, x) \mid u \in\left\langle v_{1}, U\right\rangle_{\mathrm{g}}\right\}$ by Lemma 3.5. Since $u \in\left\langle v_{1}, U\right\rangle_{\mathrm{g}}$, the claim follows by repeating this procedure $k$ times.

Lemma 5.7. Let $U$ and $V$ be convex subspaces of an $L D P$ with $\operatorname{diam}(U) \leq \operatorname{diam}(V)=$ $n$ such that $U$ is coparallel to $V$. For a pair $(x, y) \in U \times V$ and a point $z$ with $\operatorname{cod}(x, z)=$ $\operatorname{cod}(x, y)+\mathrm{d}(y, z)<\infty$, the subspace $U$ is coparallel to $\langle z, V\rangle_{\mathrm{g}}$ with $\min \{\operatorname{cod}(u, v) \mid$ $(u, v) \in U \times V\}=\min \left\{\operatorname{cod}(u, v) \mid(u, v) \in U \times\langle z, V\rangle_{\mathrm{g}}\right\}$.

Proof. Set $m:=\operatorname{cod}(U, V)$. Let $\left(z_{i}\right)_{0 \leq i \leq k}$ be a geodesic where $k=\mathrm{d}(z, V), z_{0}=$ $\operatorname{pr}_{V}(z)$ and $z_{k}=z$. Then $\left\langle z_{i+1}, V\right\rangle_{\mathrm{g}}=\left\langle z_{i+1},\left\langle z_{i}, V\right\rangle_{\mathrm{g}}\right\rangle_{\mathrm{g}}$ and $\operatorname{cod}\left(x, z_{i+1}\right)=\operatorname{cod}(x, y)+$ $\mathrm{d}\left(y, z_{i+1}\right)$ for every $i<k$. Therefore the claim holds if and only if it holds for $k=1$. Hence, let $k=1$ and set $V^{\prime}:=\langle z, V\rangle_{\mathrm{g}}$.

By Lemma 4.3 we obtain $\operatorname{diam}\left(V^{\prime}\right)=n+1$. Furthermore, $\min \{\operatorname{cod}(x, v) \mid v \in$ $V\}=\min \left\{\operatorname{cod}(x, v) \mid v \in V^{\prime}\right\}=m-n$ by Lemma 5.6 and therefore $\operatorname{cod}\left(x, V^{\prime}\right)=$ $m+1$ by Lemma 3.5. Set $x^{\prime}:=\operatorname{copr}_{V}(x)$ and $w:=\operatorname{copr}_{V^{\prime}}(x)$. Then $\operatorname{cod}(x, w)=m+1$ and $w \sim x^{\prime}$. Thus, $x=\operatorname{copr}_{U}(w)$ since $x=\operatorname{copr}_{U}\left(x^{\prime}\right)$ and $\operatorname{cod}\left(x, x^{\prime}\right)=m$. Let $u \in U$ and set $v:=\operatorname{copr}_{V}(u)$. Then $\operatorname{cod}(w, u)=m+1-\mathrm{d}(u, x)$ and $\mathrm{d}(w, v)=\mathrm{d}\left(x^{\prime}, v\right)+1=$ $\mathrm{d}(u, x)+1$ by Propositions 3.13 and 3.16. Hence, $v$ is not a cogate for $u$ in $\langle v, w\rangle_{\mathrm{g}}$. This implies $\operatorname{cod}\left(u, V^{\prime}\right) \geq m+1$. By Lemma 3.5 we conclude $\min \{\operatorname{cod}(u, p) \mid p \in$ $V\}=m-n$ and consequently again by Lemma $3.5 \operatorname{cod}\left(u, \operatorname{copr}_{V^{\prime}}(u)\right)=m+1$ and $\min \left\{\operatorname{cod}(u, p) \mid p \in V^{\prime}\right\}=m-n$.

Let $U$ be a point-line space. Then for a point $x \in U$ we denote by ${ }_{x} U$ the connected component of $U$ that contains $x$.

Lemma 5.8. Let $x$ and $y$ be points of an $A D P \mathscr{S}$ with $\operatorname{cod}(x, y)=n$ and $\mathrm{d}(x, y)=\infty$. Then $\left\langle\langle x, y\rangle_{\mathrm{g}}={ }_{x}\left\langle\langle x, y\rangle_{\mathrm{g}} \cup_{y}\left\langle\langle x, y\rangle_{\mathrm{g}}\right.\right.\right.$ and ${ }_{x}\langle x, y\rangle_{\mathrm{g}}=\bigcup\left\{\langle x, z\rangle_{\mathrm{g}} \mid z \in{ }_{x} \mathscr{S} \wedge \operatorname{cod}(x, y)+\right.$ $\mathrm{d}(z, x)=\operatorname{cod}(z, y)\}$.

Proof. Set $U:=\bigcup\left\{\langle x, z\rangle_{\mathrm{g}} \mid z \in{ }_{x} \mathscr{S} \wedge \operatorname{cod}(x, y)+\mathrm{d}(z, x)=\operatorname{cod}(z, y)\right\}$ and $V:=$ $\bigcup\left\{\langle y, z\rangle_{\mathrm{g}} \mid z \in{ }_{y} \mathscr{S} \wedge \operatorname{cod}(x, y)+\mathrm{d}(z, y)=\operatorname{cod}(z, x)\right\}$. Now let $z \in{ }_{x} \mathscr{S}$ be a point with $\operatorname{cod}(x, y)+\mathrm{d}(z, x)=\operatorname{cod}(z, y)$ and let $\left(x_{i}\right)_{0 \leq i \leq n}$ be a geodesic from $x$ to $z$. Then $\left(\left(x_{i}\right)_{0 \leq i \leq n},(y)\right)$ is a partial cogeodesic and hence $\langle x, z\rangle_{\mathrm{g}} \leq\left\langle\langle x, y\rangle_{\mathrm{g}}\right.$. Thus, $U \subseteq{ }_{x}\left\langle\langle x, y\rangle_{\mathrm{g}}\right.$ and analogously $V \subseteq{ }_{y}\langle x, y\rangle_{\mathrm{g}}$. Hence, it suffices to show that $U \cup V$ is a coconvex subspace.

For $i \in\{0,1\}$ let $z_{i} \in{ }_{x} \mathscr{S}$ be a point with $\operatorname{cod}(x, y)+\mathrm{d}\left(z_{i}, x\right)=\operatorname{cod}\left(z_{i}, y\right)$. Then $\min \left\{\operatorname{cod}(p, y) \mid p \in\left\langle x, z_{0}, z_{1}\right\rangle_{\mathrm{g}}\right\}=\min \left\{\operatorname{cod}(p, y) \mid p \in\left\langle x, z_{0}\right\rangle_{\mathrm{g}}\right\}=\operatorname{cod}(x, y)$ by Lemma 5.6. Set $z_{2}:=\operatorname{copr}_{\left\langle x, z_{0}, z_{1}\right\rangle_{\mathrm{g}}}(y)$. Then $\operatorname{cod}(x, y)+\mathrm{d}\left(z_{2}, x\right)=\operatorname{cod}\left(z_{2}, y\right)$ by Proposition 3.13. This implies $\left\langle x, z_{0}, z_{1}\right\rangle_{\mathrm{g}}=\left\langle x, z_{2}\right\rangle_{\mathrm{g}} \leq U$ and thus, $U$ is a convex subspace. Analogously, $V$ is a convex subspace and therefore $U \cup V$ is a convex subspace.

For symmetric reasons it remains now to show that for $u \in U$ and $v \in V$ every point $w$ with $w \sim u$ and $\operatorname{cod}(w, v)=\operatorname{cod}(u, v)+1$ is contained in $U$. Let $z_{u} \in U$ and $z_{v} \in V$ 
be points with $\operatorname{cod}(x, y)=\operatorname{cod}\left(z_{u}, y\right)-\mathrm{d}\left(z_{u}, x\right)=\operatorname{cod}\left(z_{v}, x\right)-\mathrm{d}\left(z_{v}, y\right)$, such that $u \in\left\langle x, z_{u}\right\rangle_{\mathrm{g}}$ and $v \in\left\langle y, z_{v}\right\rangle_{\mathrm{g}}$. By Definition 3.4 there is a point $w_{v}$ at distance $\mathrm{d}\left(y, z_{v}\right)$ to $x$, such that $\left\langle x, w_{v}\right\rangle_{\mathrm{g}}$ is coparallel to $\left\langle y, z_{v}\right\rangle_{\mathrm{g}}$ and $\operatorname{cod}(x, y)=\min \{\operatorname{cod}(p, y) \mid p \in$ $\left.\left\langle x, w_{v}\right\rangle_{\mathrm{g}}\right\}$. We use Lemma 5.7 twice to conclude that $\left\langle y, z_{v}\right\rangle_{\mathrm{g}}$ is coparallel to $\left\langle x, w_{v}, z_{u}\right\rangle_{\mathrm{g}}$ and $\left\langle x, w_{v}, z_{u}, w\right\rangle_{\mathrm{g}}=: U^{\prime}$ with $\min \left\{\operatorname{cod}(p, y) \mid p \in U^{\prime}\right\}=\operatorname{cod}(x, y)$. Hence, $U^{\prime}=$ $\left\langle x, \operatorname{copr}_{U^{\prime}}(y)\right\rangle_{\mathrm{g}}$ and since $\operatorname{copr}_{U^{\prime}}(y)$ lies on a cogeodesic from $x$ to $y$, we obtain $w \in$ $U^{\prime} \leq U$.

Analogously to the diameter we define the codiameter for a set of points $M$ as $\operatorname{codm}(M):=\min \left\{\operatorname{cod}(x, y) \mid(x, y) \in M^{2}\right\}$.

Proposition 5.9. Let $\mathscr{S}$ be an $A D P$ and let $x$ and $y$ be two points of $\mathscr{S}$ at codistance $n$. Then $\operatorname{codm}\left(\left\langle\langle x, y\rangle_{\mathrm{g}}\right)=n\right.$ and for every point $u \in\left\langle\langle x, y\rangle_{\mathrm{g}}\right.$ there is a point $v \in\left\langle\langle x, y\rangle_{\mathrm{g}}\right.$ at codistance $n$. Furthermore, $\left\langle\langle x, y\rangle_{\mathrm{g}}=\left\langle\langle u, v\rangle_{\mathrm{g}}\right.\right.$.

Proof. If $\mathrm{d}(x, y)<\infty$, namely $\mathscr{S}$ has finite diameter, then all claims are clear. Hence we may assume $\mathrm{d}(x, y)=\infty$.

Set $W:=\left\langle\langle x, y\rangle_{\mathrm{g}}\right.$ and let $u \in{ }_{x} W$. Then there is a point $z \in{ }_{x} W$ such that $u \in$ $\langle x, z\rangle_{\mathrm{g}}=: U$ and $\operatorname{cod}(y, z)-\mathrm{d}(z, x)=n$. By Definition 3.4 there is a point $v^{\prime}$ such that $V:=\left\langle v^{\prime}, y\right\rangle_{\mathrm{g}}$ is coparallel to $U$ with $\operatorname{cod}(U, V)=n+\mathrm{d}(x, z)$. Thus, we obtain $V=\left\langle\operatorname{copr}_{V}(x), y\right\rangle_{\mathrm{g}}$ and therefore $V \leq W$. Since $U$ is coparallel to $V$, there is a point $u^{\prime} \in V$ with $\operatorname{cod}\left(u, u^{\prime}\right)=n$.

Now let $v \in W$ be an arbitrary point with $\operatorname{cod}(u, v)=n$. Then there is a point $z^{\prime} \in$ ${ }_{y} W$ such that $v \in\left\langle y, z^{\prime}\right\rangle_{\mathrm{g}}$ and $\operatorname{cod}\left(x, z^{\prime}\right)-\mathrm{d}\left(z^{\prime}, y\right)=n$. Then $U$ is coparallel to $\left\langle z^{\prime}, V\right\rangle_{\mathrm{g}}$ and $\min \left\{\operatorname{cod}(u, p) \mid p \in\left\langle z^{\prime}, V\right\rangle_{\mathrm{g}}\right\}=n$ by Lemma 5.7. Thus, $U=\left\langle u, \operatorname{copr}_{U}(v)\right\rangle_{\mathrm{g}}$ and $\left\langle z^{\prime}, V\right\rangle_{\mathrm{g}}=\left\langle v, \operatorname{copr}_{\left\langle z^{\prime}, V\right\rangle_{\mathrm{g}}}(u)\right\rangle_{\mathrm{g}}$. This implies $U \cup\left\langle z^{\prime}, V\right\rangle_{\mathrm{g}} \subseteq\langle u, v\rangle_{\mathrm{g}}$ and therefore $\langle u, v\rangle_{\mathrm{g}}=\left\langle\langle x, y\rangle_{\mathrm{g}}\right.$.

Finally, $\operatorname{cod}(u, p) \geq n$ and $\operatorname{cod}(p, v) \geq n$ for all $p \in W$ follows by Lemma 5.8.

We introduced gates only for connected subspaces, see Definition 3.12. Now we expand this definition to the case where the subspace is disconnected.

Definition 5.10. Let $U$ be a subspace of an LDP $\mathscr{S}$ and let $p$ be a point at finite distance to $U$. Set $U_{0}:={ }_{p} \mathscr{S} \cap U$. If there is a point $q \in U_{0}$ such that $q$ is the gate for $p$ in $U_{0}$ and for every point $r \in U \backslash U_{0}$ there is a cogeodesic from $r$ to $p$ such that the path containing $p$ also contains $q$, we call $q$ the gate of $p$ in $U$. If for every point $r \in \mathcal{P}$ with $\mathrm{d}(r, U)<\infty$ the gate of $r$ in $U$ exists, we call $U$ gated.

Proposition 5.11. In an ADP the coconvex span of two points at finite codistance is gated.

Proof. Let $x$ and $y$ be points of an ADP $\mathscr{S}$ with $\operatorname{cod}(x, y)<\infty$. By Proposition 3.16 we may restrain to the case $\operatorname{diam}(\mathscr{S})=\infty$ and hence $\mathrm{d}(x, y)=\infty$. Set $U:=\left\langle\langle x, y\rangle_{\mathrm{g}}\right.$ and let $z$ be a point of $\mathscr{S}$. Since $\mathscr{S}={ }_{x} \mathscr{S} \cup{ }_{y} \mathscr{S}$, we may assume $z \in{ }_{y} \mathscr{S}$ and hence $\mathrm{d}(y, z)<\infty$. Let $z_{0}$ and $z_{1}$ be points in ${ }_{y} U$ at distance $\mathrm{d}(z, U)$ to $z$. Since there is a gate in $\left\langle y, z_{0}, z_{1}\right\rangle_{\mathrm{g}} \leq{ }_{y} U$ for $z$ by Proposition 3.16 we obtain $z_{0}=z_{1}=\operatorname{pr}_{U}(z)=: z^{\prime}$. For 
every point $p \in{ }_{y} U$, the point $z^{\prime}$ is a gate for $z$ in $\left\langle p, z^{\prime}\right\rangle_{\mathrm{g}} \leq U$ and therefore $z^{\prime}$ is a gate for $z$ in ${ }_{y} U$.

Now let $w \in{ }_{x} U$ and set $w^{\prime}:=\operatorname{copr}_{\left\langle z, z^{\prime}\right\rangle_{\mathrm{g}}}(w)$. Then $\operatorname{cod}\left(w^{\prime}, w\right)=\operatorname{cod}\left(z^{\prime}, w\right)+$ $\mathrm{d}\left(w^{\prime}, z^{\prime}\right)$ by Proposition 3.13. Hence, $w^{\prime} \in\left\langle\left\langle w, z^{\prime}\right\rangle_{\mathrm{g}} \leq U\right.$ and therefore $\mathrm{d}\left(w^{\prime}, z\right)=$ $\mathrm{d}\left(w^{\prime}, z^{\prime}\right)+\mathrm{d}\left(z^{\prime}, z\right)$. Thus, $\operatorname{cod}\left(w, z^{\prime}\right)=\operatorname{cod}\left(w, w^{\prime}\right)-\mathrm{d}\left(w^{\prime}, z^{\prime}\right)=\operatorname{cod}\left(w, w^{\prime}\right)-$ $\mathrm{d}\left(w^{\prime}, z\right)+\mathrm{d}\left(z^{\prime}, z\right)=\operatorname{cod}(w, z)+\mathrm{d}\left(z^{\prime}, z\right)$. Hence, $z^{\prime}$ is a gate for $z$ in $U$ and $U$ is gated.

Corollary 5.12. Let $U$ be a coconvex subspace of an $A D P$ such that $\operatorname{codm}(U)<\infty$. Then $U$ is the coconvex span of two points.

Proof. Set $n:=\operatorname{codm}(U)$ and let $x$ and $y$ be two points of $U$ with $\operatorname{cod}(x, y)=n$. Let $z \in U$ and set $z^{\prime}:=\operatorname{pr}_{\langle x, y\rangle_{\mathrm{g}}}(z)$. Then there is a point $w \in\left\langle\langle x, y\rangle_{\mathrm{g}}\right.$ with $\operatorname{cod}\left(w, z^{\prime}\right)=n$. Since $\operatorname{cod}(w, z)=n-\mathrm{d}\left(z, z^{\prime}\right)$ by Proposition 5.11, we obtain $z=z^{\prime}$ with $\operatorname{codm}(U)=$ $n$.

Lemma 5.13. Let $U$ be a coconvex subspace of an $A D P$ with $\operatorname{codm}(U)<\infty$ and let $x$ be a point with $\mathrm{d}(x, U)=n<\infty$. Then $\operatorname{codm}\left(\langle x, U\rangle_{\mathrm{g}}\right)=\operatorname{codm}(U)-n$.

Proof. Set $x^{\prime}:=\operatorname{pr}_{U}(x)$ and let $y \in U$ be a point with $\operatorname{cod}\left(x^{\prime}, y\right)=\operatorname{codm}(U)$. Then $x^{\prime}$ is on a cogeodesic from $x$ to $y$. Thus, $\left\langle\left\langle x^{\prime}, y\right\rangle_{\mathrm{g}} \leq\left\langle\langle x, y\rangle_{\mathrm{g}}\right.\right.$ and therefore $\left\langle\langle x, U\rangle_{\mathrm{g}}=\left\langle\langle x, y\rangle_{\mathrm{g}}\right.\right.$. The claim follows by Proposition 5.11.

Corollary 5.14. Let $U$ be a coconvex subspace of an $A D P$. Then $U=\mathscr{S}$ if and only if $\operatorname{codm}(U)=0$.

Proof. This is an immediate consequence of Lemma 5.13.

In an $\mathrm{ADP} \mathscr{S}$ we denote by $\mathcal{U}^{k}(\mathscr{S})$ for $k \in \mathbb{N}$ the set of coconvex subspaces with codiameter $k$. If it is clear that we refer to $\mathscr{S}$, we rather write $\mathcal{U}^{k}$. Note that for $\operatorname{diam}(\mathscr{S})<\infty$ this notation coincides with the one that we already introduced.

Lemma 5.15. Let $U \in \mathcal{U}^{n}$ be a subspace of an $A D P$, where $n \in \mathbb{N}$. Further let $H$ and $V$ be two coconvex subspaces of $U$ where $\operatorname{codm}(H)=n+1$ and $\operatorname{cod} \operatorname{m}(V)<\infty$. Then $V \leq H$ or $V \cap H=\emptyset$ or $V \cap H \in \mathcal{U}^{\operatorname{codm}(V)+1}$.

Proof. Let $V \cap H \neq \emptyset$ and $V \not L H$. By Proposition 5.9 we may assume $V=\left\langle\langle u, v\rangle_{\mathrm{g}}\right.$ where $u \in H$ and $v \notin H$. By Proposition $5.11 v$ has a gate $v^{\prime}$ in $H$. We obtain $v^{\prime} \in$ $\langle u, v\rangle_{\mathrm{g}}=V$. Let $w \in H$ be a point with $\operatorname{cod}\left(w, v^{\prime}\right)=n+1$. Since $v \notin H$, Lemma 5.13 implies $\mathrm{d}\left(v, v^{\prime}\right)=1$. Thus, $\operatorname{codm}(V \cap H) \geq \mathrm{d}\left(u, v^{\prime}\right)=\operatorname{codm}(V)-1$. Since $V \cap$ $H$ is coconvex and $V \cap H \neq V$, we conclude $\operatorname{codm}(V \cap H)=\operatorname{codm}(V)-1$ by Proposition 5.9.

Lemma 5.16. Let $U \in \mathcal{U}^{n}$ be a subspace of an $A D P$, where $n \in \mathbb{N}$. Further let $H \leq U$ be a subspace with $H \in \mathcal{U}^{n+1}$. Then for every point $p \in U \backslash H$ there is a subspace $J \in \mathcal{U}^{n+1}$ with $p \in J \leq U$ and $H \cap J=\emptyset$. 
Proof. Set $p^{\prime}:=\operatorname{pr}_{H}(p)$. We choose a point $q \in U$ with $\operatorname{cod}\left(p^{\prime}, q\right)=n$ and set $q^{\prime}:=$ $\operatorname{pr}_{H}(q)$. Since $\operatorname{codm}(H)=n+1$ we obtain $q \notin U$ and therefore $p \sim p^{\prime}$ and $q \sim q^{\prime}$ by Lemma 5.13. By Proposition 5.11 this implies that $p^{\prime}$ is the gate for $p$ and $q^{\prime}$ is the gate for $q$ in $H$. Thus, $\operatorname{cod}\left(p^{\prime}, q^{\prime}\right)=n+1$ and consequently $\operatorname{cod}\left(p, q^{\prime}\right)=n$. Set $r:=\operatorname{copr}_{q q^{\prime}}(p)$. Since $\operatorname{cod}\left(p, q^{\prime}\right)=n$ and $\operatorname{codm}(U)=n$ we obtain $\operatorname{cod}(p, r)=n+1$. Thus, $r \neq q^{\prime}$ and we conclude $r \notin H$ and $q^{\prime}=\operatorname{pr}_{H}(r)$. Set $J:=\langle p, r\rangle_{\mathrm{g}}$. Assume there is a point $s \in H \cap{ }_{p} J$. Then $p^{\prime} \in\langle p, s\rangle_{\mathrm{g}} \leq J$ and $q^{\prime} \in\left\langle\langle r, s\rangle_{\mathrm{g}} \leq J\right.$ by Proposition 5.11. Hence, $J=\left\langle p^{\prime}, q^{\prime}\right\rangle_{\mathrm{g}}=H$ by Proposition 3.11, a contradiction to $p \in J \backslash H$. Analogously, $H \cap{ }_{r} J=\emptyset$.

Proposition 5.17. Let $U \neq \emptyset$ be a coconvex subspace of an $A D P$ with $\operatorname{codm}(U)<\infty$. Then $U$ is an $A D P$.

Proof. Set $n:=\operatorname{codm}(U)$ and $R:=\{(x, y) \in U \times U \mid \operatorname{cod}(x, y)=n\}$. Then $R$ is symmetric and total by Proposition 5.9. Now let $x$ and $y$ be points in $U$ with $\operatorname{cod}(x, y)=$ $k$. Let $x^{\prime}$ be a point with $x \leftrightarrow x^{\prime}$ and $\mathrm{d}\left(y, x^{\prime}\right)=k$. Then $\mathrm{d}\left(x^{\prime}, U\right)=n$ by Lemma 5.13. This implies $\left(x, \operatorname{pr}_{U}\left(x^{\prime}\right)\right) \in R$ and hence $\operatorname{cod}_{R}(x, y)=k-n$ since $\mathrm{d}\left(y, \operatorname{pr}_{U}\left(x^{\prime}\right)\right)=$ $\mathrm{d}\left(y, x^{\prime}\right)-\mathrm{d}\left(\operatorname{pr}_{U}\left(x^{\prime}\right), x^{\prime}\right)$.

Now let $z \in{ }_{y} U$. Then there is a point $w$ such that $X:=\langle w, x\rangle_{\mathrm{g}}$ is coparallel to $Y:=\langle y, z\rangle_{\mathrm{g}}$. Set $y^{\prime}:=\operatorname{copr}_{Y}(w)$. Then $\operatorname{cod}\left(x, y^{\prime}\right)=\operatorname{cod}\left(w, y^{\prime}\right)-\mathrm{d}(w, x)$ and therefore $w \in\left\langle\left\langle x, y^{\prime}\right\rangle_{\mathrm{g}} \leq U\right.$. Hence, $X \leq U$ and $X$ is $R$-coparallel to $Y$ with $\operatorname{cod}_{R}(X, Y)=$ $\operatorname{cod}(X, Y)-n=\min \{\operatorname{cod}(x, p) \mid p \in Y\}+\mathrm{d}(y, z)-n=\min \left\{\operatorname{cod}_{R}(x, p) \mid p \in\right.$ $Y\}+\mathrm{d}(y, z)$.

Definition 5.18. Let $\mathscr{S}$ be an ADP. For every subspace $G \in \mathcal{U}^{2}$ set $[G]:=\left\{P \in \mathcal{U}^{1} \mid\right.$ $G<P\}$. We call $\mathscr{D}(\mathscr{S}):=\left(\mathcal{U}^{1},\left\{[G] \mid \emptyset \neq G \in \mathcal{U}^{2}\right\}\right)$ the dual of $\mathscr{S}$.

This definition is a generalisation of Definition 4.7. As in the case of LDPs with finite diameter we will write $P \perp Q$ for $\{P, Q\} \subseteq \mathcal{U}^{1}$, if $P=Q$ or $\emptyset \neq P \cap Q \in \mathcal{U}^{2}$. By Lemma 5.15 we obtain $P \perp Q$ whenever $P \cap Q \neq \emptyset$.

Theorem 5.19. The dual of an ADP with diameter $\geq 1$ is a non-degenerate polar space.

Proof. For ADPs of finite diameter the claim coincides with Theorem 4.8. For ADPs of infinite diameter the proof is analogous to the one for Theorem 4.8. Since for ADPs of infinite diameter the elements of $\mathcal{U}^{1}$ and $\mathcal{U}^{2}$ are coconvex subspaces, we have to use Lemma 5.13 instead of Lemma 4.3 and Lemma 5.16 instead of Lemma 4.5.

Lemma 5.20. Let $\mathscr{S}$ be an $A D P$ and let $p$ be a point of $\mathscr{S}$. Then $M_{p}:=\left\{P \in \mathcal{U}^{1} \mid p \in\right.$ $P\}$ is a generator of $\mathscr{D}(\mathscr{S})$.

Proof. For two distinct elements $P$ and $Q$ of $M_{p}$, we obtain $p \in P \cap Q \in \mathcal{U}^{2}$ by Lemma 5.15. Hence, there is line in $\mathscr{D}(\mathscr{S})$ joining $P$ and $Q$ and all points on this line are elements of $M_{p}$. Thus, $M_{p}$ is a singular subspace. Now let $R \in \mathcal{U}^{1} \backslash M_{p}$. Then by Lemma 5.16 there is an coconvex subspace $P \in M_{p}$ being disjoint to $R$. Hence, $M_{p}$ is a generator of $\mathscr{D}(\mathscr{S})$. 
The following theorem is the main difference between ADPs of finite diameter and ADPs with infinite diameter. While the ones with finite diameter are dual polar spaces, the ones with infinite diameter are always proper subspaces of dual polar spaces.

Theorem 5.21. Every ADP with infinite diameter consists of exactly two connected components of a dual polar space.

Proof. Let $\mathscr{S}$ denote an ADP with infinite diameter. We show that the dual polar space of $\mathscr{D}(\mathscr{S})$, denoted by $\overline{\mathscr{S}}$, contains two connected components $X$ and $Y$ such that $\mathscr{S}$ is isomorphic to the subspace $X \cup Y$.

Let $M$ be a generator of $\mathscr{D}(\mathscr{S})$. We show that the intersection of the elements of $M$ is either empty or consists of a single point. In the latter case $M$ consists of all elements of $\mathcal{U}^{1}$ containing this single point. Set $S:=\bigcap_{P \in M} P$. By construction $S \leq \mathscr{S}$ is coconvex. Suppose $S$ contains a line $l$ and let $p \in l$. Further let $q \in \mathscr{S}$ be a point with $p \leftrightarrow q$ and set $r:=\operatorname{copr}_{l}(q)$. Then $Q:=\left\langle\langle r, q\rangle_{\mathrm{g}} \in \mathcal{U}^{1}\right.$ and $P \cap Q \neq \emptyset$ for every $P \in M$. Hence, there is a singular subspace of $\mathscr{D}(\mathscr{S})$ containing $M$ and $Q$. Since $M$ is a generator, this implies $Q \leq M$. This is a contradiction since $x \leftrightarrow y$ and therefore $x \notin Q$. Now suppose that $S$ contains two distinct points $p$ and $q$. Then $\mathrm{d}(p, q)=\infty$ since otherwise $\langle p, q\rangle_{\mathrm{g}} \leq S$ and hence $S$ would contain a line. Thus, $\operatorname{cod}(p, q)<\infty$. Since $\operatorname{cod}\left(p,{ }_{q} \mathscr{S}\right)=\infty$ there is a point $q^{\prime} \in{ }_{q} \mathscr{S}$ with $\operatorname{cod}\left(p, q^{\prime}\right)>\operatorname{cod}(p, q)$ and $p^{\prime}:=\operatorname{pr}_{\left\langle q, q^{\prime}\right\rangle_{\mathrm{g}}}(p)$ is on a cogeodesic from $p$ to $q$. Hence, $p^{\prime} \in\left\langle\langle p, q\rangle_{\mathrm{g}} \leq S\right.$, a contradiction since $\mathrm{d}\left(p^{\prime}, q\right)<\infty$. Thus, $S$ contains at most one point.

Conversely, $M_{p}:=\left\{P \in \mathcal{U}^{1} \mid p \in P\right\}$ is a generator of $\mathscr{D}(\mathscr{S})$ by Lemma 5.20. Therefore, mapping $p$ to $M_{p}$ yields an injection from the points of $\mathscr{S}$ in the set of generators of $\mathscr{D}(\mathscr{S})$. We will show that this injection is a morphism.

Since in an LDP and in a dual polar space every set of pairwise collinear points is contained in a line, it suffices to show that for two points $p$ and $q$ of $\mathscr{S}$ the generators $M_{p}$ and $M_{q}$ contain a common hyperplane if and only if $p \sim q$. First assume $p \nsim q$. Since $\{p\}=\bigcap_{Q \in M_{p}} Q$, there is an element $P \in M_{p}$ with $q \notin P$. Then $q \sim q^{\prime}:=\operatorname{pr}_{P}(q)$ by Lemma 5.13 and hence $p \neq q^{\prime}$. By Proposition 5.17P is an ADP of infinite diameter and therefore $\bigcap\left\{L \in \mathcal{U}^{1}(P) \mid p \in L\right\}=\{p\}$. Hence, there is an element $G \in \mathcal{U}^{2}$ with $p \in G<P$ and $q^{\prime} \notin G$. This implies $\mathrm{d}(q, G)=\mathrm{d}\left(q, q^{\prime}\right)+\mathrm{d}\left(q^{\prime}, G\right) \geq 2$. Thus, $\langle q, G\rangle_{\mathrm{g}}=\mathscr{S}$ by Lemma 5.13 and therefore $[G] \cap M_{q}=\emptyset$. Hence, $M_{p} \cap M_{q}$ is no hyperplane of $M_{p}$ since $[G] \leq M_{p}$. Now assume $p \sim q$ and let $G \in \mathcal{U}^{2}$ with $[G] \leq M_{p}$. If $q \in G$, we obtain $[G] \leq M_{q}$. If $q \notin G$, we obtain $\langle q, G\rangle_{\mathrm{g}} \in \mathcal{U}^{1}$ by Lemma 5.13. Hence, $[G] \cap M_{q} \neq \emptyset$ and therefore $M_{p}$ and $M_{q}$ have a hyperplane in common.

We prove now that $\left\{M_{p} \mid p \in \mathscr{S}\right\}$ is the union of two connected components of $\overline{\mathscr{S}}$. Since $p \sim q \Leftrightarrow M_{p} \sim M_{q}$ for two points $p$ and $q$ of $\mathscr{S}$, we conclude that $p$ and $q$ are connected if and only if $M_{p}$ and $M_{q}$ are connected in $\overline{\mathscr{S}}$. Hence, $\left\{M_{p} \mid p \in \mathscr{S}\right\}$ consists of two connected components and it remains to show that they are also connected components of $\overline{\mathscr{S}}$. Let $p \in \mathscr{S}$ and let $M \neq M_{p}$ be a generator of $\mathscr{D}(\mathscr{S})$ such that $M \sim$ $M_{p}$. Set $H:=M \cap M_{p}$ and let $Q \in M \backslash H$. Then $p \notin Q$ and hence, $p \sim q:=\operatorname{pr}_{Q}(p)$ by Lemma 5.13. Now let $P \in H$. Then there is a point $r \in P \cap Q$ and we obtain $q \in\langle p, r\rangle_{\mathrm{g}} \leq P$. With $H \leq M_{q}$ and $Q \in M_{q}$ we conclude $\langle Q, H\rangle \leq M_{q}$. Since $Q \notin H$ and $H$ is a hyperplane of $M$ this implies $M=M_{q}$. Hence, ${ }_{M_{p}} \overline{\mathscr{S}} \leq\left\{M_{x} \mid x \in \mathscr{S}\right\}$ and 
therefore $\mathscr{S}$ is isomorphic to the union of two connected components of the dual polar space $\overline{\mathscr{S}}$.

The following proposition shows why an ADP of infinite diameter can never be a dual polar space.

Proposition 5.22. A dual polar space is connected if and only if it has finite rank. Moreover, a dual polar space of infinite rank has more than two connected components.

Proof. We consider the underlying non-degenerate polar space $\mathscr{S}$ of a dual polar space. If $\mathscr{S}$ has finite rank, then $\operatorname{crk}_{M}(M \cap N)<\infty$ for every two generators $M$ and $N$ of $\mathscr{S}$. Hence, the dual of $\mathscr{S}$ is connected by Proposition 2.10.

Now let $\mathscr{S}$ be of infinite rank. Then by Proposition 2.13 there are two generators $M$ and $N$ with $\mathrm{d}^{*}(M, N)=\infty$. Let $\mathcal{M}$ be the set of pairs $(X, Y, \varphi)$ such that $X \subseteq M$ and $Y \subseteq N$ are independent sets of points with $\langle X\rangle \cap N=\emptyset$ and $\langle Y\rangle \cap M=\emptyset$ such that $X \subseteq Y^{\perp}$ and $\varphi$ is a bijection from $X$ to $Y$. Further let $\prec$ be a strict partial order on $\mathcal{M}$ with $(X, Y, \varphi) \prec\left(X^{\prime}, Y^{\prime}, \varphi^{\prime}\right) \Leftrightarrow\left(X<X^{\prime} \wedge Y<\left.Y^{\prime} \wedge \varphi^{\prime}\right|_{X}=\varphi\right)$. Now let $\left(X_{i}, Y_{i}, \varphi_{i}\right)_{i \in I}$ be a chain in $\mathcal{M}$ with respect to $\prec$ for an index set $I$. Then $X:=\bigcup_{i \in I} X_{i}$ is again an independent set of points with $\langle X\rangle \cap N=\emptyset$. Analogously, $Y:=\bigcup_{i \in I} Y_{i}$ is independent with $\langle Y\rangle \cap M=\emptyset$. Since for every $x \in X$ and every $y \in Y$ there is an index $i \in I$ with $x \in X_{i}$ and $y \in Y_{i}$, we obtain $x \perp y$ and hence, $X \subseteq Y^{\perp}$.

Set $\varphi: X \rightarrow Y$ such that $x^{\varphi}=x^{\varphi_{i}}$ for every $x \in X_{i}$ where $i \in I$. By the construction of $\prec$ this map is well-defined. Since for two points $x$ and $x^{\prime}$ of $X$ and a point $y \in Y$ there is a set $X_{i}$ with $i \in I$ such that $\left\{x, x^{\prime}\right\} \leq X_{i}$ and $y \in Y_{i}$, the map $\varphi$ has to be bijective. Hence $(X, Y, \varphi)$ is an upper bound for the chain $\left(X_{i}, Y_{i}, \varphi_{i}\right)_{i \in I}$. We may apply Zorn's Lemma to conclude that there are maximal elements in $\mathcal{M}$ with respect to $\prec$.

Let $(X, Y, \varphi) \in \mathcal{M}$ be such a maximal element. Suppose $X$ and $Y$ are finite. Set $S:=$ $M \cap N$. Then $\operatorname{crk}_{M}(\langle X, S\rangle)$ is infinite since $\operatorname{crk}_{M}(S)$ is infinite. Since $S \leq N \leq Y^{\perp}$, we obtain $\langle X, S\rangle \leq Y^{\perp} \cap M$. Thus, $\left.M \cap Y^{\perp}\right\rangle\langle X, S\rangle$ since $\operatorname{crk}_{M}\left(M \cap Y^{\perp}\right)<\infty$ by Lemma 2.12(i). Let $x \in\left(M \cap Y^{\perp}\right) \backslash\langle X, S\rangle$ and set $X^{\prime}:=X \cup\{x\}$. Then $\left\langle X^{\prime}\right\rangle \cap S=\emptyset$ and therefore $\left\langle X^{\prime}\right\rangle \cap N=\emptyset$. Since $X^{\prime} \subseteq Y^{\perp}$, we obtain $\left.N \cap X^{\prime \perp}\right\rangle\langle Y, S\rangle$ by repeating the same arguments. Let $y \in N \cap X^{\perp^{\perp}} \backslash\langle Y, S\rangle$ and set $Y^{\prime}:=Y \cup\{y\}$. Further let $\varphi^{\prime}: X^{\prime} \rightarrow Y^{\prime}$ be the map with $\left.\varphi^{\prime}\right|_{X}=\varphi$ and $x^{\varphi}=y$. Then $\left(X^{\prime}, Y^{\prime}, \varphi^{\prime}\right) \in \mathcal{M}$ and $(X, Y, \varphi) \prec\left(X^{\prime}, Y^{\prime}, \varphi^{\prime}\right)$, a contradiction. Hence, $X$ and $Y$ have to be infinite sets. Let $L$ be a generator containing $X \cup Y$. Then $\operatorname{crk}_{L}(L \cap M)$ is infinite since $\langle Y\rangle \leq L \backslash M$ and analogously $\operatorname{crk}_{L}(L \cap N)$ is infinite. Thus, $L, M$ and $N$ are contained in three different connected components of the dual polar space of $\mathscr{S}$.

\section{Spanning pairs}

We are now interested in conditions that a polar space has to satisfy in order to be isomorphic to the dual of some ADP. In the following we will give a sufficient condition for this. 
Definition 6.1. Let $M_{0}$ and $M_{1}$ be two generators of a polar space $\mathscr{S}$ such that for every point $p \in \mathscr{S}$ there are points $p_{0} \in M_{0}$ and $p_{1} \in M_{1}$ with $p \in\left(M_{0} \cup\left\{p_{1}\right\}\right)^{\perp \perp} \cap\left(M_{1} \cup\right.$ $\left.\left\{p_{0}\right\}\right)^{\perp \perp}$. Then we call $\left(M_{0}, M_{1}\right)$ a spanning pair.

Proposition 6.2. Let $\left(M_{0}, M_{1}\right)$ be a spanning pair of a polar space $\mathscr{S}$. Then $M_{0} \cap M_{1}=$ $\operatorname{Rad}(\mathscr{S})$.

Proof. Since $M_{0}$ and $M_{1}$ are both maximal, we obtain $\operatorname{Rad}(\mathscr{S}) \leq M_{0} \cap M_{1}$. Now let $p \in \mathscr{S}$ and $q \in M_{0} \cap M_{1}$. Then there is a point $p_{0} \in M_{0}$ such that $p \in\left(M_{1} \cup\left\{p_{0}\right\}\right)^{\perp \perp}$. Thus, $q \perp p$ since $q \in\left(M_{1} \cup\left\{p_{0}\right\}\right)^{\perp}$ and therefore $q \in \operatorname{Rad}(\mathscr{S})$.

A direct consequence of this proposition is that in a non-degenerate polar space the two generators of a spanning pair are always disjoint.

Proposition 6.3. Let $\mathscr{S}$ be a non-degenerate polar space and let $M_{0}$ and $M_{1}$ be two generators of $\mathscr{S}$. Then the following statements are equivalent:

(a) $\left(M_{0}, M_{1}\right)$ is a spanning pair.

(b) For $i \in\{0,1\}$ and $p \in \mathscr{S} \backslash\left(M_{0} \cup M_{1}\right)$, there is a point $p_{i} \in M_{i}$ with $p^{\perp} \cap M_{1-i}=$ $p_{i}{ }^{\perp} \cap M_{1-i}$.

(c) For $i \in\{0,1\}$ and $p \in \mathscr{S} \backslash\left(M_{0} \cup M_{1}\right)$, there is a non-empty subspace $U_{i} \leq M_{i}$ of finite rank with $p^{\perp} \cap M_{1-i} \geq U_{i}^{\perp} \cap M_{1-i}$.

Proof. Note that for (b) and (c) the cases $i=0$ and $i=1$ are analogous.

(a) $\Rightarrow$ (b): Let $p \in \mathscr{S} \backslash M_{1}$. Then there is a point $p_{0} \in M_{0}$ with $p \in\left(M_{1} \cup\left\{p_{0}\right\}\right)^{\perp \perp}$ and hence $\left(M_{1} \cup\left\{p_{0}\right\}\right)^{\perp} \leq p^{\perp}$. Since $M_{1}$ is a generator, we obtain $M_{1}^{\perp}=M_{1}$ and therefore $\left(M_{1} \cup\left\{p_{0}\right\}\right)^{\perp}=p_{0}{ }^{\perp} \cap M_{1}$. The claim follows since $p^{\perp} \cap M_{1}$ is a hyperplane of $M_{1}$.

(b) $\Rightarrow$ (a): Let $p \in \mathscr{S} \backslash\left(M_{0} \cup M_{1}\right)$. Then there is a point $p_{0} \in M_{0}$ such that $p^{\perp} \cap M_{1}=p_{0}^{\perp} \cap M_{1}$. Since $M_{1}$ is a generator, we conclude $\left(M_{1} \cup\left\{p_{0}\right\}\right)^{\perp \perp}=\left(M_{1} \cap\right.$ $\left.p_{0}{ }^{\perp}\right)^{\perp}=\left(M_{1} \cap p^{\perp}\right)^{\perp} \geq\left(p^{\perp}\right)^{\perp} \ni p$. Let $p \in M_{1}$. Then $p \in\left(M_{1} \cup\left\{p_{0}\right\}\right)^{\perp \perp}$ for every choice of $p_{0} \in M_{0}$ since $\left(M_{1} \cup\left\{p_{0}\right\}\right)^{\perp} \leq M_{1}$. Finally, for $p \in M_{0}$ we set $p_{0}:=p$.

(b) $\Rightarrow$ (c): This follows with $U_{i}:=\left\{p_{i}\right\}$.

(c) $\Rightarrow$ (b): Let $p \in \mathscr{S} \backslash\left(M_{0} \cup M_{1}\right)$ and let $U_{0} \leq M_{0}$ be a subspace of finite rank such that $p^{\perp} \cap M_{1} \geq U_{0} \perp \cap M_{1}$. Lemma 2.12(i) implies $\operatorname{crk}_{M_{1}}\left(U_{0}^{\perp} \cap M_{1}\right)<\infty$ and therefore $U_{0}^{\perp} \cap M_{1}$ has a finite corank in $p^{\perp} \cap M_{1}$ that we denote by $k$. If $k>0$ there is a point $q \in\left(p^{\perp} \cap M_{1}\right) \backslash U_{0}{ }^{\perp}$. Set $V_{0}:=q^{\perp} \cap U_{0}$. Since $V_{0}$ is a hyperplane of $U_{0}$, there is a point $u \in U_{0}$ such that $V_{0}{ }^{\perp} \cap u^{\perp}=U_{0}{ }^{\perp}$. Hence, $U_{0}{ }^{\perp}$ is a hyperplane of $V_{0}{ }^{\perp}$ and therefore $V_{0}{ }^{\perp} \cap M_{1}=\left\langle q, U_{0}{ }^{\perp} \cap M_{1}\right\rangle$. Since $q \in p^{\perp}$, we conclude that $V_{0}{ }^{\perp} \cap M_{1}$ has corank $k-1$ in $p^{\perp} \cap M_{1}$. After finitely many steps we end up with a non-empty subspace $V_{0}$ with $p^{\perp} \cap M_{1}=V_{0}^{\perp} \cap M_{1}$. Then $p_{0}^{\perp} \cap M_{1}=p^{\perp} \cap M_{1}$ for any $p_{0} \in V_{0} \backslash M_{1}$.

Our next goal is to show that a non-degenerate polar space with a spanning pair has, in fact, many spanning pairs. More precisely, the spanning pairs form a symmetric, total relation on the set of generators that are commensurate to any generator contained in a spanning pair. Later on, this relation will play the role of an opposition relation in a certain part of the dual polar space. 
Lemma 6.4. Let $\left(M_{0}, M_{1}\right)$ be a spanning pair of a non-degenerate polar space $\mathscr{S}$. Further let $M_{2}$ be a generator with $M_{1} \cap M_{2}=\emptyset$ and $\mathrm{d}^{*}\left(M_{0}, M_{2}\right)=1$. Then $\left(M_{1}, M_{2}\right)$ is a spanning pair.

Proof. Let $p \in \mathscr{S} \backslash\left(M_{1} \cup M_{2}\right)$. We show that there are points $p_{1} \in M_{1}$ and $p_{2} \in M_{2}$ with $p_{1}{ }^{\perp} \cap M_{2}=p^{\perp} \cap M_{2}$ and $p_{2}{ }^{\perp} \cap M_{1}=p^{\perp} \cap M_{1}$.

Let $q \in M_{2} \backslash M_{0}$. Since $p$ and $q$ are not contained in $M_{1}$, there are points $p_{0}$ and $q_{0}$ in $M_{0}$ with $p_{0}^{\perp} \cap M_{1}=p^{\perp} \cap M_{1}=: H_{p}$ and $q_{0}^{\perp} \cap M_{1}=q^{\perp} \cap M_{1}=: H_{q}$. If $H_{p}=H_{q}$ we set $p_{2}:=q$. Otherwise $p_{0} \neq q_{0}$ and the line $p_{0} q_{0}$ meets $M_{2}$ in a point $s$ since $M_{2}$ intersects $M_{0}$ in a hyperplane. We set $H:=H_{p} \cap H_{q}$. Since $H \leq p_{0}{ }^{\perp}$ and $H \leq q_{0}{ }^{\perp}$, we conclude $H \leq s^{\perp}$. Since $H \leq q^{\perp}$, every point on $s q$ is collinear to all points in $H$. Since $H_{q}$ is a hyperplane in $M_{1}, H$ is a hyperplane of $H_{p}$. Let $r \in H_{p} \backslash H$ and let $p_{2} \in s q \cap r^{\perp}$. Then $p_{2}{ }^{\perp}$ contains $\langle r, H\rangle=H_{p}$. Since $p_{2}{ }^{\perp} \cap M_{1}$ is a hyperplane of $M_{1}$, we obtain $p_{2}^{\perp} \cap M_{1}=H_{p}$.

Since $q \notin M_{0} \cup M_{1}$, there is a point $q_{1} \in M_{1}$ with $q_{1}^{\perp} \cap M_{0}=q^{\perp} \cap M_{0}=M_{0} \cap M_{2}$. We may assume $p^{\perp} \cap M_{2} \neq M_{0} \cap M_{2}$ since otherwise we are done by setting $p_{1}:=q_{1}$. Hence, $p \notin M_{0}$ and there is point $r \in M_{1}$ with $r^{\perp} \cap M_{0}=p^{\perp} \cap M_{0}$ and $r \neq q_{1}$. All points on $r q_{1}$ are collinear to all points of $p^{\perp} \cap M_{0} \cap M_{2}$. Since $M_{0} \cap M_{2}$ is a hyperplane of $M_{2}$, the subspace $p^{\perp} \cap M_{0} \cap M_{2}$ is a hyperplane of $p^{\perp} \cap M_{2}$. Let $s \in p^{\perp} \cap M_{2} \backslash M_{0}$ and let $p_{1} \in r q_{1} \cap s^{\perp}$. Then $p_{1}{ }^{\perp}$ contains $\left\langle p^{\perp} \cap M_{0} \cap M_{2}, s\right\rangle=p^{\perp} \cap M_{2}$. Since $p_{1}{ }^{\perp} \cap M_{2}$ is a hyperplane of $M_{2}$, we obtain $p_{1}^{\perp} \cap M_{2}=p^{\perp} \cap M_{2}$.

Lemma 6.5. Let $\left(M_{0}, M_{1}\right)$ be a spanning pair of a non-degenerate polar space $\mathscr{S}$. Let $p_{0} \in M_{0}$ and $p_{1} \in M_{1}$ be two points which are not collinear. Then $\left(p_{1} \oplus M_{0}, p_{0} \oplus M_{1}\right)$ is a spanning pair.

Proof. Set $M_{0}^{\prime}:=p_{1} \oplus M_{0}$ and $M_{1}^{\prime}:=p_{0} \oplus M_{1}$. Since $p_{1} \not \perp p_{0}$, we obtain $p_{1} \notin M_{1}^{\prime}$. Since $M_{1} \leq p_{1}{ }^{\perp}$, the hyperplanes $p_{1} \perp \cap M_{1}^{\prime}$ and $M_{1} \cap M_{1}^{\prime}$ of $M_{1}^{\prime}$ have to be equal. With $p_{1} \in M_{0}^{\prime}$ we conclude $M_{0}^{\prime} \cap M_{1}^{\prime} \leq p_{1} \perp \cap M_{1}^{\prime} \leq M_{1}$. Hence, $M_{0}^{\prime} \cap M_{1}=\left\{p_{1}\right\}$ implies $M_{0}^{\prime} \cap M_{1}^{\prime}=\emptyset$. Let $p \in \mathscr{S} \backslash\left(M_{0}^{\prime} \cup M_{1}^{\prime}\right)$. Because of symmetric reasons, we only show that there is a point $q \in M_{1}^{\prime}$ with $p^{\perp} \cap M_{0}^{\prime}=q^{\perp} \cap M_{0}^{\prime}$. It suffices to show $p^{\perp} \cap M_{0}^{\prime} \leq q^{\perp} \cap M_{0}^{\prime}$ since $q \notin M_{0}^{\prime}$ and hence, $q^{\perp} \cap M_{0}^{\prime}$ and $p^{\perp} \cap M_{0}^{\prime}$ are both hyperplanes in $M_{0}^{\prime}$.

Assume $p \in M_{0}$. Then $p^{\perp} \cap M_{0}^{\prime}=M_{0} \cap M_{0}^{\prime}=p_{0}^{\perp} \cap M_{0}^{\prime}$. Hence $q:=p_{0}$ has the asked property. For $p \in M_{1}$, we obtain $p \neq p_{1}$ since $p_{1} \in M_{0}^{\prime}$. Hence, the line $p p_{1}$ intersects the hyperplane $M_{1} \cap M_{1}^{\prime}$ of $M_{1}$ in a point $q$. Since $p^{\perp} \cap M_{0}^{\prime}$ is contained in $p^{\perp}$ and in $p_{1}^{\perp}$, it is also contained in $q^{\perp}$.

It remains the case $p \notin M_{0} \cup M_{1}$. Let $r \in M_{1}$ be the point with $p^{\perp} \cap M_{0}=r^{\perp} \cap M_{0}$. If $r^{\perp} \cap M_{0}=p_{1} \perp \cap M_{0}$, then $p^{\perp} \cap M_{0}=M_{0} \cap M_{0}^{\prime}$ and therefore $p^{\perp} \cap M_{0}^{\prime}=p_{0}{ }^{\perp} \cap M_{0}^{\prime}$ and we may set $q:=p_{0}$. Otherwise $r \neq p_{1}$ and hence, the line $p_{1} r$ meets $M_{1}^{\prime}$ in a point $q_{1}$ since $M_{1}^{\prime}$ intersects $M_{1}$ in a hyperplane. Furthermore $H:=p^{\perp} \cap M_{0} \cap M_{0}^{\prime}$ is a hyperplane of $p^{\perp} \cap M_{0}^{\prime}$ since $p^{\perp} \cap M_{0}^{\prime} \neq M_{0} \cap M_{0}^{\prime}$. Now $H \leq r^{\perp}$ and $H \leq p_{1} \perp$ implies $H \leq q_{1}{ }^{\perp}$. Let $s \in p^{\perp} \cap M_{0}^{\prime} \backslash H$. Since $q_{1} \in M_{1}^{\prime} \backslash M_{0}$, we obtain $p_{0} \neq q_{1}$ and hence, there is a point $q \in p_{0} q_{1} \cap s^{\perp}$. With $H \leq p_{0}{ }^{\perp}$ and $H \leq q_{1}{ }^{\perp}$ we obtain $H \leq q^{\perp}$ and finally $p^{\perp} \cap M_{0}^{\prime}=\langle s, H\rangle \leq q^{\perp}$. 
Proposition 6.6. Let $\left(M_{0}, M_{1}\right)$ be a spanning pair of a non-degenerate polar space $\mathscr{S}$. Further let $\left(M_{0}^{\prime}, M_{1}^{\prime}\right)$ be a pair of generators with $M_{0}^{\prime} \cap M_{1}^{\prime}=\emptyset, \mathrm{d}^{*}\left(M_{0}, M_{0}^{\prime}\right)=n<\infty$ and $\mathrm{d}^{*}\left(M_{1}, M_{1}^{\prime}\right)=m<\infty$. Then $\left(M_{0}^{\prime}, M_{1}^{\prime}\right)$ is a spanning pair.

Proof. We proceed by induction over $(n, m)$ using the strict total order $\left(n_{0}, m_{0}\right) \prec$ $\left(n_{1}, m_{1}\right)$ if and only if $n_{0}+m_{0}<n_{1}+m_{1}$ or $\left(n_{0}+m_{0}=n_{1}+m_{1} \wedge n_{0}<n_{1}\right)$. If $n+m \leq 1$ the claim follows by Lemma 6.4. So from now on, we assume $n+m \geq 2$.

Assume there is a point $p \in M_{i}^{\prime} \backslash M_{i}$ for $i=0$ or $i=1$ such that $\left(p \oplus M_{i}\right) \cap M_{1-i}=$ $\emptyset$. Then $\left(p \oplus M_{i}, M_{1-i}\right)$ is a spanning pair by Lemma 6.4. Since $\mathrm{d}^{*}\left(p \oplus M_{i}, M_{i}^{\prime}\right)=$ $\mathrm{d}^{*}\left(M_{i}, M_{i}^{\prime}\right)-1$, we may apply the induction hypothesis to prove the claim. Hence, we may now assume that there is no such point.

First assume $n \neq 0$. Let $p \in M_{0}^{\prime} \backslash M_{0}$. Then there is a point $p_{1} \in\left(p \oplus M_{0}\right) \cap M_{1}$. We obtain $p \oplus M_{0}=p_{1} \oplus M_{0}$. Since $M_{0} \cap M_{1}=\emptyset$, there is a point $p_{0} \in M_{0}$ which is not collinear to $p_{1}$. By Lemma 6.5 the pair $\left(p_{1} \oplus M_{0}, p_{0} \oplus M_{1}\right)$ is a spanning pair. Since $\mathrm{d}^{*}\left(p_{1} \oplus M_{0}, M_{0}^{\prime}\right)=n-1$ and $\mathrm{d}^{*}\left(p_{0} \oplus M_{1}, M_{1}^{\prime}\right) \leq m+1$, we may apply the induction hypothesis.

Finally, assume $n=0$ and $m \geq 2$. Then there are generators $N_{i}$ for $0 \leq i \leq m$ and points $s_{i} \in M_{1}^{\prime}$ for $0 \leq i<m$ such that $N_{i+1}=s_{i} \oplus N_{i}, N_{0}=M_{1}$ and $N_{m}=M_{1}^{\prime}$. Let $p_{0} \in N_{1} \cap M_{0}$. Since $M_{0}=M_{0}^{\prime}$ and $M_{0}^{\prime} \cap M_{1}^{\prime}=\emptyset$ there is a point $s_{j}$ for $1 \leq j<m$ not collinear to $p_{0}$. Let $q_{0} \in s_{j} \oplus M_{1} \cap M_{0}$. Since $M_{1}$ intersects $s_{j} \oplus M_{1}$ in a hyperplane, the line $s_{j} q_{0}$ meets $M_{1}$ in a point $q_{1}$. Since $p_{0} \not \perp s_{j}, p_{0} \perp q_{0}$ and $q_{0} \neq q_{1}$, we obtain $p_{0} \not \perp q_{1}$. Now $\left(q_{1} \oplus M_{0}, N_{1}\right)$ is a spanning pair by Lemma 6.5 since $N_{1}=p_{0} \oplus M_{1}$. With $s_{j} \in$ $q_{0} q_{1} \leq q_{1} \oplus M_{0}$ we may use again Lemma 6.5 to conclude that $\left(p_{0} \oplus\left(q_{1} \oplus M_{0}\right), s_{j} \oplus N_{1}\right)$ is a spanning pair. Since $p_{0} \in M_{0} \backslash q_{1} \oplus M_{0}$, we obtain $p_{0} \oplus\left(q_{1} \oplus M_{0}\right)=M_{0}$. With $s_{j} \in M_{1}^{\prime} \backslash N_{1}$ we obtain $\mathrm{d}^{*}\left(s_{j} \oplus N_{1}, M_{1}^{\prime}\right)=\mathrm{d}^{*}\left(N_{1}, M_{1}^{\prime}\right)-1=m-2$. Hence, the claim follows by the induction hypothesis.

Corollary 6.7. Let $\left(M_{0}, M_{1}\right)$ be a spanning pair of a non-degenerate polar space. Further let $\mathcal{G}_{i}$ be the set of all generators that are commensurate with $M_{i}$. Then for every $N_{0} \in \mathcal{G}_{0}$ there is a disjoint generator $N_{1} \in \mathcal{G}_{1}$. Moreover, every pair $\left(N_{0}, N_{1}\right) \in \mathcal{G}_{0} \times \mathcal{G}_{1}$ with $N_{0} \cap N_{1}=\emptyset$ is a spanning pair.

Proof. Let d* $\left(M_{0}, N_{0}\right)=1$. If $N_{0} \cap M_{1}=\emptyset$, we set $N_{1}:=M_{1}$. Otherwise $N_{0} \cap M_{1}$ intersect in a point $p$. Let $q \in M_{0} \backslash p^{\perp}$ and set $N_{1}:=q \oplus M_{1}$. Then $N_{1} \cap N_{0}=\emptyset$ since $\left(N_{0}, N_{1}\right)$ is a spanning pair by Lemma 6.5. Thus, the first claim follows by induction. Applying Proposition 6.6 proves the second claim.

In the following, we denote the dual polar space of a polar space $\mathscr{S}$ by $\mathscr{D}(\mathscr{S})$. Since this is the same notation as we used for ADPs, there might be confusion. Therefore, we will always make clear whether $\mathscr{S}$ is an ADP or a polar space ${ }^{3}$.

Lemma 6.8. Let $X$ and $Y$ be generators of a polar space $\mathscr{S}$ such that $\mathrm{d}^{*}(X, Y)=n<$ $\infty$. Set $G:=\langle X, Y\rangle_{\mathrm{g}} \leq \mathscr{D}(\mathscr{S})$. Then a generator $Z \leq \mathscr{S}$ belongs to $G$ if and only if $Z \geq X \cap Y$.

\footnotetext{
${ }^{3}$ There are point-line spaces, for instance a single line, that are both ADPs and polar spaces. If such a case occurs, it will be clear if this space is treated as an ADP or as a polar space.
} 
Proof. Let $H$ be the set of all generators of $\mathscr{S}$ containing $S:=X \cap Y$. Let $W$ and $Z$ be distinct adjacent generators contained in $H$. Then $W \cap Z \geq S$ and therefore all generators containing $W \cap Z$ belong to $H$. Thus $H$ is a subspace of $\mathscr{D}(\mathscr{S})$. Now let $W$ and $Z$ be two arbitrary generators of $H$ with $\mathrm{d}^{*}(W, Z)=k>1$ and let $V \in \mathscr{D}(\mathscr{S})$ with $\mathrm{d}^{*}(W, V)=k-1$ and $V \sim Z$. Then there is a point $p \in W \cap V \backslash Z$ with $V=p \oplus Z$. Since $W \cap Z \leq p^{\perp}$, we obtain $S \leq W \cap Z \leq V$. Hence, $H$ is convex and therefore $G \leq H$.

To prove $H \leq G$ we proceed by induction over $n$. For $n=0$ there is nothing to prove. For $n=1$ we obtain $H=G$ by the definition of the lines in $\mathscr{D}(\mathscr{S})$. Now let $n>1$ and let $Z$ be a generator of $\mathscr{S}$ with $S \leq Z$. Assume there is a point $p \in Z \cap X \backslash S$. Set $Y^{\prime}:=p \oplus Y$. Then $\mathrm{d}^{*}\left(X, Y^{\prime}\right)=n-1$ and therefore $X \cap Y^{\prime}=\langle p, S\rangle$. Since $\langle p, S\rangle \leq Z$, we may apply the induction hypothesis to conclude $Z \in\left\langle X, Y^{\prime}\right\rangle_{\mathrm{g}}$. Since $Y^{\prime} \in G$, this implies $Z \in G$. Thus we may assume $X \cap Z=S$ and analogously $Y \cap Z=S$.

Let $p \in Z \backslash S$. Set $X^{\prime}:=p \oplus X$ and $Y^{\prime}:=p \oplus Y$. Assume there is a point $q \in Y \backslash S$ with $q \in X^{\prime}$. Then $X^{\prime}=q \oplus X$ and hence $\mathrm{d}^{*}\left(X^{\prime}, Y\right)=n-1$. Thus, $X^{\prime} \in G$ since $X \sim X^{\prime}$. The line $p q$ meets $X$ in a point $r$ since $X$ intersects $X^{\prime}$ in a hyperplane. This implies $r \in X \cap Y^{\prime}$. With $p q \cap Y=\{q\}$ we obtain $r \in X \backslash S$. We conclude $X^{\prime} \cap Y>S$ if and only if $X \cap Y^{\prime}>S$.

First let $X^{\prime} \cap Y=S$. Then $\mathrm{d}^{*}\left(X^{\prime}, Y\right)=n$ and hence $\mathrm{d}^{*}\left(X^{\prime}, Y^{\prime}\right)=n-1$ and $X^{\prime} \cap Y^{\prime}=\langle p, S\rangle$. Since $\langle p, S\rangle \leq Z$ the induction hypothesis provides $Z \in\left\langle X^{\prime}, Y^{\prime}\right\rangle_{\mathrm{g}}$. Since $S \leq X^{\prime} \cap X$ and $\operatorname{crk}_{X}(S)=n$, there is a singular subspace $U \leq X \cap X^{\prime}$ with $\operatorname{rk}(U)=n-2$ and $S \cap U=\emptyset$. Then $S$ is a hyperplane of $U^{\perp} \cap Y$ by Lemma 2.12(i). Hence, there is a point $q \in Y \backslash S$ with $U \leq q^{\perp}$. Set $X^{\prime \prime}:=q \oplus X$. Then $X \cap X^{\prime}=$ $\langle U, S\rangle \leq X^{\prime \prime}$. Thus, $X, X^{\prime}$ and $X^{\prime \prime}$ lie on a common line in $\mathscr{D}(\mathscr{S})$. Since $X \sim X^{\prime \prime}$ and $\mathrm{d}^{*}\left(X^{\prime \prime}, Y\right)=n-1$, we obtain $X^{\prime \prime} \in G$ and therefore $X^{\prime} \in G$. Analogously, $Y^{\prime} \in G$ and therefore $\left\langle X^{\prime}, Y^{\prime}\right\rangle_{\mathrm{g}} \leq G$. This leads to $Z \in G$.

It remains the case $X^{\prime} \cap Y>S$. Hence, we may assume $(r \oplus X) \cap Y>S$ and $(r \oplus Y) \cap X>S$ for every point $r \in Z \backslash S$. Let $q \in X^{\prime} \cap Y \backslash S$. Then $X^{\prime}=q \oplus X$ and therefore $\mathrm{d}^{*}\left(X^{\prime}, Y\right)=n-1$ and $X^{\prime} \in G$. Since $q \in Y \backslash S$, we obtain $q \notin Z$ and since $Z$ is a generator, there is a point $r \in Z \backslash q^{\perp}$. Set $Y^{\prime \prime}:=r \oplus Y$. Then $X^{\prime} \cap Y^{\prime \prime} \leq X^{\prime} \cap Y$ since $X^{\prime} \leq q^{\perp}$ and $q^{\perp} \cap Y^{\prime \prime}=Y \cap Y^{\prime \prime}$. With $S \leq X^{\prime} \cap Y^{\prime \prime}$ and $q \notin Y^{\prime \prime}$ we obtain $X^{\prime} \cap Y^{\prime \prime}=S$ and hence $\mathrm{d}^{*}\left(X^{\prime}, Y^{\prime \prime}\right)=n$. With $p \in Z \cap X^{\prime}$ and $p \notin S$ we obtain $Z \in\left\langle X^{\prime}, Y^{\prime \prime}\right\rangle_{\mathrm{g}}$ as above. Now $r \in Z \backslash S$ implies $(r \oplus Y) \cap X>S$. Hence, $\mathrm{d}^{*}\left(X, Y^{\prime \prime}\right)=n-1$ and therefore $Y^{\prime \prime} \in G$. Thus, $Z \in G$ and we conclude $H=G$.

Now that we know how convex spans of two commensurate generators in a dual polar spaces look like, we are able to check if a dual polar space satisfies the definition of an LDP. Before doing so we consider two special situations. First we show for a spanning pair $\left(M_{0}, M_{1}\right)$ that the convex span of $M_{0}$ and a commensurate generator $X$ contains a unique generator which has maximal possible intersection with $M_{1}$.

Lemma 6.9. Let $\left(M_{0}, M_{1}\right)$ be a spanning pair of a non-degenerate polar space. Further let $X$ be a generator with $\mathrm{d}^{*}\left(M_{0}, X\right)=k<\infty$. Then there is a generator $Y$ with $Y \cap M_{0}=X \cap M_{0}$ and $\operatorname{rk}\left(Y \cap M_{1}\right)=k-1$. This generator is unique and satisfies $Y=\left(X \cap M_{0}\right) \oplus M_{1}=\left(Y \cap M_{1}\right) \oplus M_{0}$. 
Proof. Set $H:=M_{0} \cap X$. For $0 \leq i<k$, we define recursively points $b_{i} \in X$ such that $\left\{b_{j} \mid j \leq i\right\}^{\perp} \cap M_{0}<\left\{b_{j} \mid j \leq i-1\right\}^{\perp} \cap M_{0}$. Then $\operatorname{crk}_{M_{0}}\left(M_{0} \cap\left\{b_{j} \mid j<i\right\}^{\perp}\right)=i$ and hence $M_{0} \cap\left\{b_{j} \mid j<k\right\}^{\perp}=H$. Since $\left(M_{0}, M_{1}\right)$ is a spanning pair and $b_{i} \notin M_{0}$, there is a point $p_{i} \in M_{1}$ with $b_{i}{ }^{\perp} \cap M_{0}=p_{i}{ }^{\perp} \cap M_{0}$ for $i<k$. We obtain $\left\{p_{i} \mid i<\right.$ $k\}^{\perp} \cap M_{0}=H$ and therefore $\operatorname{rk}\left(\left\langle p_{i} \mid i<k\right\rangle\right)=k-1$ by Lemma 2.12(i). We conclude with Lemma 2.12(ii) that $Y:=\left\{p_{i} \mid i \in k\right\} \oplus M_{0}$ is a generator with $\mathrm{d}^{*}\left(M_{0}, Y\right)=k$ and hence $Y \cap M_{0}=H$.

Since $Y=\left\langle H, p_{i} \mid i<k\right\rangle, H \leq M_{0}$ and $\left\langle p_{i} \mid i<k\right\rangle \leq M_{1}$, we obtain $Y \cap M_{1}=$ $\left\langle p_{i} \mid i<k\right\rangle$ and therefore $Y=\left(Y \cap M_{1}\right) \oplus M_{0}$. Now let $p$ be any point of $H^{\perp} \cap M_{1}$. Then $p$ is collinear to $p_{i}$ for $i<k$. Hence, $p^{\perp} \geq\left\langle H, p_{i}\right| i\langle k\rangle=Y$ and $\langle p, Y\rangle$ is again singular. Thus, $p \in Y$ since $Y$ is a generator. Therefore, $Y$ is uniquely determined and $Y=\left(X \cap M_{0}\right) \oplus M_{1}$.

In the following lemma we show for a more general situation that whenever we have a convex span of two commensurate generators, we can choose these two generators such that one has maximal intersection and the other one has minimal intersection to a certain generator.

Lemma 6.10. Let $\left(M_{0}, M_{1}\right)$ be a spanning pair of a polar space. Further let $X, Y$ and $Z$ be generators such that $X$ and $M_{0}$ are commensurate and $Y, Z$ and $M_{1}$ are commensurate. Set $V:=Y \cap Z$. Then there are generators $Y^{\prime}$ and $Z^{\prime}$ with $Y^{\prime} \cap X=$ $V \cap X, \operatorname{crk}_{Z^{\prime} \cap X}(V \cap X)=\mathrm{d}^{*}(Y, Z)$ and $Y^{\prime} \cap Z^{\prime}=V$.

Proof. By Corollary 6.7 there is a generator $M$ with $\mathrm{d}^{*}\left(M_{1}, M\right)<\infty$ such that $(X, M)$ is a spanning pair. Then $\mathrm{d}^{*}(M, Y)$ and $\mathrm{d}^{*}(M, Z)$ are finite. Hence, we may assume $X=M_{0}$ and $M_{1}=M$.

Set $Y_{X}:=(M \cap Y) \oplus X, Z_{X}:=(M \cap Z) \oplus X$ and $U:=\left\langle X \cap Y_{X}, X \cap Z_{X}\right\rangle$. Then $Y_{X}$ and $Z_{X}$ are generators with $\mathrm{d}^{*}\left(M, Y_{X}\right)<\infty$ and $\mathrm{d}^{*}\left(M, Z_{X}\right)<\infty$ by Lemma 6.9. Since $X \cap M=\emptyset$, this implies that $X \cap Y_{X}$ and $X \cap Z_{X}$ have both finite rank and therefore $\operatorname{rk}(U)<\infty$. By Lemma 6.9 we obtain $Y_{X} \cap M=Y \cap M$ and $\left(X \cap Y_{X}\right)^{\perp} \cap M=Y_{X} \cap M$ and the corresponding for $Z_{X}$. Hence,

$$
\begin{aligned}
U^{\perp} \cap M & =\left(\left(X \cap Y_{X}\right)^{\perp} \cap\left(X \cap Z_{X}\right)^{\perp}\right) \cap M \\
& \left.=\left(\left(X \cap Y_{X}\right)^{\perp} \cap M\right) \cap\left(\left(X \cap Z_{X}\right)^{\perp}\right) \cap M\right) \\
& =\left(Y_{X} \cap M\right) \cap\left(Z_{X} \cap M\right)=(Y \cap M) \cap(Z \cap M)=V \cap M .
\end{aligned}
$$

Thus, $V_{X}:=\langle U, V \cap M\rangle$ is a generator by Lemma 2.12(ii). Now let $B$ be a basis of $V$ containing a basis $B_{0}$ of $V \cap M$ and a basis $B_{1}$ of $V \cap V_{X}$. This is possible since $V \cap M \leq V_{X}$ and hence $B_{0} \subseteq B_{1}$. Since $V_{X}=\langle U, V \cap M\rangle$, every subspace of $V_{X}$ that contains $V \cap M$ has a basis contained in $M \cup X$. Hence, we may assume that we chose $B$ such that $B_{1} \backslash B_{0} \subseteq X$. Since $V_{X}$ is a generator, we obtain $(V \cap M)^{\perp} \cap X=U$. Hence, $V^{\perp} \cap X \leq U$ and therefore $V \cap X \leq U$. With $X \cap M=\emptyset$ we conclude $\left\langle B_{1} \backslash B_{0}\right\rangle=V \cap X$.

Set $B_{V}:=B \backslash B_{1}$ and set $Z^{\prime}:=B_{V} \oplus V_{X}$. Then $\left\langle B_{V}\right\rangle$ is disjoint to $V_{X}$ since $B_{1}$ is a basis of $V \cap V_{X}$. Since $\operatorname{crk}_{V_{X}}\left(V \cap V_{X}\right)$ is finite, $Z^{\prime}$ is a generator with $\mathrm{d}^{*}\left(V_{X}, Z^{\prime}\right)=\left|B_{V}\right|$ 
by Lemma 2.12(ii). Since $B_{1} \subseteq B_{V}{ }^{\perp} \cap V_{X}$, we obtain $B \subseteq Z^{\prime}$ and hence $V \leq Z^{\prime}$. Therefore, $Z^{\prime} \cap X \leq(V \cap M)^{\perp} \cap X=U$. Since $Z^{\prime} \geq V \cap M$ and $V_{X}=\langle U, V \cap M\rangle$, this leads to $\operatorname{crk}_{V_{X} \cap X}\left(Z^{\prime} \cap X\right)=\mathrm{d}^{*}\left(V_{X}, Z^{\prime}\right)$. Hence,

$$
\begin{aligned}
\operatorname{crk}_{Z^{\prime} \cap X}(V \cap X) & =\operatorname{crk}_{V_{X} \cap X}(V \cap X)-\operatorname{crk}_{V_{X} \cap X}\left(Z^{\prime} \cap X\right) \\
& =\operatorname{crk}_{\left\langle V_{X} \cap X, B_{0}\right\rangle}\left(\left\langle V \cap X, B_{0}\right\rangle\right)-\mathrm{d}^{*}\left(V_{X}, Z^{\prime}\right) \\
& =\operatorname{crk}_{V_{X}}\left(\left\langle B_{1}\right\rangle\right)-\left|B_{V}\right|=\operatorname{crk}_{Y}\left(\left\langle B_{1}\right\rangle\right)-\left|B_{V}\right| \\
& =\operatorname{crk}_{Y}\left(\left\langle B_{1}, B_{V}\right\rangle\right)=\mathrm{d}^{*}(Y, Z) .
\end{aligned}
$$

Set $Y_{0}:=Y$. Let $i<\mathrm{d}^{*}(Y, Z)$ such that $Y_{i}$ exists and $Y_{i} \cap X \not \leq Z$. Then let $y_{i} \in Y_{i} \cap X \backslash$ $Z$. Since $Z$ is a generator, there is a point $z_{i} \in Z$ not collinear to $y_{i}$. Set $Y_{i+1}:=z_{i} \oplus Y_{i}$. Since $Y_{i+1} \cap X \leq y_{i}{ }^{\perp}$ and $y_{i}{ }^{\perp} \cap Y_{i+1} \leq Y_{i}$, we conclude $Y_{i} \cap X \geq Y_{i+1} \cap X$. Together with $y_{i} \in\left(Y_{i} \cap X\right) \backslash Y_{i+1}$ we obtain $Y_{i} \cap X>Y_{i+1} \cap X$. After finitely many steps we get a generator $Y_{j}$ for some $j \leq \mathrm{d}^{*}(Y, Z)$ with $Y_{j} \cap X \leq Z$. Set $Y^{\prime}:=Y_{j}$. Then $Y^{\prime} \cap X \leq V \cap X$ since $Y^{\prime} \cap X \leq Y$. On the other hand $V \leq Y_{0}$ and $V \leq z_{i}{ }^{\perp}$ for every $i \leq j$ leads to $V \leq Y^{\prime}$ and thus $Y^{\prime} \cap X=V \cap X$. Now $V \leq Y^{\prime} \cap Z^{\prime}$ implies $\mathrm{d}^{*}\left(Y^{\prime}, Z^{\prime}\right) \leq \mathrm{d}^{*}(Y, Z)$. Hence, with $\operatorname{crk}_{Z^{\prime} \cap X}(V \cap X)=\mathrm{d}^{*}(Y, Z)$ we obtain equality in both inequations.

The following proposition is actually the condition given in Definition 3.4 in terms of polar spaces.

Proposition 6.11. Let $\left(M_{0}, M_{1}\right)$ be a spanning pair of a non-degenerate polar space. Further let $X, Y$ and $Z$ be generators such that $X$ and $M_{0}$ are commensurate and $Y, Z$ and $M_{1}$ are commensurate. Then there is a generator $W$ with $\mathrm{d}^{*}(W, X)=\mathrm{d}^{*}(Y, Z)=: n$ such that for each generator $U \geq W \cap X$ there is exactly one generator $V \geq Y \cap Z$ with $\operatorname{crk}_{U \cap V}(U \cap Y \cap Z)=n$ and $U \cap Y \cap Z=X \cap Y \cap Z$.

Proof. Set $R:=X \cap Y \cap Z$ and $m:=\operatorname{rk}(R)+1$. Since $M_{0} \cap M_{1}=\emptyset$, we obtain $\operatorname{rk}\left(X \cap M_{1}\right)<\infty$ and consequently $\operatorname{rk}(X \cap Y)<\infty$. Thus, $m$ is finite. By Lemma 6.10 we may assume that $X \cap Y=R$ and $\operatorname{crk}_{X \cap Z}(R)=n$. Hence, $\operatorname{rk}(X \cap Z)=n+m-1$.

Let $B$ be a basis of $Y$ containing a basis $B_{0}$ of $Y \cap Z$. Set $B_{1}:=B \backslash B_{0}$. Then $\left\langle B_{1}\right\rangle \cap X \leq Y \cap X=R$. Since $R \leq Y \cap Z$ and $\left\langle B_{1}\right\rangle \subseteq Y \backslash Z$, this implies $\left\langle B_{1}\right\rangle \cap X=\emptyset$. Thus, $W:=B_{1} \oplus X$ is a generator with $\mathrm{d}^{*}(W, X)=n$ by Lemma 2.12(ii). With $B_{1} \subseteq W$ and $B_{1}{ }^{\perp} \cap Z \leq Y$ we obtain $W \cap Z \leq W \cap Y$. Further $W=\left\langle B_{1}, B_{1}{ }^{\perp} \cap X\right\rangle$ yields $W \cap Y \leq\left\langle B_{1}, Y \cap X\right\rangle$. Hence, $W \cap Z \leq\left\langle B_{1}, R\right\rangle$ and since $R \leq Z$ and $\left\langle B_{1}\right\rangle \cap Z=\emptyset$, we conclude $W \cap Z \leq R$. Finally, $R \leq B_{1}{ }^{\perp}$ implies $W \cap Z=R$.

Set $S:=(W \cap X)^{\perp} \cap Z$. Let $C$ be a set of $n$ points such that $\langle C, W \cap X\rangle=W$. Then $C^{\perp} \cap S=C^{\perp} \cap(W \cap X)^{\perp} \cap Z=\langle C, W \cap X\rangle^{\perp} \cap Z=W \cap Z=R$. For every point $p$ the subspace $Z \cap p^{\perp}$ is a hyperplane of $Z$ or equals $Z$. Thus, $\operatorname{crk}_{Z}\left(C^{\perp} \cap Z\right) \leq n$ and $\operatorname{crk}_{S}(R) \leq n$. With $X \leq(W \cap X)^{\perp}$ we obtain $X \cap Z \leq S$. Now $\operatorname{crk}_{X \cap Z}(R)=n$ implies $S=X \cap Z$. Hence, $Y \cap S \leq Y \cap X=R$ and therefore $Y \cap Z \cap(W \cap X)^{\perp}=R$.

Let $U$ be a generator containing $W \cap X$. Then $U \cap Y \cap Z=R$, since $U \geq R$ and $(W \cap X)^{\perp} \cap Y \cap Z=R$. Applying Lemma 6.10 there is a generator $V \geq Y \cap Z$ with $\operatorname{crk}_{V \cap U}(Y \cap Z \cap U)=n$. Hence, there is a subspace $V^{\prime} \subseteq(U \cap V) \backslash(Y \cap Z)$ of rank 
$n-1$. Since $\operatorname{crk}_{V}(Y \cap Z)=\operatorname{crk}_{Y}(Y \cap Z)=n$ this implies $V=\left\langle V^{\prime}, Y \cap Z\right\rangle$. Thus, $V^{\prime} \leq(Y \cap Z)^{\perp}$ and therefore $V=(Y \cap Z) \oplus U$. This proves the uniqueness of $V$.

Theorem 6.12. Let $\mathscr{S}$ be a non-degenerate polar space with a spanning pair $(M, N)$. Then ${ }_{M} \mathscr{D}(\mathscr{S}) \cup{ }_{N} \mathscr{D}(\mathscr{S})$ is an $A D P$.

Proof. Set $\mathscr{W}:={ }_{M} \mathscr{D}(\mathscr{S}) \cup{ }_{N} \mathscr{D}(\mathscr{S})$. For two generators $X$ and $Y$ of $\mathscr{S}$, which are contained in $\mathscr{W}$, we will write $X \leftrightarrow Y$ if and only if $(X, Y)$ is spanning pair. By Corollary 6.7 there is for every generator $X \in{ }_{M} \mathscr{D}(\mathscr{S})$ a generator $Y \in{ }_{N} \mathscr{D}(\mathscr{S})$ with $X \leftrightarrow Y$ and vice versa. Hence, the relation $\leftrightarrow$ is a symmetric, total relation on the points of $\mathscr{W}$. We show that $\mathscr{W}$ is an ADP in which $\leftrightarrow$ is an opposition relation.

Let $X$ and $Y$ be two generators of $\mathscr{S}$ with $\operatorname{rk}(X \cap Y)=n<\infty$. Assume there is a point $p \in X \cap Y$. Then there is a point $q$ with $q \not p$. Set $Y^{\prime}:=q \oplus Y$. Then $p^{\perp} \cap Y^{\prime} \leq Y$ and hence $X \cap Y^{\prime} \leq X \cap Y$. With $p \notin X \cap Y^{\prime}$ and $Y \sim Y^{\prime}$ we conclude $\operatorname{cod}(X, Y)=n+1$ in $\mathscr{W}$ with Corollary 6.7. Thus, $\operatorname{cod}(X, Y)=\operatorname{rk}(X \cap Y)+1$ by the Propositions 6.2 and 2.10 .

Now let $X, Y$ and $Z$ be generators with $\mathrm{d}^{*}(Y, Z)=n<\infty$ and $\min \{\operatorname{cod}(X, V)$ $\left.V \in\langle Y, Z\rangle_{\mathrm{g}}\right\}=m<\infty$. With Lemma 6.8 this means $\operatorname{rk}(X \cap V) \geq m-1$ for every generator $V \geq Y \cap Z$, where equality holds at least one such generator. Hence, $\operatorname{rk}(X \cap Y \cap Z)=m-1$ by Lemma 6.10. By Proposition 6.11 there is a generator $W$ with $\mathrm{d}^{*}(W, X)=n$ such that for every generator $U \in\langle W, X\rangle_{\mathrm{g}}$ there is a unique generator $V \in\langle Y, Z\rangle_{\mathrm{g}}$ with $\operatorname{crk}_{U \cap V}(X \cap Y \cap Z)=n$. Thus, $\operatorname{cod}(U, V)=m+n$. Since $X \cap Y \cap Z=U \cap Y \cap Z$ by Proposition 6.11 and $\operatorname{crk}_{V^{\prime}}(Y \cap Z)=n$ for every generator $V^{\prime} \geq Y \cap Z$, we obtain $\operatorname{rk}\left(U \cap V^{\prime}\right) \leq n+m-1$ and equivalently $\operatorname{cod}\left(U, V^{\prime}\right) \leq n+m$. We conclude that $\langle W, X\rangle_{\mathrm{g}}$ is coparallel to $\langle Y, Z\rangle_{\mathrm{g}}$ with $\operatorname{cod}\left(\langle W, X\rangle_{\mathrm{g}},\langle Y, Z\rangle_{\mathrm{g}}\right)=m+n$. Thus, $\mathscr{W}$ is an LDP by Definition 3.4.

If ${ }_{M} \mathscr{D}(\mathscr{S})={ }_{N} \mathscr{D}(\mathscr{S})$, then $\operatorname{crk}_{M}(M \cap N)<\infty$ and hence $\operatorname{rk}(M)<\infty$. Thus, $\mathscr{W}=\mathscr{D}(\mathscr{S})$ has finite diameter $\operatorname{rk}(M)+1$. If ${ }_{M} \mathscr{D}(\mathscr{S}) \neq{ }_{N} \mathscr{D}(\mathscr{S})$ are distinct connected components, then $\operatorname{rk}(M)=\infty$. For a subspace $U<M$ with $\operatorname{rk}(U)<\infty$ we obtain $\mathrm{d}^{*}(U \oplus N, N)=\operatorname{rk}(U)+1$. Hence, ${ }_{N} \mathscr{D}(\mathscr{S})$ has infinite diameter and analogously $\operatorname{diam}(M \mathscr{D}(\mathscr{S}))=\infty$. Since $(M, N)$ is a spanning pair, $\mathscr{W}$ is irreducible. In both cases $\mathscr{W}$ is an ADP.

Note that in this proof we showed $\operatorname{cod}(M, N)=\operatorname{rk}(M \cap N)+1$ for two generators $M$ and $N$. With Lemma 2.10 this implies that the spanning pairs generate the standard opposition relation if the rank of $\mathscr{S}$ is finite. If $\mathscr{S}$ has infinite rank, then this implies that the opposition relation we get is a reduced one.

\section{From polar spaces to local dual polar spaces and back}

Let $\beta=(M, N)$ be a spanning pair of a polar space $\mathscr{S}$. Then we call $\mathscr{D}_{\beta}(\mathscr{S}):=$ ${ }_{M} \mathscr{D}(\mathscr{S}) \cup{ }_{N} \mathscr{D}(\mathscr{S})$ the atomic dual polar space of $\mathscr{S}$ with respect to $\beta$. We call a space an atomic dual polar space if it is isomorphic to an atomic dual polar space of a polar space. 
Lemma 7.1. Let $\beta$ be a spanning pair of a polar space $\mathscr{S}$. Further let $X$ and $Y$ be two generators with $\operatorname{cod}(X, Y)<\infty$ in $\mathscr{D}_{\beta}(\mathscr{S})$. Then $Z \in\langle X, Y\rangle_{\mathrm{g}} \Leftrightarrow Z \geq X \cap Y$ for every generator $Z \in \mathscr{D}_{\beta}(\mathscr{S})$.

Proof. Set $\mathscr{W}:=\mathscr{D}_{\beta}(\mathscr{S})$. If $\operatorname{diam}(\mathscr{W})<\infty$ this is a consequence of Lemma 6.8 . Hence, we may assume $\mathrm{d}^{*}(X, Y)=\infty$.

Let $\mathrm{d}^{*}(Z, Y)+\operatorname{cod}(X, Y)=\operatorname{cod}(X, Z)$ or equivalently $\operatorname{crk}_{Z}(Y \cap Z)+\operatorname{rk}(X \cap Y)=$ $\operatorname{rk}(X \cap Z)$. With $\operatorname{rk}(X \cap Z)-\operatorname{rk}(X \cap Y) \leq \operatorname{crk}_{X \cap Z}(X \cap Y \cap Z) \leq \operatorname{crk}_{Z}(Y \cap Z)$ this implies $X \cap Y=X \cap Y \cap Z$ and hence, $X \cap Y \leq Z$. Now Lemma 6.8 implies $V \geq X \cap Y$ for every $V \in\langle Y, Z\rangle_{\mathrm{g}}$. Thus, $V \geq X \cap Y$ for every $V \in\langle X, Y\rangle_{\mathrm{g}}$ by Lemma 5.8.

Now let $Z \in{ }_{Y} \mathscr{W}$ be a generator with $Z \geq X \cap Y$. Then by Lemma 6.10 there is a generator $V$ with $V \geq Y \cap Z$ and $\mathrm{d}^{*}(Y, Z)=\operatorname{crk}_{X \cap V}(X \cap Y \cap Z)=\operatorname{crk}_{X \cap V}(X \cap Y)$. Hence, $\operatorname{crk}_{V}(V \cap Y) \geq \mathrm{d}^{*}(Y, Z)$. With $\operatorname{crk}_{Y}(V \cap Y) \leq \operatorname{crk}_{Y}(Y \cap Z)=\mathrm{d}^{*}(Y, Z)$ this implies $\mathrm{d}^{*}(Y, V)=\mathrm{d}^{*}(Y, Z)$ and $V \cap Y=Z \cap V$. Thus, $\mathrm{d}^{*}(V, Y)=\operatorname{rk}(X \cap$ $V)-\operatorname{rk}(X \cap Y)$ and therefore $V \in\left\langle\langle X, Y\rangle_{\mathrm{g}}\right.$. The claim follows since $Z \in\langle V, Y\rangle_{\mathrm{g}}$ by Lemma 6.8.

If $\mathrm{d}(X, Y)=\infty$ there are generators $V \geq X \cap Y$ with $V \notin\left\langle\langle X, Y\rangle_{\mathrm{g}}\right.$. In this case we obtain $\mathrm{d}^{*}(X, V)=\infty$ and $\mathrm{d}^{*}(Y, V)=\infty$. This is a consequence of Proposition 5.22.

Proposition 7.2. Let $\mathscr{S}$ be a non-degenerate polar space with a spanning pair $\left(M_{0}, M_{1}\right)$. Then $\mathscr{D}\left(\mathscr{D}_{\left(M_{0}, M_{1}\right)}(\mathscr{S})\right) \cong \mathscr{S}$.

Proof. Set $\mathscr{W}:=\mathscr{D}_{\left(M_{0}, M_{1}\right)}(\mathscr{S})$ and let $P \in \mathcal{U}^{1}(\mathscr{W})$. Then there are two generators $X$ and $Y$ in $\mathscr{W}$ with $P=\left\langle\langle X, Y\rangle_{\mathrm{g}}\right.$ and $X \cap Y=\{p\}$ for some $p \in \mathscr{S}$. We define $\varphi: \mathcal{U}^{1}(\mathscr{W}) \rightarrow \mathscr{S}$ to be the unique map with $\bigcap_{M \in P} M=\left\{P^{\varphi}\right\}$ for every $P \in \mathcal{U}^{1}(\mathscr{W})$.

Let $p \in \mathscr{S}$. If $p \in M_{0}$, then $M_{0} \cap\left(p \oplus M_{1}\right)=\{p\}$ and hence $P:=\left\langle M_{0}, p \oplus M_{1}\right\rangle_{\mathrm{g}} \in$ $\mathcal{U}^{1}(\mathscr{W})$ with $P^{\varphi}=p$. Analogously, $p=\left\langle\left\langle M_{1}, p \oplus M_{0}\right\rangle_{\mathrm{g}}^{\varphi}\right.$ if $p \in M_{1}$. Now assume $p \notin M_{0} \cup M_{1}$. Set $M_{0}^{\prime}:=p \oplus M_{0}$. If $M_{0}^{\prime} \cap M_{1}=\emptyset$, then $\left\langle\left\langle M_{0}^{\prime}, p \oplus M_{1}\right\rangle\right\rangle_{\mathrm{g}}^{\varphi}=p$ as above. If $M_{0}^{\prime} \cap M_{1} \neq \emptyset$, then $q \oplus M_{0}=M_{0}^{\prime}$ for some $q \in M_{0}^{\prime} \cap M_{1}$. Let $r \in M_{0} \backslash q^{\perp}$ and set $M_{1}^{\prime}:=r \oplus M_{1}$ then $\left(M_{0}^{\prime}, M_{1}^{\prime}\right)$ is a spanning pair by Lemma 6.5 and therefore $\left\langle M_{0}^{\prime}, p \oplus M_{1}^{\prime}\right\rangle_{\mathrm{g}}^{\varphi}=p$. Hence, $\varphi$ is surjective. By Lemma 7.1 the coconvex span of two generators is uniquely determined by their intersection. Thus, $\varphi$ is bijective.

Now let $X$ and $Y$ be two generators with $\operatorname{cod}(X, Y)=2$. Then $l:=X \cap Y$ is a line of $\mathscr{S}$. Set $L:=\langle X, Y\rangle_{\mathrm{g}}$ and $[L]:=\left\{P \in \mathcal{U}^{1}(\mathscr{W}) \mid P \geq L\right\}$. Let $P \in \mathcal{U}^{1}(\mathscr{W})$. If $P \in[L]$, then $P^{\varphi} \in X \cap Y=l$. On the other hand if $Q^{\varphi} \in l$, then $X$ and $Y$ are elements of $Q$ and therefore $L \leq Q$. We conclude $[L]^{\varphi}=l$. Thus, $\varphi$ is an isomorphism from $\mathscr{D}(\mathscr{W})$ to $\mathscr{S}$.

Proposition 7.3. Every ADP is an atomic dual polar space. Moreover, let $\mathscr{S}$ be an ADP with $\operatorname{diam}(\mathscr{S}) \geq 1$ and let $p$ and $q$ be opposite points. Set $P:=\left\{U \in \mathcal{U}^{1}(\mathscr{S}) \mid p \in U\right\}$ and $Q:=\left\{U \in \mathcal{U}^{1}(\mathscr{S}) \mid q \in U\right\}$. Then $(P, Q)$ is a spanning pair of $\mathscr{D}(\mathscr{S})$ and $\mathscr{D}_{(P, Q)}(\mathscr{D}(\mathscr{S})) \cong \mathscr{S}$. 
Proof. By Theorem 5.19 $\mathscr{D}(\mathscr{S})$ is non-degenerate polar space and by Lemma $5.20 P$ and $Q$ are generators. Now let $M \in \mathcal{U}^{1}(\mathscr{S})$ with $M \notin P \cup Q$. Then $\mathrm{d}(p, M)=1$ by Lemma 5.13. Set $p^{\prime}:=\operatorname{pr}_{M}(p)$ and $q^{\prime}:=\operatorname{copr}_{p p^{\prime}}(q)$. Then $\operatorname{cod}\left(q, q^{\prime}\right)=1$ since $p \leftrightarrow q$ and therefore $N:=\left\langle q q, q^{\prime}\right\rangle_{\mathrm{g}} \in Q$. If every generator of $\mathscr{D}(\mathscr{S})$ is a singleton, then $M^{\perp} \cap P=N^{\perp} \cap P=\emptyset$. Now assume that every generator contains a line. Then for every $K \in P$ with $K \sim M$ there is a point $x \in K \cap M$. Since $p^{\prime}$ is the gate for $p$ in $M$, we obtain $p^{\prime} \in\langle p p, x\rangle_{\mathrm{g}} \leq K$. With $q^{\prime} \in p p^{\prime}$ this implies $q^{\prime} \in K$ and therefore $K \sim N$. Conversely, let $K \in P$ with $K \sim N$. Then there is a point $y \in K \cap N$. Since $p \leftrightarrow q$ and $\mathrm{d}\left(p, q^{\prime}\right)=1$, we know $p \notin N$ and hence, $q^{\prime}$ is the gate for $p$ in $N$. This implies $q^{\prime} \in\langle p, y\rangle_{\mathrm{g}} \leq K$. Thus, $p^{\prime} \in p q^{\prime} \leq K$ and therefore $K \sim M$. We conclude $M^{\perp} \cap P=N^{\perp} \cap P$ and hence, $(P, Q)$ is a spanning pair.

Let $\mathcal{G}$ be the set of generators of $\mathscr{D}(\mathscr{S})$ and $\operatorname{set} \varphi: \mathscr{S} \rightarrow \mathcal{G}: p \mapsto\left\{M \in \mathcal{U}^{1}(\mathscr{S}) \mid p \in\right.$ $M\}$. For the case $\operatorname{diam}(\mathscr{S})<\infty$, we have seen in the proof of Theorem 4.9 that $\varphi$ is an isomorphism from $\mathscr{S}$ to the dual polar space of $\mathscr{D}(\mathscr{S})$. Hence, $\mathscr{D}(\mathscr{D}(\mathscr{S}))$ is connected and therefore equal to $\mathscr{D}_{(P, Q)}(\mathscr{D}(\mathscr{S}))$. In the case $\operatorname{diam}(\mathscr{S})=\infty$ we have seen in the proof of Theorem 5.21 that $\mathscr{S}$ and $\mathscr{S}^{\varphi}$ are isomorphic and that $\mathscr{S}^{\varphi}$ consists of exactly two connected components of $\mathscr{D}(\mathscr{D}(\mathscr{S}))$. With $\{P, Q\} \subseteq \mathscr{S}^{\varphi}$ and $\mathrm{d}^{*}(P, Q)=\infty$ we conclude $\mathscr{S}^{\varphi}=\mathscr{D}_{(P, Q)}(\mathscr{D}(\mathscr{S}))$.

It remains the case where $\mathscr{S}$ is a singleton. Then $\beta:=(\mathscr{S}, \mathscr{S})$ is a spanning pair of the polar space $\mathscr{S}$ and the atomic dual polar space $\mathscr{D}_{\beta}(\mathscr{S})$ is a singleton.

By Theorem 6.12 every atomic dual polar space is an ADP. Hence, from now on we may cancel the "local" in "atomic local dual polar space" and use the abbreviation ADP also for atomic dual polar spaces. We call two ADPs isomorphic if there is an isomorphism between the underlying point-line spaces that preserves the opposition relation.

Definition 7.4. Two ADPs are dual-isomorphic if their duals are isomorphic. For two polar spaces $\mathscr{S}$ and $\mathscr{S}^{\prime}$ with a spanning pair, we use the term dual-isomorphic if there is an atomic dual polar space of $\mathscr{S}$ that is isomorphic to an atomic dual polar space of $\mathscr{S}^{\prime}$.

Theorem 7.5. There is a one-to-one correspondence between the equivalence classes of dual-isomorphic polar spaces with a spanning pair and the equivalence classes of dualisomorphic ADPs.

Proof. By Theorem 6.12 every polar space with a spanning pair gives rise to an ADP and by Theorem 5.19 every ADP gives rise to a polar space with a spanning pair. Thus, it remains to show for an ADP $\mathscr{W}$ and a polar space $\mathscr{S}$ with a spanning pair $\alpha$ that $\mathscr{D}(\mathscr{W})$ and $\mathscr{S}$ are dual-isomorphic if and only if $\mathscr{W}$ and $\mathscr{D}_{\alpha}(\mathscr{S})$ are dual-isomorphic.

Let $\mathscr{W}$ be an ADP and let $\mathscr{S}$ be a polar space with a spanning pair. Assume $\mathscr{D}(\mathscr{W})$ and $\mathscr{S}$ are dual-isomorphic. Let $\alpha$ be a spanning pair of $\mathscr{S}$ and let $\beta$ be a spanning pair of $\mathscr{D}(\mathscr{W})$ such that $\mathscr{D}_{\alpha}(\mathscr{S}) \cong \mathscr{D}_{\beta}(\mathscr{D}(\mathscr{W}))$. If $\mathscr{W}$ contains a line, then $\mathscr{D}(\mathscr{W})$ is a nondegenerate polar space by Theorem 5.19. Hence, we obtain $\mathscr{D}(\mathscr{W}) \cong \mathscr{D}\left(\mathscr{D}_{\beta}(\mathscr{D}(\mathscr{W}))\right) \cong$ $\mathscr{D}\left(\mathscr{D}_{\alpha}(\mathscr{S})\right)$ by Proposition 7.2 and therefore $\mathscr{W}$ and $\mathscr{D}_{\alpha}(\mathscr{S})$ are dual-isomorphic. If $\mathscr{W}$ is a singleton then $\mathscr{D}(\mathscr{W})=\{\emptyset\}$ and $\mathscr{D}(\mathscr{D}(\mathscr{W}))$ is again a singleton. This implies that 
$\mathscr{S}$ has to be singular and $\mathscr{D}(\mathscr{S})=\{\mathscr{S}\}$ is the only ADP of $\mathscr{S}$. Hence, $\mathscr{D}(\mathscr{S}) \cong \mathscr{W}$ and therefore $\mathscr{W}$ and $\mathscr{D}(\mathscr{S})$ are dual-isomorphic.

Now assume that $\mathscr{S}$ possesses a spanning pair $\alpha$ such that $\mathscr{D}\left(\mathscr{D}_{\alpha}(\mathscr{S})\right) \cong \mathscr{D}(\mathscr{W})$. If $\mathscr{D}_{\alpha}(\mathscr{S})$ contains a line, then by Proposition 7.3 there is a spanning pair $\beta$ in $\mathscr{D}\left(\mathscr{D}_{\alpha}(\mathscr{S})\right)$ such that $\mathscr{D}_{\beta}\left(\mathscr{D}\left(\mathscr{D}_{\alpha}(\mathscr{S})\right)\right) \cong \mathscr{D}_{\alpha}(\mathscr{S})$. Let $\varphi: \mathscr{D}\left(\mathscr{D}_{\alpha}(\mathscr{S})\right) \rightarrow \mathscr{D}(\mathscr{W})$ be an isomorphism. Then $\beta^{\varphi}$ is a spanning pair of $\mathscr{D}(\mathscr{W})$ and $\mathscr{D}_{\beta \varphi}(\mathscr{D}(\mathscr{W})) \cong \mathscr{D}_{\alpha}(\mathscr{S})$. If $\mathscr{D}_{\alpha}(\mathscr{S})$ is a singleton, then $\mathscr{D}\left(\mathscr{D}_{\alpha}(\mathscr{S})\right)=\{\emptyset\}$ and hence, $\mathscr{D}(\mathscr{W})$ is a singleton, too. Hence, $\mathscr{D}(\mathscr{D}(\mathscr{W}))=\{\mathscr{D}(\mathscr{W})\} \cong \mathscr{D}_{\alpha}(\mathscr{S})$.

\section{Final remarks}

We give an example to show that in non-degenerate polar spaces there might be two disjoint generators, which form no spanning pair and that a dual polar space might contain non-isomorphic ADPs. Furthermore, the two components of an ADP do not have to be isomorphic. In fact, in this example all "bad things" (for which we already found examples) happen.

Let $V:=\mathbb{Q}^{(\mathbb{N})}=\bigoplus_{i \in \mathbb{N}} \mathbb{Q}$ and let $B:=\left\{b_{i}:=\left(\delta_{i j}\right)_{j \in \mathbb{N}} \mid i \in \mathbb{N}\right\}$ be the canonical basis of the $\mathbb{Q}$-vector space $V\left(\delta_{i j}\right.$ denotes the Kronecker Symbol). With $\beta$ we denote the standard scalar product $V \times V \rightarrow \mathbb{Q}:\left(\sum_{i \in \mathbb{N}} \lambda_{i} b_{i}, \sum_{i \in \mathbb{N}} \kappa_{i} b_{i}\right) \mapsto \sum_{i \in \mathbb{N}} \lambda_{i} \kappa_{i}$. Let $V^{*}$ be the dual of $V$ and set $W:=V \times V \times V^{*}$. We define a quadratic form $q: W \rightarrow$ $\mathbb{Q}:(v, u, f) \mapsto(v+u)^{f}+\beta(v, u)$, where $v \in V, u \in V$ and $f \in V^{*}$.

Let $\mathscr{S}$ be the point-line space whose points are the singular 1-dimensional subspaces of $q$ and whose lines are the singular 2-dimensional subspaces of $q$. Then $\mathscr{S}$ is a nondegenerate dual polar space.

Set $M_{v}:=\{\langle(v, 0,0)\rangle \mid v \in V\}$ and $M_{u}:=\{\langle(0, u, 0)\rangle \mid u \in V\}$. Then $M_{v}$ and $M_{u}$ are disjoint generators. Let $f_{a} \in V^{*}$ be the linear form with $\left(\sum_{i \in \mathbb{N}} \lambda_{i} b_{i}\right)^{f_{a}}=\sum_{i \in \mathbb{N}} \lambda_{i}$ and set $H:=\left\langle\left(0,0, f_{a}\right)\right\rangle^{\perp} \cap M_{v}$. Then $H^{\perp} \cap M_{u}=\emptyset$ and hence, $p^{\perp} \neq H$ for every $p \in M_{u}$. Hence by Proposition 6.3 we conclude that $\left(M_{v}, M_{u}\right)$ is no spanning pair.

Set $M_{f}:=\left\{\langle(0,0, f)\rangle \mid f \in V^{*}\right\}$. Then $M_{f}$ is a generator of rank $|\mathbb{R}|$, whereas both $M_{v}$ and $M_{u}$ are of rank $|\mathbb{N}|$. Furthermore, $\left(M_{v}, M_{f}\right)$ and $\left(M_{u}, M_{f}\right)$ are both spanning pairs. Set $\mathscr{W}_{a}:=\mathscr{D}_{\left(M_{v}, M_{f}\right)}(\mathscr{S})$. Then every point in $M_{v} \mathscr{W}_{a}$ is contained in $|\mathbb{R}|$ different lines. Against this, every point in $M_{f} \mathscr{W}_{a}$ is contained in exactly $|\mathbb{N}|$ different lines.

Set $U_{0}:=\left\{\left\langle\left(b_{2 i}, 0,0\right)\right\rangle \mid i \in \mathbb{N}\right\}$ and $U_{1}:=\left\{\left\langle\left(b_{2 i+1}, 0,0\right)\right\rangle \mid i \in \mathbb{N}\right\}$. Then $\alpha:=$ $\left(U_{0} \oplus M_{f}, U_{1} \oplus M_{f}\right)$ is a spanning pair. The two connected components of $\mathscr{D}_{\alpha}(\mathscr{S})$ are isomorphic and all points of $\mathscr{D}_{\alpha}(\mathscr{S})$ are contained in $|\mathbb{R}|$ different lines. We conclude that the two ADPs $\mathscr{W}_{a}$ and $\mathscr{D}_{\alpha}(\mathscr{S})$ are non-isomorphic.

In the end we give a list of open problems which arose in conjunction with this article and could not be solved, yet.

Question 8.1. To obtain an ADP out of a polar space, this polar space needs to have a spanning pair. Hence one might ask if there is a polar space without any spanning pair. We cannot answer this question by now. We do not even know if there is a polar space that possesses a generator that is not part of a spanning pair. For polar spaces of finite 
rank, there is for every generator a generator such that the intersection equals the radical. Such two generators always build a spanning pair.

Question 8.2. By Proposition 4.2 the opposition relation for an ADP of finite diameter is uniquely determined up to an automorphism of the underlying point-line space. As a consequence, two irreducible LDPs that consist of two connected components of finite diameter are isomorphic if and only if their underlying point-line spaces are. This is because the two connected components are coparallel and therefore isomorphic via mapping the points of the one component onto their coprojections in the other one. Hence, choosing of each LDP one connected component and an isomorphism between them gives canonically rise to an isomorphism between the two LDPs.

Are there non-isomorphic ADPs of infinite diameter whose underlying point-line spaces are isomorphic? Are there even ADPs with isomorphic underlying point-line spaces that are not dual-isomorphic?

Question 8.3. By Theorem 4.9 and Proposition 5.22 a dual polar space is an ADP if and only if the underlying polar space has finite rank.

There are examples of polar spaces of infinite rank whose duals are not even irreducible: Set $\mathcal{P}:=\mathbb{Z} \backslash\{0\}$ and $\mathcal{L}:=\{\{u, v\} \subseteq \mathcal{P} \mid u+v \neq 0\}$. Then $(\mathcal{P}, \mathcal{L})$ is a non-degenerate polar space.

For a generator $G$ of $(\mathcal{P}, \mathcal{L})$ and a point $x$, we have either $x \in G$ or $-x \in G$. Hence $G^{\prime}:=\{-p \mid p \in G\}$ is the unique generator disjoint to $G$ and moreover, $\left(G, G^{\prime}\right)$ is a spanning pair. This implies that the spanning pairs induce an opposition relation for the dual polar space $\mathscr{S}$ of $(\mathcal{P}, \mathcal{L})$ and therefore, $\mathscr{S}$ is a local dual polar space.

Since for every point of $\mathscr{S}$ there is a unique opposite point, we know that all irreducible components of $\mathscr{S}$ are atomic dual polar spaces consisting of two connected components. Hence, $\mathscr{S}$ is reducible.

Is there a polar space of infinite rank with an irreducible dual polar space?

Acknowledgements. I would like to thank the referee for the helpful remarks and the very thorough and detailed report which helped improving this article a lot.

\section{References}

[1] N. Bourbaki, Elements of mathematics. Algebra, Part I: Chapters 1-3. Hermann, Paris 1974. MR0354207 (50 \#6689) Zbl 0319.17002

[2] F. Buekenhout, A. M. Cohen, Diagram Geometry. Springer, New York, to appear.

[3] F. Buekenhout, E. Shult, On the foundations of polar geometry. Geometriae Dedicata 3 (1974), 155-170. MR0350599 (50 \#3091) Zbl 0286.50004

[4] P. J. Cameron, Dual polar spaces. Geom. Dedicata 12 (1982), 75-85. MR645040 (83g:51014) Zbl 0473.51002

[5] A. M. Cohen, Point-line spaces related to buildings. In: Handbook of incidence geometry, 647737, North-Holland 1995. MR1360727 (96k:51009) Zbl 0829.51004 
[6] P. M. Johnson, Polar spaces of arbitrary rank. Geom. Dedicata 35 (1990), 229-250. MR1066567 (91i:51006) Zbl 0755.51005

[7] J. Tits, Buildings of spherical type and finite BN-pairs. Springer 1974. MR0470099 (57 \#9866) Zbl 0295.20047

Received 14 May, 2009; revised 2 November, 2009

S. Huggenberger, Ghent University, Department of Pure Mathematics and Computer Algebra, Krijgslaan 281 - S22, 9000 Gent, Belgium

Email: simon@cage.ugent.be 